
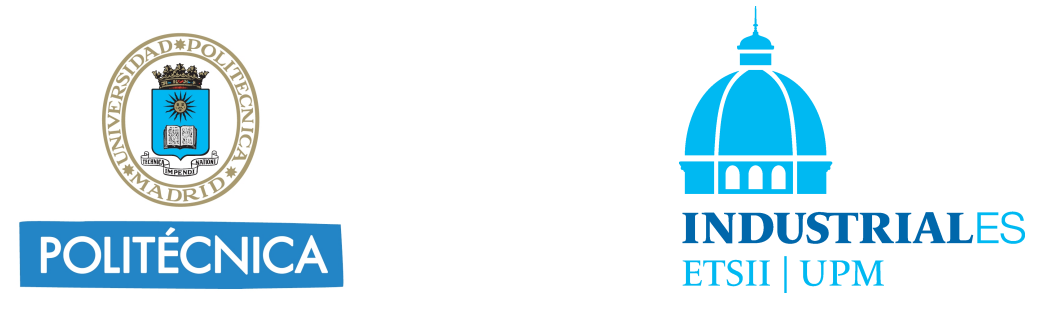

Universidad Politécnica de Madrid

Escuela Técnica Superior de Ingenieros Industriales

Doctorado Interuniversitario en Economía y Gestión de la Innovación (DEGIN)

Applications of artificial intelligence in behavioral finance getting benefit from extended data sources

\author{
A thesis submitted for the degree of \\ Doctor of Philosophy in Economy and Innovation \\ Management
}

\author{
Yang Liu \\ MBA
}

April, 2020 


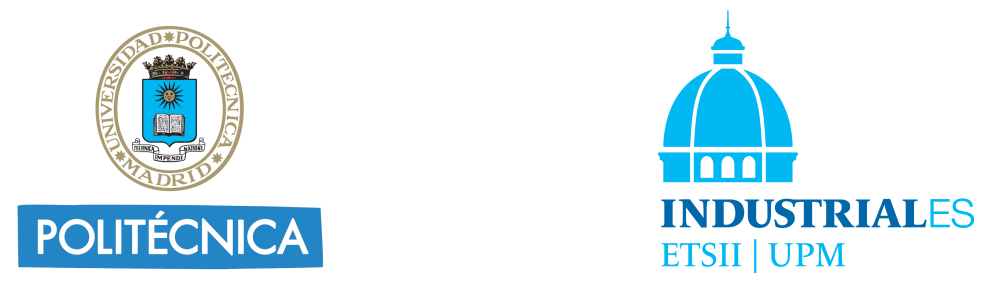

Escuela Técnica Superior de Ingenieros Industriales

Universidad Politécnica de Madrid

Applications of artificial intelligence in behavioral finance getting benefit from extended data sources

A thesis submitted for the degree of

Doctor of Philosophy in Economy and Innovation Management

\author{
Autor: \\ Yang Liu \\ MBA \\ Director: \\ Joaquín Ordieres Meré \\ Full professor
}

April, 2020 

Titulo:

\title{
applications of artificial intelligence in behavioral finance getting benefit from extended data sources
}

\author{
Autor: \\ Yang Liu \\ MBA
}

Tribunal nombrado por el Mgfco. y Excmo. Sr Rector de la Universidad Politécnica de Madrid el día..........de...... de 2020

\section{Tribunal}

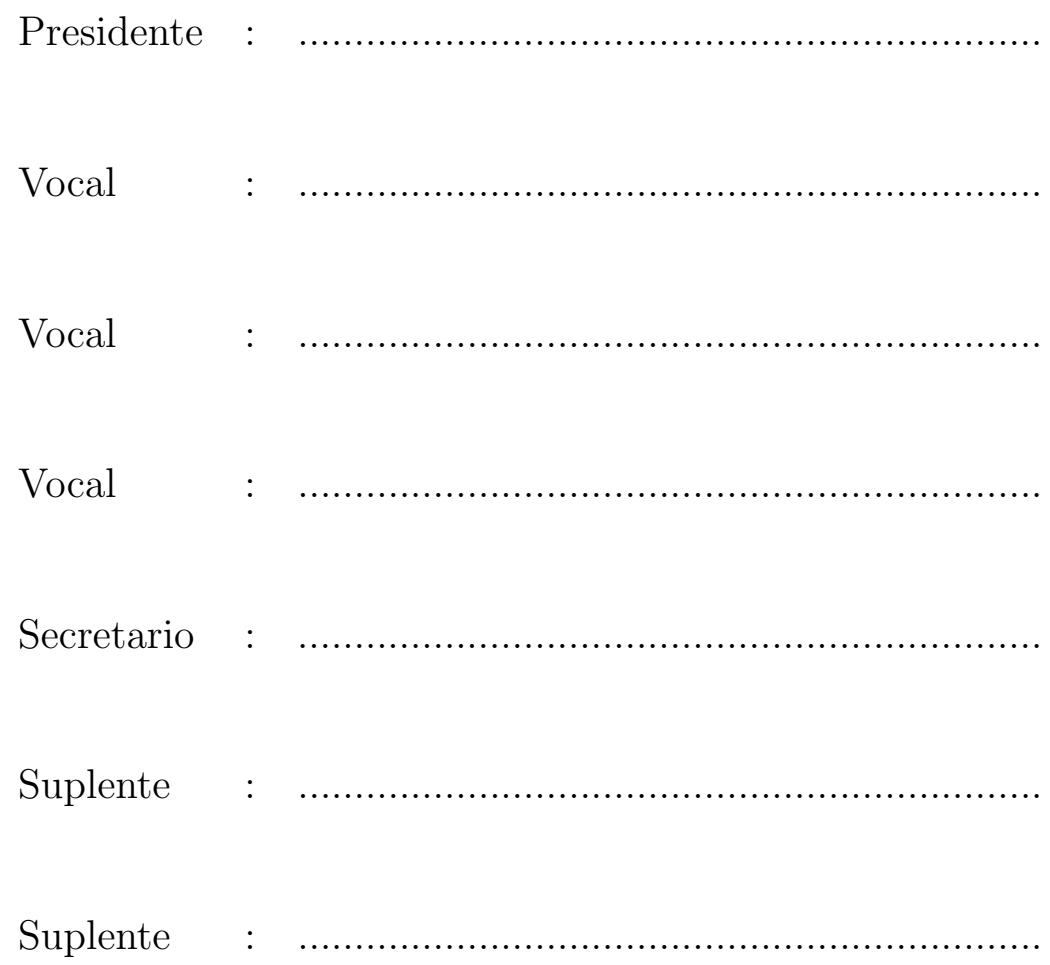

Realizado el acto de lectura y defensa de la tesis el día de de 2020 .

Calificación de la Tesis.......

El Presidente:

Los Vocales:

El Secretario: 



\section{Acknowledgements}

Back in time four years ago, that was my first meeting with Prof. Juaquín Ordieres Meré. Since then, the journey of my Ph.D. began with curiosity. This journey is full of painful and enjoyable experiences, whether in academic research or daily life. I am also grateful that I have received a lot of support, encouragement and help from my family and many friends. Without them, I could not complete my Ph.D. degree.

First, I would like to express my deep and sincere gratitude to my research supervisor, Prof. Juaquín Ordieres Meré for allowing me to do research and providing invaluable guidance throughout this research. His dynamism, vision, sincerity and motivation have deeply inspired me. He has taught me the methodology to carry out the study and to present the research works as clearly as possible. It was a great privilege and honor to work and study under his guidance. I am incredibly grateful for what he has offered me. I would also like to thank him for his friendship, empathy, and a great sense of humor. He is also the best example of a good professor, which will inspire my future career.

I am grateful to Prof. Donghong Ji for hosting me during my visit to Wuhan University. This is a beautiful experience that is an integral part of my $\mathrm{PhD}$ research. Meanwhile, I would also be grateful to Prof. Lili Ma for guiding me to research economics.

I am indebted to my friends and research colleagues, Adrián Carrió, Yanshen Liu, Hongyou Liu, Congke Yi, Xiaochen Zheng, Ermal Hetemi, Bobo Li, Hao Fei, Hao Tang, Qiji Zhou, Huanrui Yang, Qingguo Zeng, Wei Shi and Shizhu He. I want to give my special thanks to the professors, Prof. Francisco Javier Sanchez, Prof. Mercedes Rodríguez Paredes, Prof. Taiyuan Wang, Prof. Hang Dong, Prof. Hu Zhang, Prof. Meishan Zhang and Prof. Tao Qian.

I am extending my thanks to the universities, Xi'an International Studies University, Xi'an Jiaotong University, Universidad Complutense de Madrid, University of Cambridge, Wuhan University and Universidad Politécnica de Madrid, where provide me with the best environment for studying. Special gratitude goes to the China Scholarship Council (CSC) 
for offering me the scholarship making it possible for me to start my doctoral study at the very beginning.

Finally, I want to thank my parents for their understanding and selfless love. No matter what decision I make, they do their best to support me. Since I have been working and studying for almost ten years outside China, I am sorry that I cannot accompany or take care of them. I will make up for this regret for the rest of my time. They have always been the most important motivation for me to continue the journey of life. Moreover, thanks also to my future wife and children.

天资聪明鸣一时,

以勤补拙明一世。

Madrid

9.3.2020 


\section{Resumen}

Las finanzas conductuales son el núcleo de las finanzas modernas, que dependen en gran medida del valor de la datos información, que está en línea con las características del desarrollo tecnológico. En esta era de big data, la inteligencia artificial (IA) ha penetrado en todos los aspectos de nuestras vidas. Ha traído cambios revolucionarios a varios campos como las finanzas. Además, a medida que el desarrollo del machine learning en tecnología de IA y varias tecnologías de machine learning han utilizado ampliamente para realizar diferentes campos de las finanzas conductuales. Especialmente, deep learning ha demostrado un excelente desempeño en las tareas de finanzas conductuales. En general, esta investigación tiene como objetivo abordar los problemas en diferentes escenarios de aplicación de las finanzas conductuales con el apoyo de la tecnología de IA. La principal novedad de esta investigación construye un marco de aplicación probado, diferentes escenarios de las finanzas conductuales se benefician de la tecnología de IA.

Para examinar la viabilidad de los marcos propuestos, este estudio recoge datos del comportamiento humano de las redes sociales y las noticias financieras, que emplean tecnología de machine learning para analizar tres escenarios de aplicación en las finanzas conductuales. Este estudio extrajo con éxito la información del comportamiento humano a través del procesamiento del lenguaje natural (PLN), lo que ayuda a predecir el mercado de valores de la empresa. Mientras tanto, también exploró con éxito el impacto del contenido generado por el usuario (CGU) en el rendimiento de la empresa, lo que puede enriquecer la extracción de características para la evaluación del rendimiento de la empresa a través de las revisiones de los clientes. Los resultados experimentales de este estudio pueden ayudar a demostrar que la tecnología de IA es útil en escenarios de aplicación de finanzas conductuales y también promover en otros escenarios de aplicación similares. En resumen, este estudio proporciona una estrategia de marketing para los comercializadores de la empresa y decisiones comerciales para los gerentes de la empresa. 



\section{Abstract}

Behavioral finance is the core of modern finance, which relies heavily on the value of data information that is highly in line with the characteristics of technological development. In this era of big data, artificial intelligence (AI) has penetrated into every aspect of our lives. It has brought revolutionary changes to various fields such as finance. Furthermore, as the development of machine learning in AI technology and various machine learning technologies have been widely used to perform different the field of behavioral finance. Especially, deep learning has shown excellent performance in the tasks of behavioral finance. Overall, this research aims to address the problems in different application scenarios of behavioral finance with the support of AI technology. The main novelty of this research is to build a proven application framework, different behavioral financial scenarios benefit from AI technology.

To examine the feasibility of the proposed frameworks, this study collects human behavior data from social media and financial news, that employ machine learning technology to analyze three application scenarios in behavioral finance. This study successfully extracted human behavior information through natural language processing (NLP), which thereby helps to predict the stock market of the company. Meanwhile, it also successfully explored the impact of user-generated content (UGC) on company performance, which can enrich the feature extraction for company performance evaluation through customer reviews. The experimental results of this study can help to prove that AI technology is useful in application scenarios of behavioral finance and also promote in other similar application scenarios. In summary, This study provides a marketing strategy for the marketer of the company and business decisions for the managers of the company. 
ABSTRACT 


\section{Contents}

\begin{tabular}{|l|l}
\hline Acknowledgements & V
\end{tabular}

Resumen VII

\begin{tabular}{lll}
\hline Abstract & IX
\end{tabular}

\begin{tabular}{ll}
\hline List of Figures & XV
\end{tabular}

$\begin{array}{ll}\text { List of Tables } & \text { XVII }\end{array}$

\begin{tabular}{lll}
\hline 1 & Introduction & 1
\end{tabular}

1.1 Research motivation . . . . . . . . . . . . . . . . . . . . . . . . 1

1.2 Research objectives $\ldots \ldots \ldots \ldots \ldots$

1.3 Structure of the thesis dissertation $\ldots \ldots \ldots \ldots$

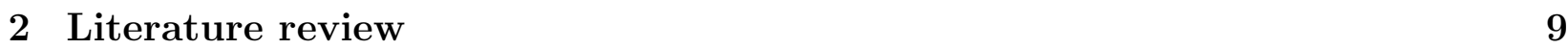

2.1 The impacts of UGC and eWOM on the company performance . . . . . . . . . 9

2.2 AI technology for company performance $\ldots \ldots \ldots \ldots \ldots$. . . . . . . . . 12

2.3 Bridge the gaps $\ldots \ldots \ldots \ldots \ldots \ldots$

2.4 Machine learning in AI technology for state of the art . . . . . . . . . . . . . 18

$2.4 .1 \quad$ Supervised learning . . . . . . . . . . . . . . . . . . . . . . . . . . . . . . 18

2.4 .2 Unsupervised learning . . . . . . . . . . . . . . . . . . . . 26

2.4 .3 Semi-supervised learning . . . . . . . . . . . . . . . 26

2.4 .4 Reinforcement learning . . . . . . . . . . . . . . . . . . . . . 27

2.5 Machine learning for natural language processing (NLP) $\ldots \ldots$. . . . . . . 27 
$2.5 .1 \quad$ Word embedding . . . . . . . . . . . . . . . . . . . 28

2.5 .2 Sentiment analysis $\ldots \ldots \ldots \ldots \ldots \ldots$

$2.5 .3 \quad$ Knowledge graph $\ldots \ldots \ldots \ldots$. . . . . . . . . . . . . . 29

2.6 Traditional statistical methods . . . . . . . . . . . . . . . . . . . . . 30

2.7 Performance measurement . . . . . . . . . . . . . . . . . . . . . 31

2.8 Tools and platforms $\ldots \ldots \ldots \ldots \ldots \ldots$

\begin{tabular}{|lll}
3 & Materials and frameworks design & 35
\end{tabular}

3.1 Data sources . . . . . . . . . . . . . . . . . . . . . . 37

$3.1 .1 \quad$ Application scenario 1 . . . . . . . . . . . . . . . . . . . . 37

$3.1 .2 \quad$ Application scenario 2 . . . . . . . . . . . . . . . . 41

$3.1 .3 \quad$ Application scenario $3 \ldots \ldots \ldots$. . . . . . . . . . . . . . . 48

3.2 Framework design $\ldots \ldots \ldots \ldots \ldots$. . . . . . . . . . . . . . . . . . 51

$3.2 .1 \quad$ Application scenario 1 . . . . . . . . . . . . . . . . 51

$3.2 .2 \quad$ Application scenario 2 $\ldots \ldots \ldots \ldots$

$3.2 .3 \quad$ Application scenario $3 \ldots \ldots \ldots$. . . . . . . . . . . . . . . 68

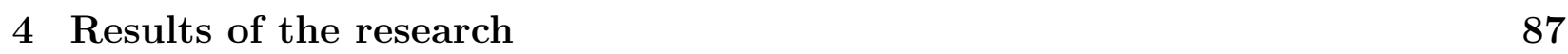

4.1 Application scenario $1 \ldots \ldots \ldots \ldots$. . . . . . . . . . . . . . . 87

4.1 .1 Thomson Reuters case study . . . . . . . . . . . . . . . . . 87

4.1 .2 Cable News Network case study . . . . . . . . . . . . . . . . . 90

4.2 Application scenario 2

4.2 .1 Company performance in the box office . . . . . . . . . . . . . . . 94

$4.2 .2 \quad$ Company performance in the stock market . . . . . . . . . . . . . . . . 101

4.3 Application scenario 3 . . . . . . . . . . . . . . . . . . . 106

4.3 .1 Case study in audience sentiment classification . . . . . . . . . . . . 106

4.3 .2 Case study in stock price movement . . . . . . . . . . . . . . . . . 108

\begin{tabular}{lll}
\hline 5 & Discussions and conclusions & 111
\end{tabular}

$5.1 \quad$ Discussions $\ldots \ldots \ldots \ldots \ldots \ldots \ldots$

5.1 .1 AI technology in this study . . . . . . . . . . . . . . . . 111 
$5.1 .2 \quad$ AI technology development by extended data sources . . . . . . . . . . 112

$5.1 .3 \quad$ Implications for business research . . . . . . . . . . . . . . . . . 114

5.1 .4 Limitations . . . . . . . . . . . . . . . . . . . . 115

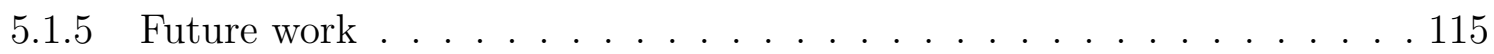

5.2 Conclusions . . . . . . . . . . . . . . . . . . . . . 116

5.2 .1 Contributions . . . . . . . . . . . . . . . . . . . . . . . . . 



\section{List of Figures}

1.1 Rising revenues worldwide of the financial sector in AI from 2016 to 2025. . . . 4

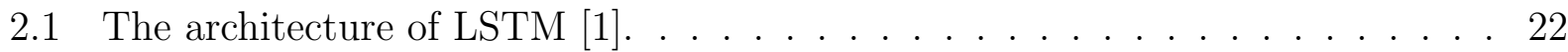

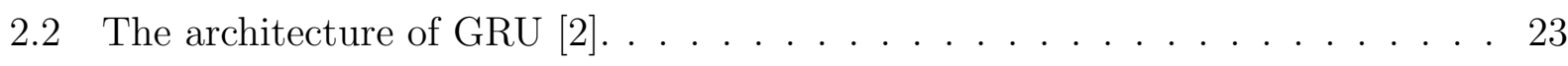

2.3 The architecture of attention mechanism model $[3]$. . . . . . . . . . . . . . . . 25

2.4 The pseudocode of TransE model $[4] . \ldots \ldots \ldots$

$3.1 \quad$ A framework of decision making for the application of machine learning. . . . 36

3.2 (Color online) an example of UGC on Facebook [5] . . . . . . . . . . . . . . 42

3.3 An example of Zootopia's IMDb page [6]. . . . . . . . . . . . . . . . . . . . . 42

3.4 The timing of data collection periods. . . . . . . . . . . . . . . . . 44

3.5 Convolutional neural network architecture proposed for feature selection. . . . 52

3.6 Text description of the entity. . . . . . . . . . . . . . . . . . . 54

3.7 Proposed architecture for the feature combination model. . . . . . . . . . . . . 55

3.8 The traditional machine learning workflow through TF-IDF feature extraction. 65

3.9 Illustrative pipeline for the decision making of studios from narrative materials. 67

3.10 A view of HTAN neural network. . . . . . . . . . . . . . . . . . . . . 69

3.11 A view of HCAN neural network. . . . . . . . . . . . . . . . 78

4.1 Average accuracy of prediction based on different feature sets for each company in Reuters. . . . . . . . . . . . . . . . . . . . . . . . . . . . 89

$4.2 \quad$ Average accuracy achieved on the basis of news from CNN and different feature set. . . . . . . . . . . . . . . . . . . . . 92

$4.3 \quad$ Average accuracy and F1-Score for each algorithm in the classification of audience's review through the rating-based method. . . . . . . . . . . . . . . . . 100 
List of Figures

4.4 Average accuracy and F1-Score for each algorithm in the prediction of stock price movement. . . . . . . . . . . . . . . . . . . . 103 


\section{List of Tables}

$2.1 \quad$ eWOM performance effects, the motion picture industry literature review, and the contributions of this research . . . . . . . . . . . . . . . . . . . 11

2.2 A review of financial expert system. ANN, NB, KNN, SVM, DT and SVR stand for artificial neural network, naive Bayes, k nearest neighbors, support vector machine, decision tree and support vector regression, respectively. Measure metrics: mean squared error (MSE); root mean square error (RMSE); area under the curve $(\mathrm{AUC}) . \ldots \ldots \ldots \ldots \ldots$

2.3 Confusion matrix. . . . . . . . . . . . . . . . . . . . . . . 31

3.1 Abstract of the research. . . . . . . . . . . . . . . . . . 35

3.2 Quotes and company names. . . . . . . . . . . . . . . . . 38

3.3 Samples extracted from the dataset. . . . . . . . . . . . . . . . . . . . 39

3.4 Training and text in the dataset. . . . . . . . . . . . . . . . . . . 39

3.5 Number of event characteristics labels for each company. . . . . . . . . . . . . 40

3.6 Definition of variables. . . . . . . . . . . . . . . . . . . . . . . 43

3.7 Quotes and studio names. . . . . . . . . . . . . . . . . . . 44

3.8 Training data and testing data in UGC. $\ldots \ldots \ldots \ldots$

3.9 Summary statistics of structured data in UGC. . . . . . . . . . . . . . . 47

3.10 The differences of models are used traditional machine learning and deep learning. . . . . . . . . . . . . . . . . . . . . . . . . 48

3.11 Information of review corpus in two cases of studios' performance. . . . . . . . 49

3.12 Time interval in training data and testing data . . . . . . . . . . . . . . . . 49

3.13 Features of the prediction model. . . . . . . . . . . . . . . . 51 
3.14 List of machine learning methods. . . . . . . . . . . . . . . . . . . . 63

3.15 Abbreviations of the results for each algorithm considered. . . . . . . . . . . . 64

3.16 Prediction model in classical machine learning. . . . . . . . . . . . . . . . 66

3.17 The differences of models are used traditional machine learning and deep learning. . . . . . . . . . . . . . . . . . . . . 85

4.1 Case study in the three application scenarios. . . . . . . . . . . . . . 87

4.2 Results for data extracted from Thomson Reuters. . . . . . . . . . . . . . . . . 88

4.3 Results for data extracted from Cable News Network. . . . . . . . . . . . . . . 91

4.4 The coefficient between UGC and box office (a). . . . . . . . . . . . . . . . . . 95

4.5 The coefficient between UGC and box office (b). . . . . . . . . . . . . . . 96

4.6 The coefficient between UGC and box office (c). . . . . . . . . . . . . . . 97

4.7 The accuracy and F1-Score of classification of the audience's review. . . . . . . 99

4.8 The coefficient between UGC and stock market (a). . . . . . . . . . . . . . . 102

4.9 The coefficient between UGC and stock market (b). . . . . . . . . . . . . . . . 104

4.10 The coefficient between UGC and box office (c). . . . . . . . . . . . . . . 105

4.11 The prediction accuracy and F1-Score of stock price movement. . . . . . . . . 105

4.12 Holistic comparison of traditional machine learning and deep learning for audience sentiment classification, the performance is measured based on accuracy, these values are a percentage i.e., the higher the better. . . . . . . . . . . . . 107

4.13 Holistic comparison of traditional machine learning and deep learning for stock price movement, the performance is measured based on accuracy, these values are a percentage. i.e., the higher the better. . . . . . . . . . . . . . . . . 108 


\section{1}

\section{Introduction}

\subsection{Research motivation}

Finance is an ancient industry. As early as $600 \mathrm{BC}$, there was a record of currency preservation and interest-bearing lending behaviors in Eurasia [7]. The rudimentary stage of the banking industry went back to the Venetian bank, which is in the Renaissance for more than 400 years ago. Silver ticket appeared in China at the end of the Ming Dynasty [8]. Subsequently, the big business group represented by Huizhou merchants and Shanxi merchants emerged, which laid the foundation for the development of the financial market in China [9]. On 9th March 1776, Adam Smith in the UK put forward the concept of "economics" in The Wealth of Nations [10], and everyone tries to expect their goods to gain the most value. At this moment, the specific meaning of modern finance is defined [11].

In the 20th century, Harry Markowitz defined the criteria for a portfolio [12]. His purpose was to achieve a compelling portfolio, which looks to build the maximum expected return under the established risks. The economist Fama proposed that Efficient Markets Hypothesis (EMH) [13], he systematically summarized research of EMH and introduced a complete theoretical framework. EMH is also an essential foundation for the modern standard financial theory, such as capital asset pricing model, arbitrage pricing, interest rate, exchange and option pricing theory, etc. Prof. Richard Thaler combined Human behavioral research with finance to become the founder of behavioral economic 14, 15. Prof. David Isaac Laibson 
adopted economics to analyze the psychological activities of people' investment [16], and incorporate people who are not $100 \%$ selfish assumptions into finance research. As a result, Behavioral finance is the study of the influence of psychology on the behavior of investors or financial analysts [17]. It has gradually assimilated into the research field of traditional finance, which can be used as an independent discipline. This discipline applies the hypothetical results for the practice of financial markets.

Behavioral finance analyzes the impact of humans' psychological, sentiment and social behaviors on investment decisions [18. Its primary research method is based on the results of empirical research on psychology. Even if behavioral finance has some limitations, it has also made a useful supplement and extension for modern financial theory. The modern financial theory emerged in the 1950s, which is beginning by [12. Several basic theories are building modern finance. For instance, the general equilibrium theory [19], the capital asset pricing model [20], the MM theory [21], and EMH [13], option pricing theory [22] and arbitrage pricing theory 23 .

At present, finance is to blend with new technologies in every time, which thereby brought enormous benefits to people. After experienced the electrical age and the information age, the financial industry quickly embraced the Internet industry and formed the new area of Internet finance. However, obtain full benefit from the internet is always a problem that plagues the global financial industry [24]. Especially in the intelligent era, new technologies represented by AI, blockchain, big data, and cloud computing have created new possibilities to address finance applications [25].

In 1950, Alan Turing asked, "Can machines think?" [26]. Humans began to think about how to give own their thoughts to the machines. At the summer workshop in Dartmouth Summer Research Project on Artificial Intelligence (AI), John McCarthy proposed that AI is about making the machines as smart as human behavior [27], AI began to be defined. People have begun to research and think about all aspects of AI. It is also hailed as the main driving force of the fourth industrial revolution and is disrupting almost every industry in the world [28].

AI is an emerging field of research, it aims to replicate and enhance human intelligence as intelligent machines through AI technology [29]. The research of AI has gone through three 
technological eras. In the first era, computers faced limitations in computing power. The second era emerged in the 1980s, as the further development of neural networks for machine learning, which enhanced computing capabilities for AI. Besides, Arthur Samuel coined the term "machine learning" in 1959. Machine learning is defined as a unique method of AI technology [30], which captures data and algorithms to apply them in new scenes and patterns without the need for direct programming. Driven by the development of deep learning, the current world is experiencing the third era of AI research, which builds more complex neural networks and applies real-life more effectively. The most significant difference between the third era and the previous technology is the lack of human intervention in the system. At that moment, At this time, deep learning has attracted people's attention, which is seen as a branch of machine learning [31]. It provides the necessary algorithms for the machine, which understands the basic principles of operation and essential parts of the data; it also deepens the knowledge and skills for machine learning [32].

Since AI technology has a stronger power to handle big data, finance has become one of the first industries to combine with AI [33]. AI technology has been the main driver in innovation capabilities [34]. Moreover, most of the significant technology companies are committed to AI research, such as Apple, Amazon, Tencent and Alibaba. 75\% technology companies believe that AI will open up new businesses while also providing competitors new ways to gain access to their markets. According to market research IDC [35], Figure 1.1 shows that finance has become the first industry to invest heavily in AI over the past three years.

Currently, finance research is through AI technology, which has a dynamic relationship between investors' behavior and the financial market. This relationship usually depends on different application frameworks [36]. The fusion of behavioral finance and AI technology represents an emerging field of research with numerous open research opportunities 37. Hence, this thesis explores different application scenarios of behavioral finance through AI technology.

Furthermore, since the increasing popularity, growth and convenience of using AI technology, the application scenario of behavioral finance is an exciting research field. Overall, 


\section{Rising revenues worldwide in artificial intelligence Revenue in USD bn}

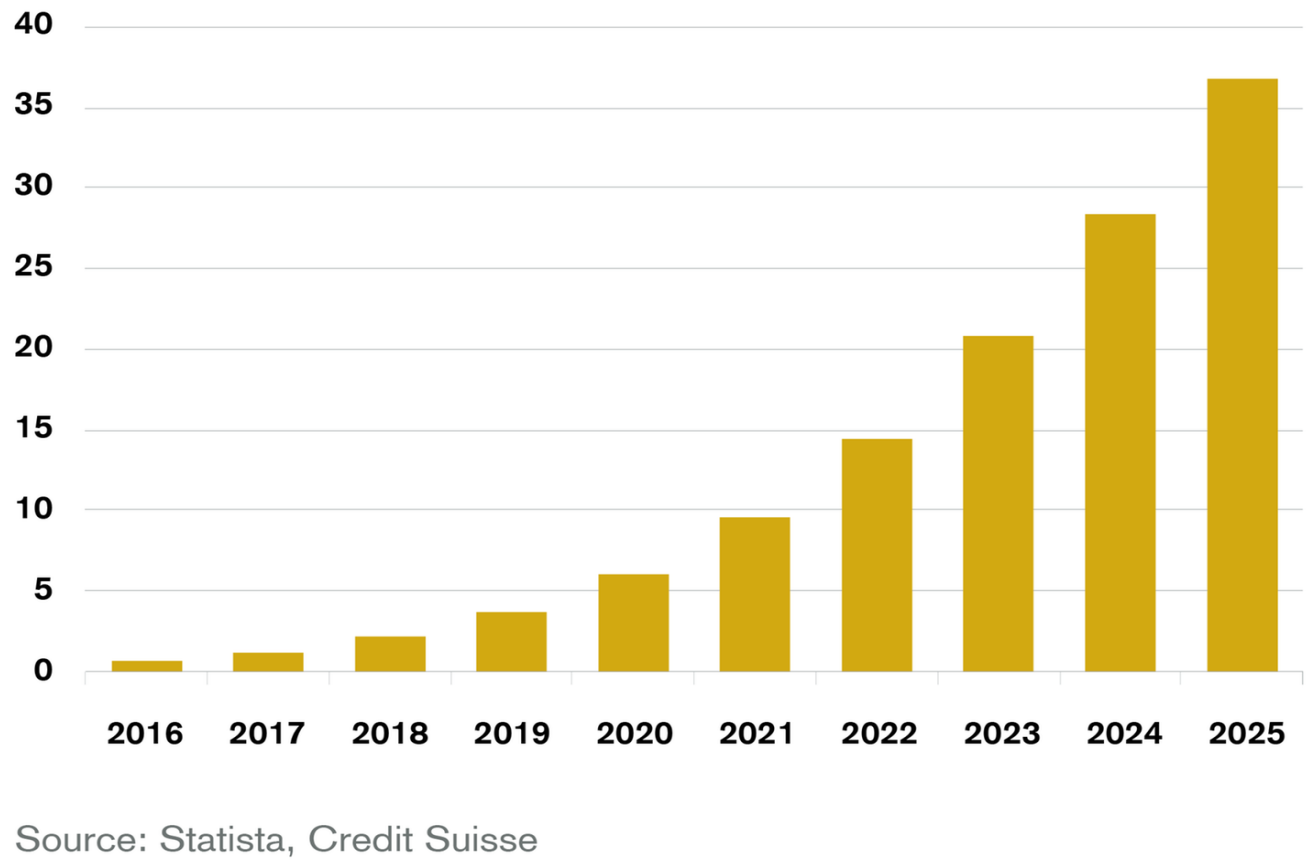

Figure 1.1: Rising revenues worldwide of the financial sector in AI from 2016 to 2025. 
this study proposes different technical frameworks for the three application scenarios which highlight the innovation of the structure in the application scenarios and discuss the various experimental results in detail. Three application scenarios in this thesis are proposed: 1). Anticipating stock market of the company [38, 39]; 2). Studio performance evaluation by a combined method; 3) Studio performance evaluation by an attention-based model [40]. Moreover, studio performance contains box office and stock market of studios.

\section{$1.2 \quad$ Research objectives}

The prediction of financial asset returns is one of the most frequently discussed topics in behavioral finance [41. Generally, investors are difficulty predicting stock market of company [42 45] considered whether the stock price movement would increase or decrease, to speculate on potential return and understand the factors of the development of financial markets. To modeling financial markets, people must first understand which variables influence price trends. The study [46] showed that the average forecast accuracy of the stock does not exceed $60 \%$ through the statistic model. And previous literature [47 49] showed that AI technology has significantly improvement for stock market predictions. Thus, this study could adopt AI technology to address prediction problems in financial markets. It aims to improve the prediction accuracy of stocks with advanced technology.

Moreover, many scholars have researched behavioral finance from the perspective of human behavior, including investor behavior and customer behavior [17]. These scholars mainly analyzed customer behavior affect the company's performance [50,51], which can provide business decisions for the managers of the company and increases the company's revenue. The new development of human behavior is powered by internet technology (Web 3.0), which is an emerging network information creation and a new resource organization model [52]. This new development generates user-generated content (UGC) that broadly refers to created text, pictures, audio and video published on the internet [53]. UGC also increases the emotional transmission of customers, which directly affects electronic word-of-mouth (eWOM) on company performance [54]. In summary, the impact of UGC and eWOM are essential factors for company performance. 
As the continuous change and evolution of the era of big data, more and more unstructured data will bring more opportunities and challenges to the research on company performance [55]. Existing literature also did not consider both structured and unstructured data that impact company performance [56], and these data are the main manifestations of human behavior. Obviously, the impact of human behavior on company performance is particularly important with AI technology [57].

Overall, this research utilizes AI technology for different application scenarios of behavioral finance. The objective of this thesis covers three aspects, which are depicted underneath:

- Objective 1: To better utilize human behavioral information to predict stock market of companies through AI technology.

- Objective 2: To better understand the impacts of user-generated content (UGC) on company performance evaluation.

- Objective 3: To provide helpful understanding behavioral finance applications based on AI technology.

\subsection{Structure of the thesis dissertation}

This doctoral thesis is divided into five chapters. A brief description of the remaining chapters follows:

- Chapter 2. Literature review. The second chapter introduce the application scenarios and the latest literature on $\mathrm{AI}$ in behavioral finance.

- Chapter 3. Materials and methods. This chapter provides a view of the data resource and methods, which consists of data descriptions for different application scenarios. It proposes a framework based on three application scenarios. This chapter also describes three framework processes and innovation points and sets the parameters for the three experiments. 
- Chapter 4. Results of the research. This chapter is devoted to describing and discussing the results of three application scenarios. This chapter also discusses the implications for business research, implications for practice and limitations.

- Chapter 5. Conclusions and future work. The last chapter summarizes the contributions and overall achievements of the three application scenarios in this thesis. Furthermore, this chapter proposes the direction of future work, which consists of ongoing and upcoming research activities. 



\section{2}

\section{Literature review}

\subsection{The impacts of UGC and eWOM on the company performance}

Most of the researchers often study UGC to understand how the information disseminated can help social media promotion and improve the movie market; it illustrates the crucial role of UGC in audience purchase decisions in the cinema. UGC further demonstrates the impact of audience behavior decisions on purchasing decisions [58] and product sales [59]. After the audience purchases, a continuous UGC is produced, which describes the audience's bandwagon effect [60]. The publishing advertising of the company in social media aims to advertise movie information to the audience [61]. It would attract more audience attention, which increases box office revenue and boosts the growth of the company stocks 62. Colicev et al. 63 proved that the UGC characteristic has a large number of publications with data sources that are extensive and direct, which affect the audience's sentiment. UGC reflects the opinions and mood of the audience in social media. Previous works pay attention to the audience's sentiment, which is an essential factor in the movie box office and stock market of the company [49]. Therefore, this study combines box office and stock market in company performance, which is the first to the analysis of the impact of UGC on company performance.

In general, the text of UGC format by the audience appears on IMDb, which fits well with 
data requirements in machine learning. Rutz et al. [64 utilized the bag of word approach to reveal text covariates and the latent dirichlet algorithm, which showed that high-order structures could predict advertisement marketing at different times. Rutz and Bucklin 65] evaluated the impact of semantics on paid search text advertisement through computational linguistics; it revealed that the brand search terms increased website visits and, thereby, affected brand marketing. Timoshenko and Hauser [66] developed a machine learning hybrid approach to identify customer needs from UGC, which provided a comparable set of customer needs from the experiential interviews and made professional marketing more efficient. UGC reflects the emotional orientation and demand characteristics of the audience. The incorporation of the UGC into the structured data from UGC is the best option for constructing a combined approach, and the best emotional performance stimulates the audience's enthusiasm for watching movies, encouraging the audience to buy the stocks of the company [62]. The above literature only considers unstructured data in the UGC, which ignores the impact of structured data on company performance. Thus, this article comprehensively analyzes structured and unstructured data in UGC on company performance.

Table 2.1 reviews publications that highlight eWOM performance effects on UGC. Prior to the movies was released, the companies utilized a survey of eWOM, which detects the sentiment performance of the audience, to reduce economic risks. Xun and Guo [78] explored whether positive and negative aspects of eWOM would impact target companies. The sensitivity ratio of eWOM is bigger, and users are more optimistic about the companies: they expect that the companies have higher returns and less volatility. However, the above works do not include any variables that capture the stock market of the company, this work can fill this gap in company performance.

Scholars measured the impact of eWOM through the parameters on UGC [79]. They also recognized the audience sentiment aspect of eWOM that may have a greater effect on the customer purchase decision. Audience sentiment is relatively easy to understand and can be either negative or positive, which becomes the metric in eWOM [80]. Kim et al. 81] examined the eWOM effects through consumer product reviews, which demonstrated the positive relationship between customer sentiment and attitudes towards company performance. 
Table 2.1: eWOM performance effects, the motion picture industry literature review, and the contributions of this research.

\begin{tabular}{|c|c|c|c|c|}
\hline Studies & $\begin{array}{l}\text { Theoretical } \\
\text { foundations }\end{array}$ & Method & $\begin{array}{c}\text { eWOM measure on } \\
\text { UGC }\end{array}$ & Case measure \\
\hline 67 & $\begin{array}{c}\text { Market } \\
\text { motivation }\end{array}$ & Regression & $\begin{array}{l}\text { Average rating, } \\
\text { number of ratings }\end{array}$ & Box office revenue \\
\hline 68 & Market signal & Regression & $\begin{array}{l}\text { Number of posts, } \\
\text { reviewer valence }\end{array}$ & Box office revenue \\
\hline 69 & $\begin{array}{l}\text { Retail feedback } \\
\text { signal }\end{array}$ & $\begin{array}{c}\text { Dynamic } \\
\text { simultaneous } \\
\text { equation system }\end{array}$ & Retail sales & Box office sales \\
\hline 70 & $\begin{array}{c}\text { Market } \\
\text { sentiment }\end{array}$ & Regression & Reviewer valence & Review helpfulness \\
\hline 71 & $\begin{array}{c}\text { Market } \\
\text { dialectics }\end{array}$ & $\begin{array}{l}\text { Two-sided } \\
\text { argument }\end{array}$ & $\begin{array}{c}\text { Reviewer's } \\
\text { arguments, number } \\
\text { of ratings }\end{array}$ & Review helpfulness \\
\hline $\mid 72$ & $\begin{array}{l}\text { Market } \\
\text { motivation }\end{array}$ & Regression & $\begin{array}{l}\text { Average rating, } \\
\text { reviewer valence }\end{array}$ & Box office revenue \\
\hline 73 & $\begin{array}{c}\text { Market } \\
\text { sentiment }\end{array}$ & Regression & $\begin{array}{l}\text { Review valence, } \\
\text { review quantity }\end{array}$ & Review helpfulness \\
\hline 74 & $\begin{array}{l}\text { Market } \\
\text { motivation }\end{array}$ & $\begin{array}{l}\text { Machine } \\
\text { learning }\end{array}$ & $\begin{array}{l}\text { Average rating, } \\
\text { reviewer valence }\end{array}$ & Box office revenue \\
\hline 75 & $\begin{array}{c}\text { Market } \\
\text { motivation }\end{array}$ & $\begin{array}{l}\text { Machine } \\
\text { learning }\end{array}$ & $\begin{array}{l}\text { Average rating, } \\
\text { reviewer valence }\end{array}$ & Box office sales \\
\hline 76 & $\begin{array}{c}\text { Market } \\
\text { sentiment }\end{array}$ & $\begin{array}{l}\text { Regression, } \\
\text { machine } \\
\text { learning }\end{array}$ & $\begin{array}{l}\text { Reviewer volume, } \\
\text { reviewer valence }\end{array}$ & Box office sales \\
\hline 77 & $\begin{array}{l}\text { Roger's } \\
\text { innovation } \\
\text { diffusion }\end{array}$ & Regression & $\begin{array}{l}\text { Type of review, } \\
\text { review volume }\end{array}$ & Box office revenue \\
\hline $\begin{array}{l}\text { This } \\
\text { study }\end{array}$ & $\begin{array}{l}\text { Sentiment- } \\
\text { finance } \\
\text { interface }\end{array}$ & $\begin{array}{l}\text { Machine } \\
\text { learning, deep } \\
\text { learning }\end{array}$ & $\begin{array}{l}\text { Average rating, } \\
\text { reviewer valence, } \\
\text { review quantity }\end{array}$ & $\begin{array}{l}\text { eWOM, stock price } \\
\text { movement }\end{array}$ \\
\hline
\end{tabular}


Moreover, Yin et al. 82] examined the relationship between sentiment and consumer, which proved that positive sentiment facilitates consumer product purchases. Previous works 83,84 further illustrated that consumer sentiment directly affects a company's stock returns. This study specifically investigated audience sentiment in the valence feature of eWOM, which fully taps the needs of the audience to determine precise marketing for the companies. This study proposes a way to go beyond the valence of eWOM on UGC and instead utilize the attention mechanism to improve the effect of eWOM on company performance.

\subsection{AI technology for company performance}

In Table 2.2 is evidence hoe the application of machine learning techniques to stock market prediction is an emerging research field. The advantage of machine learning models is their ability to facilitate the processing of large amounts of data. A common procedure to these models is the concatenation of features from different sources into a feature vector. For most machine learning models used, research is focused on predicting stock trends (increase or decrease). Section 2.5 introduces the meaning of the algorithm in machine learning and sentient analysis in natural language processing (NLP).

Various feature extractions have already been proposed to predict the stock price of the companies 47]. In early years, most of the stock market prediction depend on the empirical analysis of the econometric model. However, the previous work ignored the potential impact of unstructured data on company stocks. Since the bag-of-words is widely utilized in the task of document classification, the frequency of word appearance can be used as a feature of the classification 95 . Hence, the bags of words model only calculates the frequency of words and does not consider the word order or word sparsity in the context, which directly impacts the prediction result.

Behavioral finance had found that the sentiment of the investors often influences abnormal fluctuations in company stocks [41]. Tetlock [43] adopted the popular news from the Wall Street Journal to find that news sentiment has predictive power on company stocks. Chen et al. 96 found information co-mentions have a significant influence on the stock return through sentiment analysis. Furthermore, the investors' sentiment trends after the positive news that 
Table 2.2: A review of financial expert system. ANN, NB, KNN, SVM, DT and SVR stand for artificial neural network, naive Bayes, $\mathrm{k}$ nearest neighbors, support vector machine, decision tree and support vector regression, respectively. Measure metrics: mean squared error (MSE); root mean square error (RMSE); area under the curve (AUC).

\begin{tabular}{|c|c|c|c|c|}
\hline Paper & Text type & Feature extraction & Model type & $\begin{array}{c}\text { Performance } \\
\text { measure }\end{array}$ \\
\hline 85 & $\begin{array}{c}\text { Professional } \\
\text { newspaper }\end{array}$ & Stock data & NB & Accuracy \\
\hline 86 & $\begin{array}{c}\text { Professional } \\
\text { newspaper }\end{array}$ & Stock data & SVM & Accuracy \\
\hline 87 & Message board & Stock data & Regression & $\begin{array}{l}\text { Correlation } \\
\text { coefficient }\end{array}$ \\
\hline 88 & PRNewswire & Stock data & KNN, SVM & Accuracy, F1-score \\
\hline 89 & Aggregate news & $\begin{array}{l}\text { Stock data, } \\
\text { bag-of-words }\end{array}$ & SVR & MSE, accuracy \\
\hline 90 & $\begin{array}{l}\text { Corporate } \\
\text { disclosures }\end{array}$ & $\begin{array}{l}\text { Stock data, } \\
\text { bag-of-words }\end{array}$ & Multiple & $\begin{array}{l}\text { Accuracy, recall, } \\
\text { precision, F1-score }\end{array}$ \\
\hline 91 & Financial news & $\begin{array}{l}\text { Stock data, } \\
\text { sentiment }\end{array}$ & $\mathrm{DT}$ & $\begin{array}{l}\text { Accuracy, recall, } \\
\text { precision, F1-score }\end{array}$ \\
\hline 92 & Web media & $\begin{array}{l}\text { Stock data, } \\
\text { bag-of-words. }\end{array}$ & SVR & $\begin{array}{l}\text { Accuracy, recall, } \\
\text { precision, F1-score }\end{array}$ \\
\hline 93 & Mixed type & $\begin{array}{l}\text { Stock data, bag-of- } \\
\text { words, sentiment, }\end{array}$ & SVR & $\begin{array}{l}\text { RMSE, accuracy, } \\
\text { precision }\end{array}$ \\
\hline 46 & Message board & $\begin{array}{l}\text { Stock data, } \\
\text { sentiment }\end{array}$ & SVM & Accuracy \\
\hline 94 & Mixed type & $\begin{array}{c}\text { Stock data, } \\
\text { technical } \\
\text { indicators, } \\
\text { sentiment }\end{array}$ & $\begin{array}{c}\text { ANN, SVM, } \\
\text { DT }\end{array}$ & $\begin{array}{l}\text { Accuracy, AUC, } \\
\text { F1-score, precision }\end{array}$ \\
\hline 43 & Media news & Stock data & Regression & $\begin{array}{c}\text { Correlation } \\
\text { coefficient }\end{array}$ \\
\hline $\begin{array}{l}\text { This } \\
\text { research }\end{array}$ & Financial news & $\begin{array}{l}\text { Stock data, } \\
\text { technical } \\
\text { indicators, } \\
\text { bag-of-words, } \\
\text { syntax }\end{array}$ & Multiple & Accuracy, F1-score \\
\hline
\end{tabular}


results in a buying trend, and higher stock market prices while after negative news stocks are sold, resulting in a decrease in the price. Mittermayer and Knolmayer 88 illustrated that news-CATS achieve a performance that is superior to that of other ATC prototypes used to forecast stock price trends. Li et al. 93 proposed a media-aware quantitative trading strategy by using sentiment information on web media. The simulation trading return was up to $166.11 \%$. Nguyen et al. [46] offered a feature topic sentiment to improve the performance of stock market prediction. The above research fully illustrates the positive effect of sentiment analysis on stock price movement prediction.

This research utilizes the characteristics of syntax analysis as being proposed in [48, 97], namely, a structured tuple is extracted from an unstructured text based on the semantic structure of each piece of news. knowledge graph can enrich the structured representation of the news event and effectively retain feature vectors for the news event. The primary feature extraction in the previous studies $[98,99]$ are sentiment analysis, which neglected the event characteristics in the text. Furthermore, the existing literature [94, 99] had proved the positive effect of technical indicators on stock market prediction. In summary, this research highlights syntax analysis in financial news, which also incorporates other features extraction (stock data, technical indicators and bag-of-words). Because of the variety of considered features, this research will deliver an improved prediction of the stock market value for company in at least $3.6 \%$.

Previous research [47] was applied with the traditional machine learning algorithms. Especially, since the powerful application ability of deep learning based on machine learning. Deep learning is utilized in several studies for predicting stock price movement. Kraus and Feuerriegel [100] forecast the stock returns based on financial disclosures, and their result demonstrated that a higher directional of deep learning surpasses traditional machine learning. Ding et al. [101] illustrated that deep learning also can forecast stock market of the company. Chong et al. 102 examined three unsupervised feature extraction methods for stock market analysis prediction, and the results suggested that deep learning can improve prediction performance. The above literature proves that deep learning effectively improves the predictive performance of stock market.

The statistical models are the most commonly utilized in company performance in previ- 
ous literature [103. Banz 104 examined the empirical relationship between the return and the total market value of common stocks through ordinary least squares (OLS) regression in the movie market; he found that, on average, smaller companies had higher risk-adjusted returns than larger companies. Litman 105 pioneered the box office prediction; the feature selections such as story type, movie rating, star, cost and major distributors were considered independent variables, whereas the resulting economic rent was considered the dependent variable. This paper also used linear regression and achieved the author's expected results. Calantone et al. 106 also adopted OLS regression; however, the variables inputted were different. Tetlock [43] created a method for estimating the unknown parameters in the linear regression model. These results illustrated that negative words in company-specific news stories robustly predicted slightly lower returns on the following trading day. It is important to note that the regression analysis is limited in some aspects. Thence, machine learning methods can be considered in structural data.

The company performance had introduced through machine learning methods [49]. Sharda and Delen [107] used the neural networks to predict the financial performance of a movie based on its box-office receipts in one of nine categories. In Korea, Kim et al. 108 proposed a novel approach to the box office forecasting of motion pictures using social network service data and machine learning-based algorithms and developed three sequential steps (before, a week after and two weeks after the release) for predicting the box office. Their experiment results made noticeable improvements to the forecasting accuracy of all the three models. Liu [109] used machine learning to investigate whether UGC had a different impact on the stock market of the companies. Traditional machine learning methods mainly depend on feature extraction in human subjective design, which primarily contributes to the best performance for classifiers or regression models. Du et al. 110 predicted box office revenue from microblogs through machine learning, which showed that the prediction result is more accurate. However, the main problem of this research was that it only utilized classical machine learning algorithms, which do not consider feature selection in both structured and unstructured data; this causes the loss of more data features and increases the prediction error in the classification task. This study thereby adopts a combined approach (statistic method and machine learning method), which deals with feature selection in both structured 
and unstructured data.

Even if the deep learning model has been applied to the company performance [111, the attention mechanism can be seen as a way to make deep learning better [112], which can accurately capture sentiment words in context. Wang et al. 113 accurately captured the important sentiment characteristics of a given target sentence based on the attention mechanism. Chen et al. 114 proposed an attention-based sentiment classification model, which effectively captures global user preferences and product characteristics. He et al. [115] utilized an attention-based model to combine the crucial information of sentences, obtained from document-level sentiment classification, to achieve improved performance of sentiment classification. Liu and Zhang [116] combined left and right context using the two long shortterm memory (LSTM) attention models, which induced an attention value of the whole sentence. Song et al. [117] proposed a sentiment dictionary embedding method by attention mechanism that better represents the semantic relationship of sentiment words than the existing word embedding technique.

In addition, using attention mechanism can also extend to the stock market. $\mathrm{Hu}$ et al. [118] designed a hybrid attention network that predicted stock trends according to the chronological order of the news; this framework can significantly improve annualized returns. Liu et al. [119] adopted a two-level attention mechanism to quantify the importance of words and sentences in given financial news. This work is consistent with the proposed model, which ignores the importance of character levels and cannot understand the fine-grained sentiment characteristic. Furthermore, previous literature 120,121 also demonstrated the importance of character-level attention in natural language processing (NLP) tasks. In summary, this work employed attention mechanisms for both audience sentiment and stock market based on hierarchical attention networks (HAN) 122. This study combines the characteristics of these tasks and datasets to deeply transform the HAN model. The first attention layer in the hierarchical two-layer attention network (HTAN) model encodes character level, which fully captures the important characters in the words, and its second attention layer encodes the word level in the whole words, which also captures the important words list in the UGC. Meanwhile, the hierarchical convoluted attention network (HCAN) model can provide the best results, due to replacing recurrent neural network (RNN) with convolutional neural 
network (CNN) based on the HAN model. An ablation experiment was performed on the model to prove the effectiveness of the attention.

\subsection{Bridge the gaps}

\section{Gap 1: Feature selection for stock market prediction.}

Existing studies utilize financial news to predict stock market of company, their feature selection is mainly stock data, technical indicators, and bag of word. However, few literature consider synta features from the perspective of financial news, especially the characteristics of event tuples. Overall, this study adopts multiple models for predicting stock price of the company, which proves the reliability of the proposed model as comparing different algorithms. This work applies deep learning incorporated with knowledge graph embedding for feature extraction, which examines the applicability of combined features methods in the company stock market.

\section{Gap 2: Structured and unstructured data for company performance.}

Most of the existing literature only utilize structured or unstructured data in UGC, which evaluates company performance. However, a few literatures consider the characteristics of both structured or unstructured data. This work proposes using a combined approach both statistic method and machine learning for company performance. This work also can help managers make informed marketing decisions and provide them with information on audience enthusiasm timing, that monitors the emotional needs of the audience in real-time; which can help managers grasp the interests of the audience accurately.

\section{Gap 3: Electronic word-of-mouth effects on studio performance.}

Existing literature shows the following gaps: First, the eWOM characteristics on audience reviews are only reflected on the word level or sentence level; Second, since each audience review consists of multiple characters, word and sentences. These works did not consider these the fine-grain eWOM characteristics in the audience review. In this 
work, we adopted the attention mechanism evaluate company performance from audience review, this work can provide an applicable framework for studio performance, and this framework also should benefit academics and industry practitioners who provide insightful decision support for the motion picture industry.

\subsection{Machine learning in AI technology for state of the $\operatorname{art}$}

Since the 1980s, machine learning as a way to achieve AI, that has attracted widespread interest in real life. Especially in recent decades, research in the field of machine learning has developed rapidly and it has become an important part of AI. Machine learning is an application of AI that provides systems the ability to automatically learn and improve from experience without being explicitly programmed [123].

Among the several algorithms of machine learning, people will first consider how the algorithms are learned. Algorithms are classified according to learning methods, and the most suitable algorithm is selected according to the input data, that can obtain the best results in algorithm modeling or algorithm selection. There are four learning ways: supervised learning, unsupervised learning, semi-supervised learning and reinforcement learning [124].

\subsubsection{Supervised learning}

Supervised learning learns a function from a given training data set, which can predict results based on this function when inputs new data [125]. The training set requires for supervised learning includes input and output; it also calls features and goals. Humans label the targets in the training set.

For instance, in predictive spam processing, supervised learning establishes a learning process that compares the prediction results with the actual results of training data, it continuously adjusts the prediction model until the prediction results reach an expected accuracy rate. 


\section{Traditional machine learning}

- Support vector machine (SVM). This algorithm constructs hyperplanes or hyperplane sets in high-dimensional or infinite-dimensional spaces [126]. Given a set of training instances, each training instance is marked as belonging to one or the other of the two categories, which makes it a non-probability Meta linear classifier. SVM represents instances as points in space, so that the mapping allows the cases of the individual categories to be separated by as wide an apparent interval as possible. Afterward, map new instances to the same space and predict which category they belong to based on which side of the range.

- Logistic regression (LR). This algorithm is a generalized linear model, which solve classification for two classes [127. So it has many similarities with multiple linear regression. Thees model forms are basically the same, and they both have $w x+b$, where $w$ and $b$ are the parameters. Multiple linear regression directly uses $w x+b$ as the dependent variable, which $w$ is and logistic regression uses the function $\mathrm{L}$ to map $w x+b$ to a hidden state $p, p=L(w x+b)$, and then it determines the dependent variable according to the size of $p$ and $1-p$. If $L$ is a logistic function, it is logistic regression.

- Naive Bayes (NB). This algorithm is based on Bayesian theory, which assumes that the predictor is independent 128. Each instance $x$ can be described by the conjunction of the attribute values, and the objective function $f(x)$ takes an appraisal from a finite set $V$. The learner is provided with a series of training examples on the objective function, as well as new instances $\left\{a_{1}, a_{2} \ldots, a_{n}\right\}$, and then is required to predict the target value (or classification) for the new instance.

- Decision tree (DT). The goal of this algorithm is to create a model that predicts the value of a target variable based on several input variables [129. It uses a decision tree to go from observations about an item to conclusions about the item's target value. Tree models where the target variable can take a discrete set of values are called classification trees; in these tree structures, leaves represent class labels and branches 
represent conjunctions of features that lead to those class labels.

- Random forest (RF). This algorithm operates by constructing a large number of decision trees during training and outputting classes as classifications or regression. By the introduction of Leo Breiman [130], there are many decision trees in the forest and no correlation between each decision tree in the random forest. When a new input samples, each decision tree makes a judgment to see which category this sample for classification, and then one category is selected the most, and the sample is predicted to the type.

- AdaBoost (AB). This algorithm proposed by Yoav Freund and Robert Schapire [131]. It is an iterative algorithm that adds a new weak classifier in each round until it reaches a certain predetermined sufficiently small error rate. Each training sample is given a weight, indicating the probability that is selected into the training set by a classifier. If a sample point has been accurately classified, then the probability of being selected is reduced in constructing the next training set; on the contrary, if a sample point is not accurately categorized, its weight is increased.

- Gradient Boosting (GB). This algorithm produces a prediction model in the form of an ensemble of weak prediction models [132]. It builds the model in a stage-wise fashion as other boosting methods do, and it generalizes them by allowing optimization of an arbitrary differentiable loss function. The larger the loss function illustrates more error-prone in this model [133].

- Extreme Gradient Boosting (XGB). This algorithm is a gradient boosting algorithm and residual decision tree [134]. When each CRAT decision tree is added, the objective function is somewhat different has improved. Use multiple decision trees (multiple single weak classifiers) to form a combined classifier, and assign a certain weight to each nod. Compared to Gradient Boosting, XGB is fast computation speed and excellent model performance. 


\section{Deep learning}

Deep learning is a type of machine learning, which is the necessary path to achieve AI technology. Deep learning research on artificial neural networks, he contains multiple hidden layers of the perceptron [31]. Deep learning combines low-level feature representations with more abstract high-level representation attributes, which discovers distributed feature representations of data [32]. This algorithm is to build a neural network that simulates the human brain for analysis and learning. It mimics the mechanism of the human brain to interpret data, such as images [135], sounds [136 and text 137, 138, it also suitable for finance [139]. Currently, there are two major kinds of neural network architecture, that are convolutional neural network [140] and recurrent neural network [141].

\section{- Convolutional Neural Network (CNN)}

CNN is a specialized structure of multi-layer perceptron (MLP) [142]. This algorithm is a type of feedforward neural networks with a deep structure that includes convolution calculations, which is one of the representative algorithms of deep learning. CNN is composed of four parts: input layer, hidden layer, output layer. Compared with other deep learning structures [140]. CNN also can give better results in terms of image recognition [143].

In the hidden layer, it consists of a convolutional layer and a pooling layer. The purpose of the convolutional layer is to detect local collections from the previous layer, while the pooling layer can combine features with similar characteristics into one element. The cells in the convolutional layer are mapped in the feature map, where each unit is connected to the previous layer feature map by a set of weights. The result of the 1 ocally weighted sum will occur through the non-linear model. Then, the pooling layer would calculate the maximum local value in several features. The fully connected layer is located in the last part of the hidden layer of CNN. It only transmits signals to other fully connected layers, which is to combine the extracted features to get the output layer non-linearly.

\section{- Recurrent neural network (RNN)}




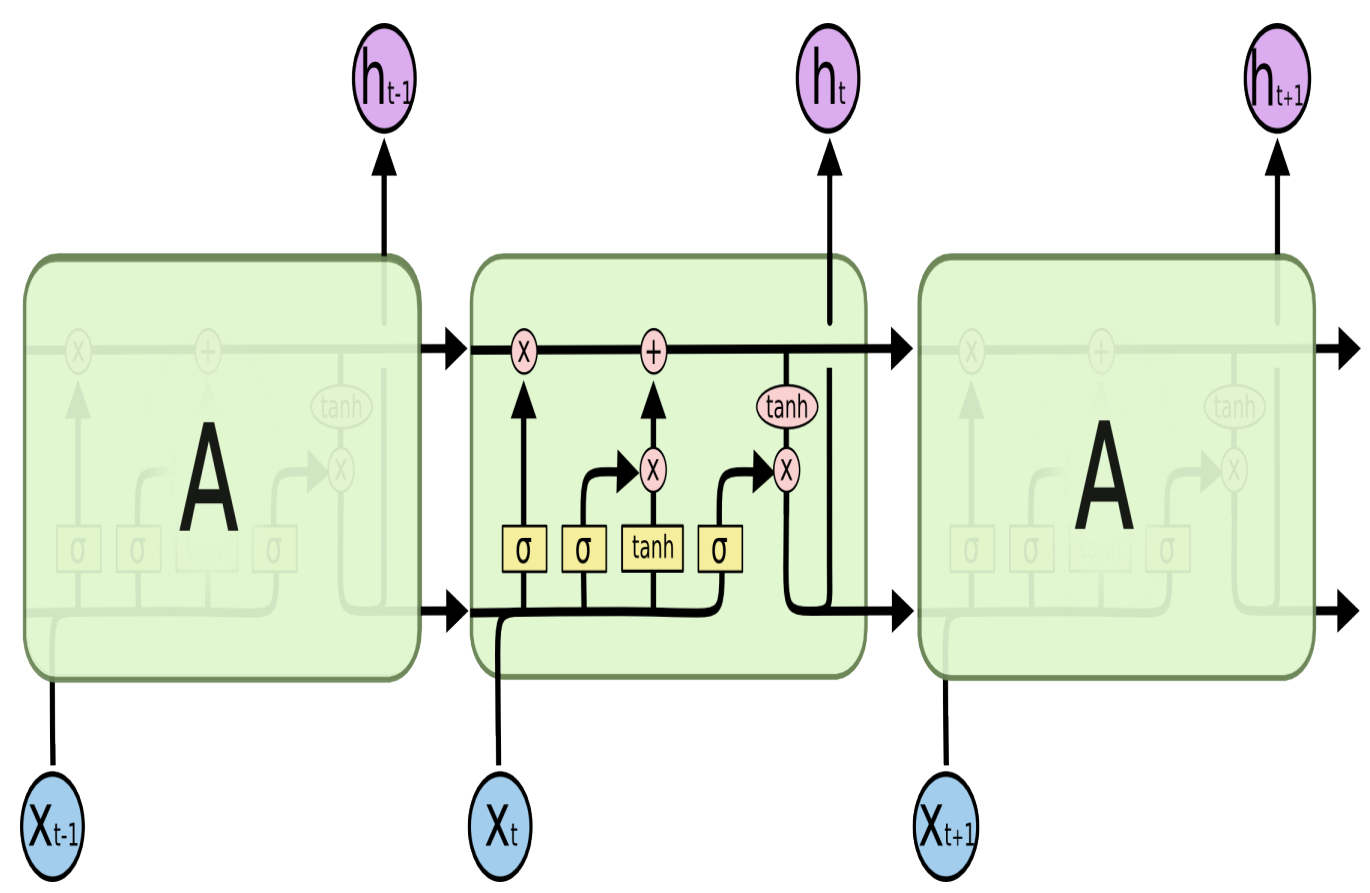

Figure 2.1: The architecture of LSTM 1].

Recurrent neural network (RNN) is a specialized structure for sequential data in the corpus; there are two variants in RNN that are long short-term memory (LSTM) and gated recurrent units (GRU). Different from $\mathrm{CNN}$, which is dedicated to processing features such as images, RNN is specifically designed to process feature sequences such as speech recognition [144] and language model [145].

LSTM [1] is a particular form of recurrent neural network; it is usually used to process sequence data and avoids the gradient explosion of traditional recurrent neural network or the problem of gradient disappearance [2]. LSTM captures long-distance dependencies in a sequence through the mechanism for memory cells and gates. In Figure 2.1, 


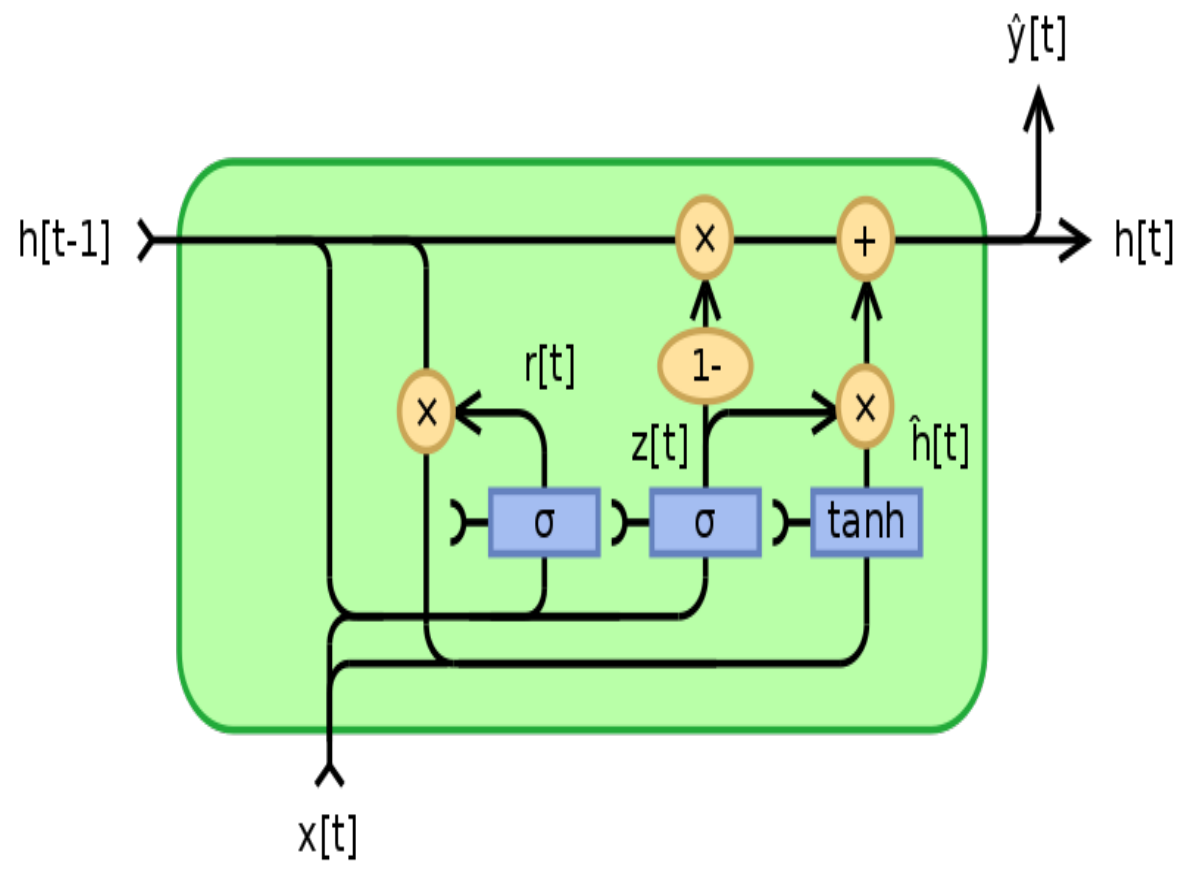

Figure 2.2: The architecture of GRU [2].

the calculation of LSTM is as follows:

$$
\begin{array}{r}
i_{t}=\sigma\left(W_{i} x_{t}+U_{i} h_{t-1}\right) \\
f_{t}=\sigma\left(W_{f} x_{t}+U_{f} h_{t-1}\right) \\
o_{t}=\sigma\left(W_{o} x_{t}+U_{o} h_{t-1}\right) \\
\widetilde{c_{t}}=\tanh \left(W_{c} x_{i}+U_{c} h_{t-1}\right) \\
c_{t}=f_{t} \odot c_{t-1}+i_{t} \odot \widetilde{c_{t}} \\
y_{t}=\tanh \left(o_{t} \odot c_{t}\right)
\end{array}
$$

where $\sigma$ represents the logistic sigmoid function, presents the operator of the point multiplication; $i_{t}, f_{t}, o_{t}$ and $c_{t}$ mean the input gate and forget gate, respectively. The output gate is at time, and activation vectors of the memory unit are the same dimension as the hidden layer vector $h_{t}$. The weight matrices $W$ and $U$ are the model parameters in LSTM.

GRU [2] has an excellent performance in sequence modeling. This algorithm is a variant 
of RNN that utilizes the gate mechanism to record the current state of the sequence. It can solve problems such as a gradient explosion. In Figure 2.2, the previous state $h_{t-1}$

and the current new state $\widetilde{h}_{t}$ information is computed by linear interpolation. Thus, the new state $h_{t}$ is computed by GRU as:

$$
h_{t}=\left(1-z_{t}\right) * h_{t-1}+z_{t} * \widetilde{h_{t}}
$$

The update gate $z_{t}$ is used to determine how much past information is retained and how much new information is added:

$$
z_{t}=\sigma\left(W_{z} d_{t}+U_{z} h_{t-1}+b_{z}\right)
$$

The $\mathrm{t}$ and the $d_{t}$ represent input word vectors, so the current update gate $h_{t}$ is represented as:

$$
\widetilde{h_{t}}=\tanh \left(W_{h} d_{t}+r_{t} *\left(U_{h} h_{t-1}+b_{n}\right)\right)
$$

The reset gate $r_{t}$ decides how much past information is applied to the candidate state. If $r_{t}$ is 0 , hence, the gate forgets all previous states. The $r_{t}$ is represented as:

$$
r_{t}=\sigma\left(W_{r} d_{t}+U_{r} h_{t-1}+b_{r}\right)
$$

\section{- Attention mechanism}

The attention model is improving the effects of the RNN-based with Encoder + Decoder [3], which is widely used in many fields, such as machine translation, speech recognition, and image recognition [146]. This method is firstly utilized in machine translation that assigns different weights to each word in a sentence. Meanwhile, attention itself also can explain the alignment relationship between translated input/output sentences [112]. In addition, the attention mechanism extracts more critical semantic information. This way would not bring more losses through the calculation and storage of the model. 


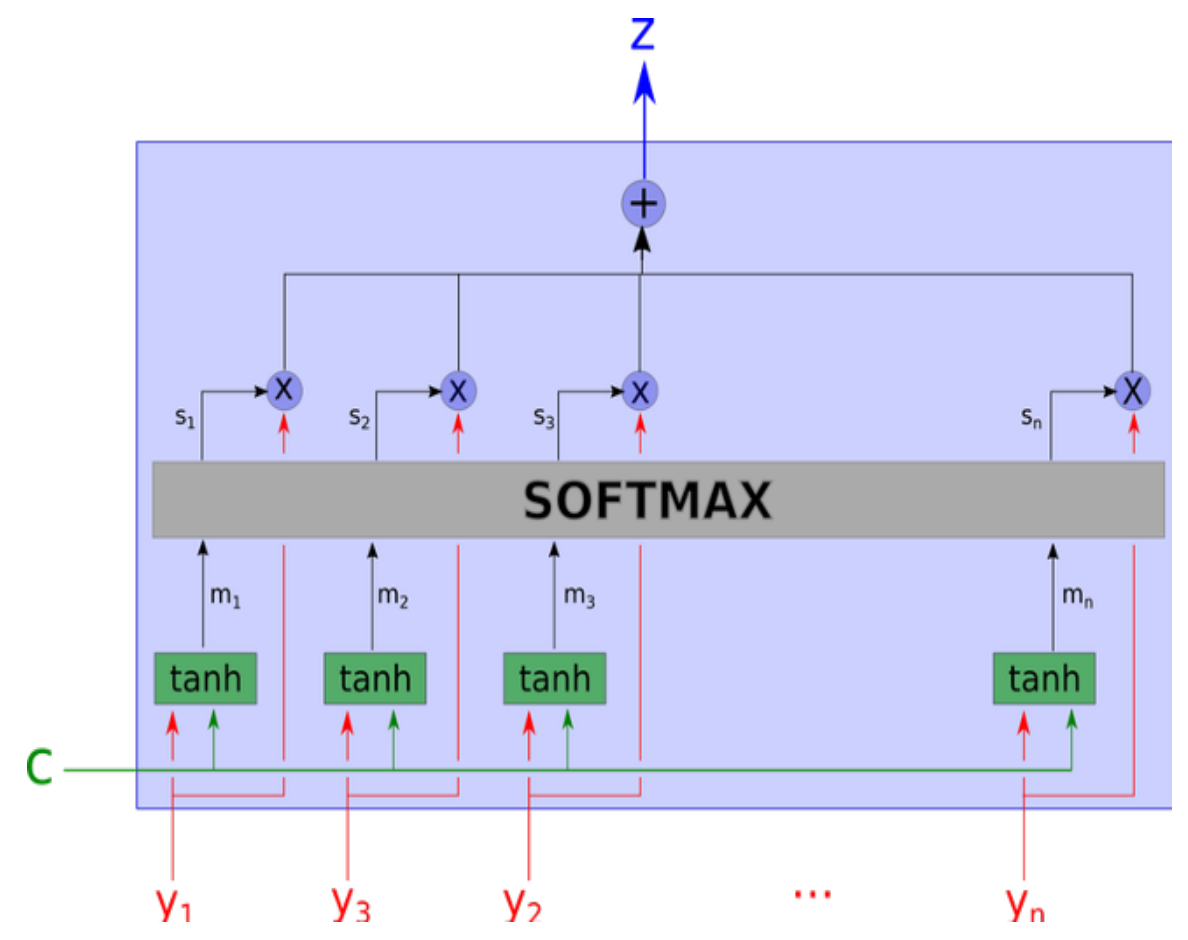

Figure 2.3: The architecture of attention mechanism model 3 .

Figure 2.3 tells us their workflow in attention mechanism. First, it defines the input $C$ as the context, and $y_{i}$ are word embedding in this corpus. Then it computes $m_{1}, \ldots m_{n}$ with tanh layer, this denotes an aggregation of $y_{i}$ and $C$ in the training process. Especially, each $m_{1}$ is an independent calculation that is without looking at $y_{j}, j \neq i$. This denotes as:

$$
m_{i}=\tanh \left(W_{c m} c+W_{y m} y_{i}\right)
$$

Second, each weight is computed by softmax, and $S=\operatorname{argmax}\left(m_{1}, \ldots m_{n}\right)=(0, \ldots, 0,1,0, \ldots, 0)$, where the only 1 in the output which input is the max. Then, the softmax is defined as:

$$
\operatorname{softmax}\left(x_{1}, \ldots, x_{n}\right)=\left(\frac{e^{x j}}{\sum_{j} e^{x j}} i\right)
$$


If one of $x_{i}$ is rather than any other number, $\operatorname{softmax}\left(x_{1}, \ldots, x_{n}\right)$ would be close to $\operatorname{argmax}\left(x_{1}, \ldots, x_{n}\right)$. Finally, it defines as:

$$
\begin{array}{r}
s \propto \exp \left(w_{m}, m_{i}\right) \\
\sum_{i} s_{i}=1
\end{array}
$$

where $s_{i}$ is softmax of $m_{i}$ projected on a direction, thus, the softmax can be considered as the max variable of the relevance based on the context.

$$
z=\sum_{i} s_{i} y_{i}
$$

Where output $z$ is the weight arithmetic mean of all the $y_{i}$, and the weight means the relevance for the context.

\subsubsection{Unsupervised learning}

Unsupervised learning is a type of machine learning that looks for previously undetected patterns in a data set with no pre-existing labels and with a minimum of human supervision [147]. Data acquisition consists of just a set of input data and looking for structures in the data, which can identify commonalities in the data. This method reacts based on the existence of such commonalities in each new data. Unsupervised learning algorithms mainly include principal components analysis (PCA) [148 and clustering [149].

\subsubsection{Semi-supervised learning}

Semi-supervised learning is an approach to machine learning that combines a small amount of labeled data with a large amount of unlabeled data during training. Semisupervised learning falls between unsupervised learning and supervised learning [150]. This learning method that combines supervised learning with unsupervised learning. It uses a 
large amount of unlabeled data and labeled data simultaneously for pattern recognition [151]. When used in conjunction with a small amount of labeled data, can produce considerable improvement in learning accuracy.

\subsubsection{Reinforcement learning}

This method emphasizes how to act on the environment to achieve the maximum expected benefits [152. Its inspiration comes from the behaviorist theory in psychology, that is, how the organism gradually forms the expectation of stimulation under the stimulation of the reward or punishment, which can produce the habitual behavior that can obtain the maximum benefits. Reinforcement learning performs better for problems involving long-term feedback than short-term feedback, which main applies to robot control and games 153.

\subsection{Machine learning for natural language processing $(\mathrm{NLP})$}

NLP mainly researches human language by automatic computation, which understands various theories and methods [154]. Belongs to an important research direction of AI, which is an interdisciplinary subject of computer science and linguistics. As the development of the Internet, especially, UGC text data has exploded, which has brought huge application requirements for NLP [155]. However, characteristics of NLP are ambiguous, dynamic and non-normative. And human language understanding usually requires rich knowledge and certain reasoning ability. Traditional machine learning provides a feasible solution to the above problems. Traditional machine learning has some problems in data acquisition and model construction. First, the network obtains large-scale labeled data. The conventional method will spend a lot of work on human labeling, which causes data sparseness. Second, it loses many features in manual extraction.

In recent years, the advancement of AI technology has provided solutions for the above aspects. Especially deep learning has effectively promoted the development of NLP [156]. 1). Using an average distribution vector to represent different language units, such as phrases, 
sentences, and chapters. 2). Using CNN and RNN combine different language units, which obtains a representation of a larger language unit and implements various NLP tasks through vector space operations [157]. For example, sentiment analysis, knowledge graph, etc.

\subsubsection{Word embedding}

Word embedding is the collective name for a set of language modeling and feature learning techniques in NLP [158], where words or phrases from the vocabulary are mapped to vectors of real numbers. Conceptually it involves a mathematical embedding from a space with many dimensions per word to a continuous vector space with a much lower dimension. This study utilizes word2vec [159] and glove [160] to train word embedding.

Word2vec is a group of related models that are used to produce word embedding [161. These models are shallow, two-layer neural networks that are trained to reconstruct linguistic contexts of words. Word2vec takes as its input a large corpus of text and produces a vector space, typically of several hundred dimensions, with each unique word in the corpus being assigned a corresponding vector in the space. It contains continuous bag-of-words (CBOW) and continuous skip-gram 162].

GloVe coined from Global Vectors, is a model for distributed word representation [160]. The model is an unsupervised learning algorithm for obtaining vector representations for words. This is achieved by mapping words into a meaningful space where the distance between words is related to semantic similarity.

\subsubsection{Sentiment analysis}

Sentiment analysis refers to the use of natural language processing, text analysis, computational linguistics, and biometrics to systematically identify, extract, quantify, and study affective states and subjective information [163. Sentiment analysis is widely applied to the voice of the customer materials, such as reviews and survey responses, online and social media, and healthcare 164.

A basic task in sentiment analysis is classifying the polarity of a given text at the document, sentence, or aspect level — whether the expressed opinion in a document, a sentence or 
an entity aspect is positive, negative, or neutral [165. From the perspective of subjectivity and objectivity identification, this task is commonly defined as classifying a given text into one of two classes: objective or subjective [163]. Meanwhile, from the perspective of aspectbased, it refers to determining the opinions or sentiments expressed on different features or aspects of entities [166].

Existing approaches to sentiment analysis can be grouped into three main categories: knowledge-based techniques, statistical methods and hybrid approaches [167]. Knowledgebased techniques classify text by affect categories based on the presence of unambiguous affect words such as happy, sad, afraid, and bored [168]. Statistical methods leverage elements from machine learning such as latent semantic analysis and bag of words for semantic orientation and machine learning [169]. Hybrid approaches leverage both machine learning and elements from knowledge representation such as ontologies and semantic networks in order to detect semantics that are expressed in a subtle manner [170].

\subsubsection{Knowledge graph}

Knowledge graph as a semantic network has reliable expressive power and modeling flexibility [171]. First, knowledge graph is a semantic representation that can model entities, concepts, attributes and their relationships in the real world; Secondly, knowledge graph is a data exchange standard for its derivative technology. Its technology is knowledge extraction, knowledge integration, knowledge management and knowledge application [172].

Knowledge graph embedding is a kind of representation learning in knowledge graph. Currently, several algorithms [172] explore the mapping relationship between entities and relationships in the translation distance model. The TransE model [4] is a computationally efficient predictive model that satisfactorily represents a one-to-one type of relationship. It has been used as a basis in the development of different translation distance models. The TransH model [173] maps the head and tail of a vector onto a hyperplane, after which these two components complete the translation process on the hyperplane. The TransR model [174], which is based on the TransE model, consists of entities and relationships that are located in different dimensions of space. For each relation- ship, the model defines a matrix that is used to convert entity vectors into space. The model is also dynamically determined by an 


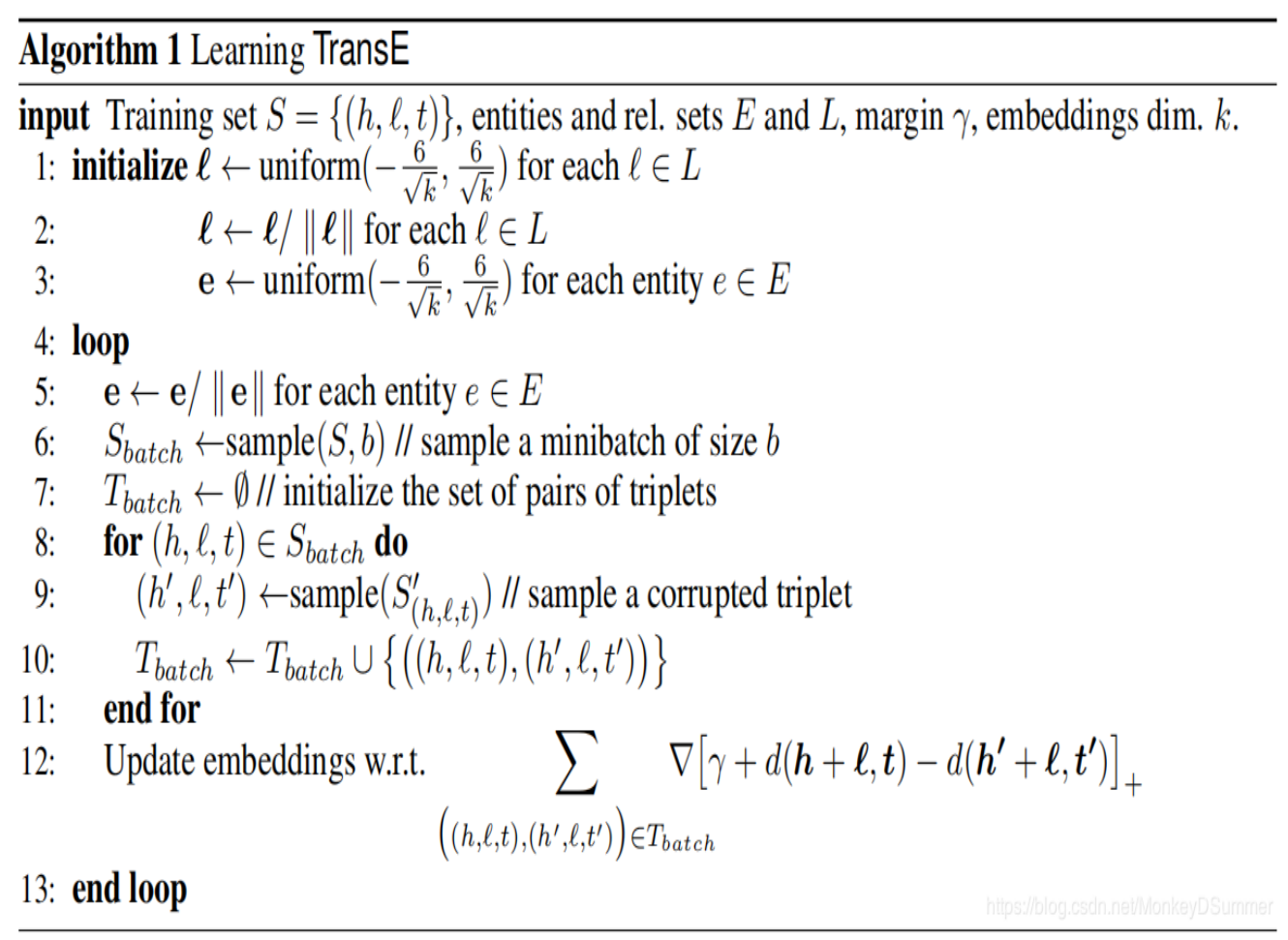

Figure 2.4: The pseudocode of TransE model [4].

entity relationship. The TransD model [175] is an improvement of the TransR model, which the former uses as a basis in considering a transformation matrix.

In this research, solving the head and tail of a news tuple for mapping in a single space necessitates the simple mapping of one-to-one entity extraction features. This functionality is found in the TransE model, its calculation process in Figure 2.4.

\subsection{Traditional statistical methods}

1) Multiple linear regression (MLR)

MLR is a predictive analysis, the multiple linear regression is used to explain the relationship between one continuous dependent variable and two or more independent variables 176 .

2) Ordinary least squares (OLS)

OLS is a type of linear least squares method for estimating the unknown parameters 
in a linear regression model [177]. OLS chooses the parameters of a linear function of a set of explanatory variables by the principle of least squares: minimizing the sum of the squares of the differences between the observed dependent variable in the given dataset and those predicted by the linear function. OLS is used in fields as diverse as econometrics and data science [178].

3) Panel data analysis

This methods is widely used in econometrics, which analyzes two-dimensional panel data $[179]$. The data are usually collected over time and over the same individuals and then a regression is run over these two dimensions. Multidimensional analysis is an econometric method in which data are collected over more than two dimensions.

\subsection{Performance measurement}

Many machine learning researches adopt accuracy, precision, recall and F1-score [180, which evaluates performance measurement in classification tasks. Table 2.3 shows confusion matrix in performance measurement, such as true positive(TP), false negative (FN), true negative (TN) and false positive (FP).

Table 2.3: Confusion matrix.

\begin{tabular}{ccc}
\hline & Positive & Negative \\
\hline True & TP & FP \\
False & FN & TN \\
\hline
\end{tabular}

- Accuracy is the percentage of the total sample that predicts the correct result.

$$
\text { Accuracy }=(T P+T N) /(T P+T N+F P+F N)
$$

- Precision is the probability of actually positive samples among all samples predicted to 
be positive.

$$
\text { Precision }=T P /(T P+F P)
$$

- Recall is the probability of being predicted as a positive sample in the actual positive sample.

$$
\text { Recall }=T P /(T P+F N)
$$

- F1-score is the harmonic mean of the precision and recall. The higher the F1-score, the more robust is in classification model.

$$
F 1-\text { score }=(2 * \text { Precision } * \text { Recall }) /(\text { Precision }+ \text { Recall })
$$

\subsection{Tools and platforms}

a) Traditional statistical methods

This study adopts Stata for panel data analysis. This software created in 1985 by StataCorp [181]. Most users work in research, especially in the fields of economics, sociology, political science, biomedicine and epidemiology. This software is a powerful tool and capable of panel data analysis, statistical analysis and regression analysis.

b) Machine learning

This study adopts Python with Scikit-learn for machine learning. Python is an objectoriented program 182]. Scikit-learn package is a free software machine learning library for the Python programming language [183]. It features various classification, regression and clustering algorithms.

c) Deep learning

This study adopts Python with Keras and TensorFlow and for deep learning. Keras is an open-source neural-network library written in Python [184]. Designed to enable fast 
experimentation with deep neural networks, it focuses on being user-friendly, modular and extensible.

TensorFlow is a free and open-source software library for data flow and differentiable programming across a range of tasks [185]. It is a symbolic math library, and is also used for machine learning applications such as neural networks. 



\section{3 \\ Chapter \\ Materials and frameworks design}

This section includes data sources, method and framework design. The chapter concludes with tools and platforms. In order to better understand the objectives of each study and the corresponding methods adopted, Table 3.1 summarizes three application scenarios, including application cases, data source and methods.

This study addresses three application scenarios of behavioral finance by machine learning. The data access supported by machine learning approaches can have a substantial impact on behavioral finance. This data process calls the decision support system [186], which can provide a business decision for the company and reduce the operation costs of the company.

Table 3.1: Abstract of the research.

\begin{tabular}{|c|c|c|c|}
\hline & Application scenario 1 & Application scenario 2 & Application scenario 3 \\
\hline Data source & $\begin{array}{c}\text { Financial news from } \\
\text { Cable News Network } \\
\text { and Thomson Reuters, } \\
\text { Stock data from Yahoo } \\
\text { finance }\end{array}$ & $\begin{array}{l}\text { Structured data from } \\
\text { Facebook (e.g. like, } \\
\text { share), unstructured } \\
\text { data (audience review } \\
\text { from IMDb) }\end{array}$ & $\begin{array}{c}\text { Audience review from } \\
\text { IMDb }\end{array}$ \\
\hline Methods & $\begin{array}{c}\text { Knowledge graph, } \\
\text { machine learning, deep } \\
\text { learning }\end{array}$ & $\begin{array}{l}\text { Statistics methods, } \\
\text { machine learning }\end{array}$ & $\begin{array}{c}\text { Machine learning, deep } \\
\text { learning, attention } \\
\text { mechanism }\end{array}$ \\
\hline $\begin{array}{l}\text { Framework } \\
\text { design }\end{array}$ & $\begin{array}{l}\text { Knowledge graph } \\
\text { approach }\end{array}$ & A combined approach & Attention-based model \\
\hline
\end{tabular}




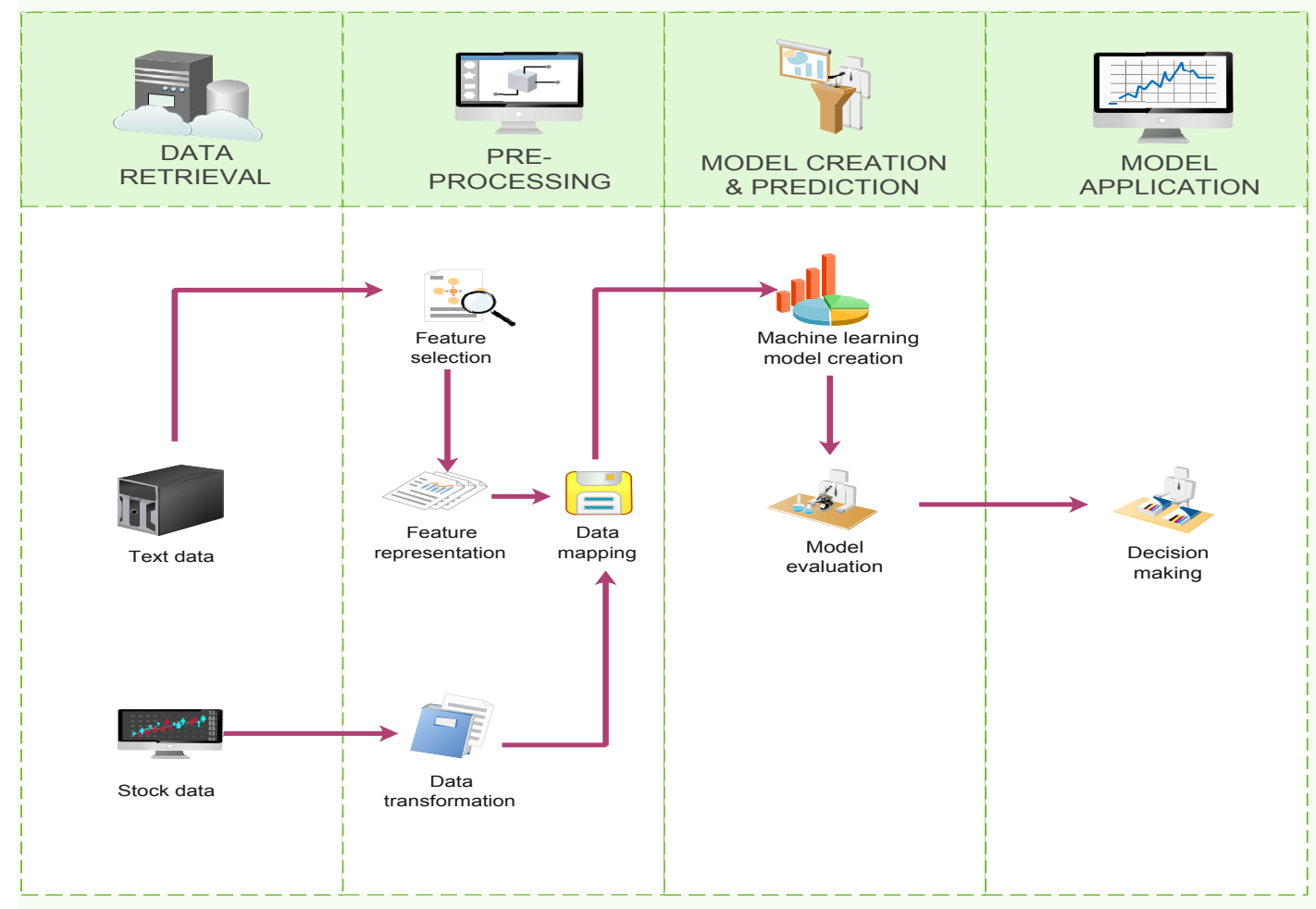

Figure 3.1: A framework of decision making for the application of machine learning. 
Hence, this study can select decision making method [187 to apply for three application scenarios, Decision making method is the process of making choices by identifying a decision, gathering information and assessing alternative resolutions. Decision making involves the selection of a course of action from among two or more possible alternatives in order to arrive at a solution for a given problem [188]. It effectively establishes a direct process to monitor the application scenarios and effectively influences the decision-making of the company.

A framework of decision making for the application of machine learning in behavioral finance is designed to guide the following research, as presented in Figure 3.1. This framework includes four steps: data retrieval, pre-possessing, model creation \& prediction and model application. The decision-making framework applies to the three application scenarios in this study. As mentioned in the previous sections, AI technology also has involved in many fields of behavioral finance, and it is possible to extend this framework to other areas. The advantage gained is that it can effectively predict financial activities and prevent financial risks, which provides business decisions to company managers promptly.

\subsection{Data sources}

\subsubsection{Application scenario 1}

\section{Dataset Description}

Table 3.2 shows the custom financial news corpus built with headlines from two datasets. The first dataset contains news articles published by Thomson Reuters, including those regarding Apple Inc. (AAPL), Microsoft Corporation (MSFT) and Samsung Electronics Co., Ltd. (SSNLF). The second dataset comprises news reports published in CNN, including reports on the Boeing Company (BA), Google Inc. (GOOG) and Walmart Inc. (WMT). It also consists of financial news headlines published at specific time intervals, with each news report accompanied by a title and a release date. Titles are used for event embedding and feature extraction, and release dates are used as a reference in ensuring alignment between corresponding financial news and trading data from a time series. As shown in previous work 100,101, using a headline to build corpora can help reduce noise in text mining as 
Table 3.2: Quotes and company names.

\begin{tabular}{|c|c|c|c|c|}
\hline $\begin{array}{c}\text { Financial news } \\
\text { dataset }\end{array}$ & Stocks & Company names & Time interval & $\begin{array}{c}\text { The number } \\
\text { of news } \\
\text { headline }\end{array}$ \\
\hline \multirow{3}{*}{$\begin{array}{c}\text { Thomson Reuters } \\
\text { (Reuters) }\end{array}$} & AAAL & Apple Inc. & $\begin{array}{l}3.10 .2011- \\
31.7 .2017\end{array}$ & 6423 \\
\hline & MSFT & $\begin{array}{l}\text { Microsoft } \\
\text { Corporation }\end{array}$ & $\begin{array}{l}30.9 .2011- \\
29.11 .2017\end{array}$ & 6623 \\
\hline & SSNLF & $\begin{array}{l}\text { Samsung } \\
\text { Electronics Co } \\
\text { Ltd. }\end{array}$ & $\begin{array}{l}30.9 .2011- \\
29.11 .2017\end{array}$ & 6900 \\
\hline \multirow{3}{*}{$\begin{array}{l}\text { Cable News } \\
\text { Network }\end{array}$} & $\mathrm{BA}$ & $\begin{array}{l}\text { The Boeing } \\
\text { Company }\end{array}$ & $\begin{array}{l}8.9 .1998- \\
23.2 .2017\end{array}$ & 2999 \\
\hline & GOOG & Google Inc. & $\begin{array}{l}25.5 .2006- \\
24.2 .2017\end{array}$ & 5278 \\
\hline & WMT & Walmart Inc. & $\begin{array}{l}7.5 .1997- \\
24.2 .2017\end{array}$ & 4913 \\
\hline
\end{tabular}

headlines concisely represent the content of a text. This also can effectively extract the characteristics from financial news.

Daily stock data from index report in each company are collected by Yahoo Finance in the same period during which stock data and financial news headlines. Daily trading data, which are common predictors of stock price [94, 189], and technical indicator features were used in this model. There are opening price, closing price, high price, low price and volume and three technical indicators.

Table 3.3 shows some examples of filtered financial news headlines. To illustrate, 6423 headlines regarding Apple Inc. were extracted and then reduced to 2799 headlines after filtering via Reverb [190].

ReVerb is a program that automatically identifies and extracts binary relationships from English sentences [190]. ReVerb is designed for Web-scale information extraction, where the target relations cannot be specified in advance and speed is important. ReVerb takes raw text as input, and outputs (argument1, relation phrase, argument2) triples. For example, given the sentence "Bananas are an excellent source of potassium," ReVerb will extract the triple (bananas, be source of, potassium).

In this research, let us take the article "What is next for Apple's board?", published 
Chapter 3. Materials and frameworks design

Table 3.3: Samples extracted from the dataset.

\begin{tabular}{ccc}
\hline Date & News headline & News headline event tuple \\
\hline $3 / 18 / 2012$ & Chinese writers' group sues & Chinese writers' group, sues, \\
& Apple: state media & Apple \\
$4 / 13 / 2012$ & German court upholds ban on & German court, upholds ban \\
& Apple push email & on, Apple push email \\
$4 / 4 / 2012$ & Apple, two publishers hold out & Apple- two publishers, hold \\
& against settlement-report & out against, settlement-report \\
$3 / 26 / 2012$ & Apple CEO Cook meets & Apple CEO Cook, meets, \\
& Chinese officials & Chinese officials \\
$4 / 25 / 2012$ & US STOCKS-Apple results put & Apple, results, Nasdaq on \\
& Nasdaq on track for best gain & track for best gain \\
& of year & \\
\hline
\end{tabular}

Table 3.4: Training and text in the dataset.

\begin{tabular}{ccccc}
\hline $\begin{array}{c}\text { Data } \\
\text { source }\end{array}$ & Company & Training & Test & Total \\
\hline \multirow{4}{*}{ Reuters } & Apple & 780 & 161 & 941 \\
& Microsoft & 832 & 208 & 1040 \\
& Samsung & 855 & 214 & 1069 \\
\hline \multirow{3}{*}{ CNN } & Boeing & 528 & 132 & 660 \\
& Google & 1010 & 252 & 1262 \\
& Walmart & 798 & 200 & 998 \\
\hline
\end{tabular}

on 6 October 2011, as a specific example. The title of the article cannot be transformed into an event tuple using Reverb. Because the sentence is in the interrogative form, there is no event tuple that constitutes a rule. After the matching of time-wise data and stock data, 941 headlines were left. Daily news and stock data were aligned to create input-output pairs, except the days when no news was released. In 9 January 2012, for instance, three news articles were reported, but this study chose only one headline for alignment with stock market data. News events possibly happen several times within one day, but they do not happen every day, unlike stock trading, which happens daily, except on non-trading days falling on weekends or holidays. 
Table 3.5: Number of event characteristics labels for each company.

\begin{tabular}{ccccccc}
\hline Label & Apple & Microsoft & Samsung & Boeing & Google & Walmart \\
\hline 0 & 502 & 967 & 1433 & 133 & 661 & 206 \\
1 & 537 & 432 & 379 & 200 & 245 & 287 \\
2 & 661 & 557 & 378 & 152 & 389 & 233 \\
3 & 692 & 707 & 724 & 233 & 683 & 438 \\
4 & 407 & 228 & 193 & 147 & 196 & 180 \\
\hline Total & 2799 & 2891 & 3107 & 864 & 2174 & 1344 \\
\hline
\end{tabular}

\section{Data Preprocessing}

Table 3.4 shows that the matches found are pairs between event tuples and stock data. From this complete dataset, it used $80 \%$ of the samples in training data and the remaining $20 \%$ for testing data.

The following three steps are for preprocessing, which prepares for feature extraction and model creation.

(1) Tagging the label for each news headline. Five possible labels are characterized by categorical values as follows: 0 for an extremely negative label, 1 for a negative label, 2 to signify a neutral label, 3 for a positive label, and 4 to denote an extremely positive label. According to the time characteristics of each news headline, an event is manually tagged with the label for each news headline. Table 3.5 shows the labeling applied for each company: Label 0 means that a company's competitor has happened in this event; label 1 means that the company lost something; label 2 means that it did not cause any impact on the company; label 3 means that this event enabled the company to obtain something; and label 4 means that the company increased its profits or created more value.

(2) Word vector transformation. It used the word2vec [161 algorithm to train word embedding, and set the number of dimensions to 300. The word embedding was also trained using the Google News dataset, which contains 100 billion words that are characterized by a continuous bag-of-words structure.

(3) Technical indicators calculation. Three additional technical indicators that are calculated on the basis of daily trading data were used as follows:

- Stochastic oscillator $(\% \mathrm{~K})$. This indicator is a momentum analysis method created by 
George C. Lane. When the price trend rises, the closing price tends to approach the highest price of the day. When the price trend declines, the closing price tends to approach the lowest price of the day [191].

- Larry Williamss \%R indicator. This indicator is an oscillation indicator that measures the ratio of the highest price to the daily closing price. It indicates the proportion of stock price fluctuations in a certain period, thereby providing a signal out of the reversal of a stock market trend [192].

- Relative strength index (RSI). Buying and selling intentions in the market are analyzed by comparing the closing prices in a given period. Stocks that have had more or stronger positive changes have a higher RSI than do those that have had more or stronger negative changes. Strength indicators fall between 0 and 100; investors sell if this value is $\geq 80$ and buy if it is $\leq 20$ 94. 191 .

\subsubsection{Application scenario 2}

\section{UGC from social media}

UGC can be divided into structured and unstructured data in UGC. In this case, this study adopted two data types for modeling. UGC in Figure 3.2 contains structured data such as a movie's name, studio, revenue of movie, budget, theater, likes and shares, etc. Table 3.6 explains all the variables of structured data in the sample. The obtained information is from Facebook and Box Office Mojo from January 2016 to December 2017. This study selected 68 movies with average opening-weekend box-office revenues of $\$ 42.9$ million. Meanwhile, this study designed a web crawler by Python with Scrapy, which captures the audience's review from IMDb. Figure 3.3 shows an example of unstructured data, which mainly contains the audience's reviews.

The data sample was created as follows: (a) This study kept the movie for one month before its release and one month after its release (see Figure 3.4); (b) for each movie, this 


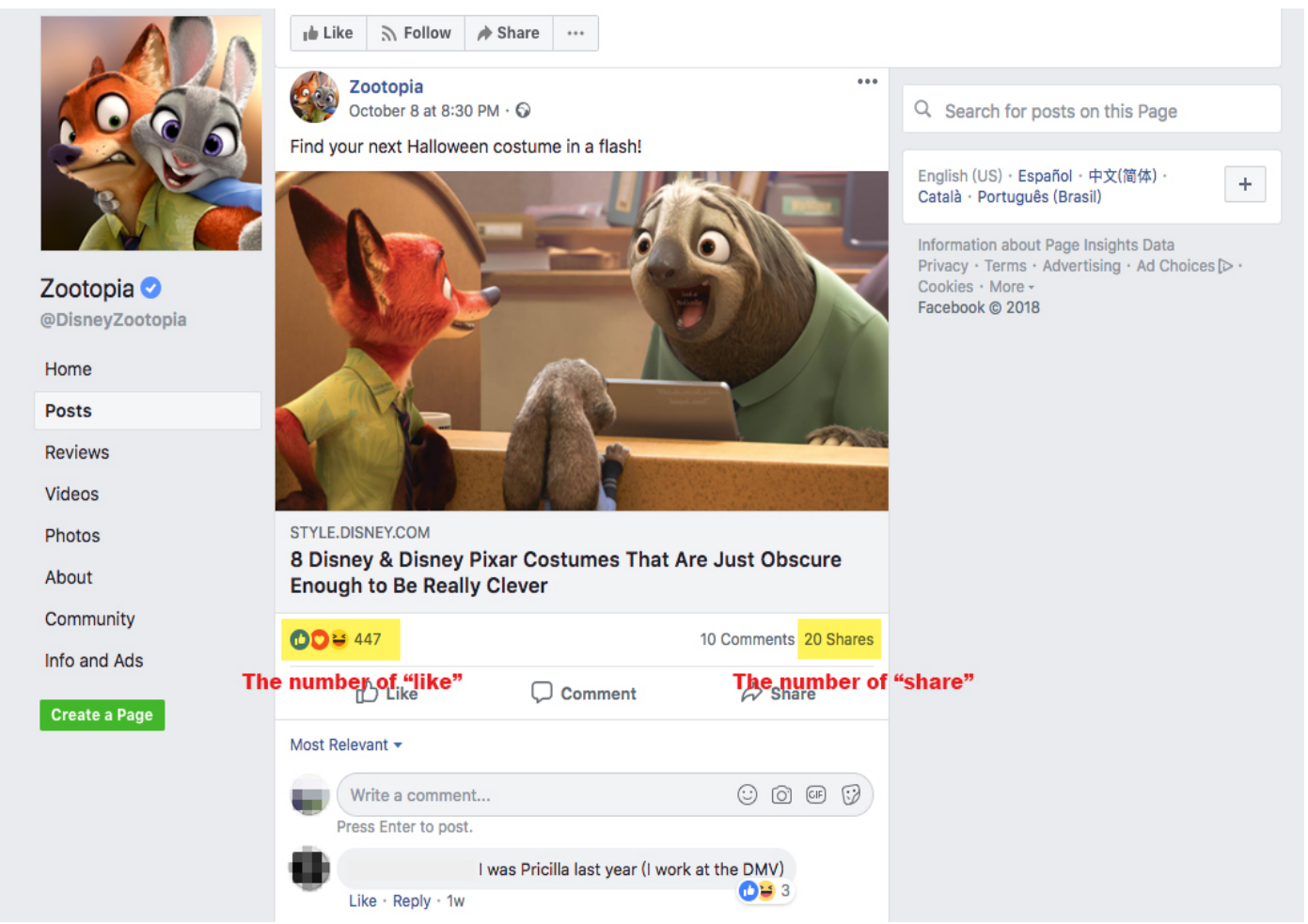

Figure 3.2: (Color online) an example of UGC on Facebook 5
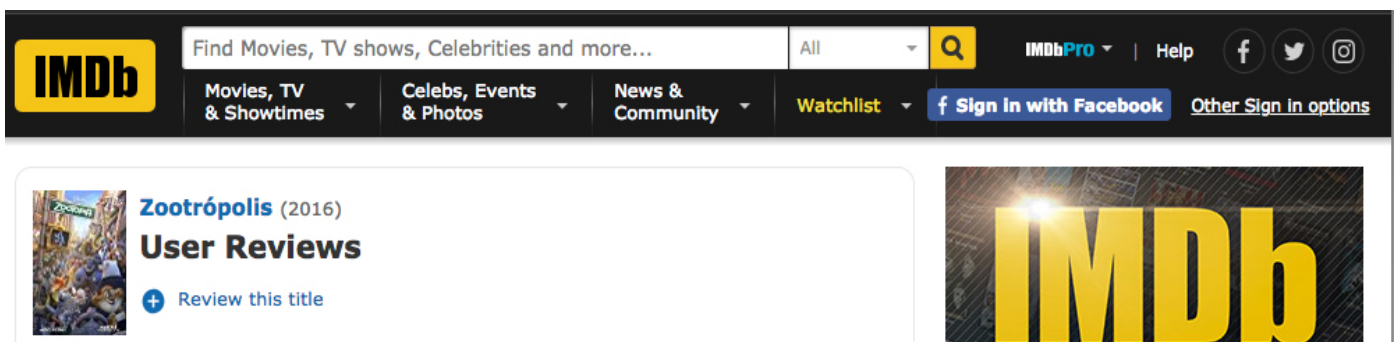

579 Reviews The number of review

Hide Spoilers Filter by Rating: Show All $\uparrow$ Sort by: Helpfulness $\downarrow \downarrow$
$10 / 10$ The rating

Best Disney movie made ever!

sennotaphoorn-41473 9 January 2018 The time The review

How can someone not like this masterpiece!

the characters they created are awesome! the world they created is mindblowing! the story is good!

Everything about this movie is just awesome!

26 out of 29 found this helpful. Was this review helpful? Sign in to vote.

Permalink

The Best Film of The Year, So Far.

CinemaClown 2 June 2016

From the studio that brought you Tangled, Wreck-It Ralph, Frozen \& Big Hero 6, Zootopia rurfarno ar a hinhlo

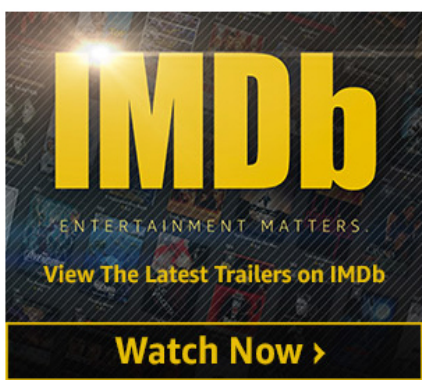

ad feedback

Zootrópolis

Opinion

Awards

FAQ

User Reviews

User Ratings

External Reviews

Metacritic Reviews

$\approx$ Explore More

Share this page: $f 0^{\circ}$

Figure 3.3: An example of Zootopia's IMDb page [6]. 
Table 3.6: Definition of variables.

\begin{tabular}{|c|c|c|}
\hline Variable & Description & Data source \\
\hline Rev1 & Total box office on the opening day & Box Office Mojo \\
\hline Rev7 & Total box office in the opening week & Box Office Mojo \\
\hline Rev30 & Total box office in the opening month & Box Office Mojo \\
\hline Budget & The budget for the movie investment & Box Office Mojo \\
\hline Thea1 & Number of theaters on the opening day & Box Office Mojo \\
\hline Thea7 & Number of theaters on the opening week & Box Office Mojo \\
\hline Thea30 & Number of heaters on the opening month & Box Office Mojo \\
\hline MAPP & Movie's MPAA rating & Box Office Mojo \\
\hline Genre & Movie's genre or type & Box Office Mojo \\
\hline Friday & The movie release on Friday & Box Office Mojo \\
\hline Pre_like1 & $\begin{array}{c}\text { The accumulated number of likes the day } \\
\text { before the release }\end{array}$ & Facebook \\
\hline Pre_like7 & $\begin{array}{l}\text { The accumulated number of likes in the week } \\
\text { before the release }\end{array}$ & Facebook \\
\hline Pre_like30 & $\begin{array}{l}\text { The accumulated number of likes in the } \\
\text { month before the release }\end{array}$ & Facebook \\
\hline Like1 & $\begin{array}{c}\text { The accumulated number of likes the day } \\
\text { after the release }\end{array}$ & Facebook \\
\hline Like7 & $\begin{array}{c}\text { The accumulated number of likes in the week } \\
\text { after the release }\end{array}$ & Facebook \\
\hline Like30 & $\begin{array}{l}\text { The accumulated number of likes in the } \\
\text { month after the release }\end{array}$ & Facebook \\
\hline Pre_sharet1 & $\begin{array}{l}\text { The accumulated number of shares the day } \\
\text { before the release }\end{array}$ & Facebook \\
\hline Pre_share7 & $\begin{array}{l}\text { The accumulated number of shares in the } \\
\text { week before the release }\end{array}$ & Facebook \\
\hline Pre_share30 & $\begin{array}{l}\text { The accumulated number of shares in the } \\
\text { month before the release }\end{array}$ & Facebook \\
\hline Share1 & $\begin{array}{l}\text { The accumulated number of shares the day } \\
\text { after the release }\end{array}$ & Facebook \\
\hline Share7 & $\begin{array}{c}\text { The accumulated number of shares in the } \\
\text { week after the release }\end{array}$ & Facebook \\
\hline Share30 & $\begin{array}{c}\text { The accumulated number of shares in the } \\
\text { month after the release }\end{array}$ & Facebook \\
\hline Return1 & $\begin{array}{c}\text { The stock return of the studio the day after } \\
\text { the release }\end{array}$ & Yahoo Finance \\
\hline Return7 & $\begin{array}{l}\text { The stock return of the studio in the week } \\
\text { after the release }\end{array}$ & Yahoo Finance \\
\hline Return30 & $\begin{array}{l}\text { The stock return of the studio in the mouth } \\
\text { after the release }\end{array}$ & Yahoo Finance \\
\hline Re_sp1 & $\begin{array}{c}\text { The stock return of the } \mathrm{S} \& \mathrm{P} 500 \text { the day } \\
\text { after the release }\end{array}$ & Yahoo Finance \\
\hline Re_sp7 & $\begin{array}{c}\text { The stock return of the } \mathrm{S} \& \mathrm{P} 500 \text { in the week } \\
\text { after the release }\end{array}$ & Yahoo Finance \\
\hline Re_sp30 & $\begin{array}{l}\text { The stock return of the } \mathrm{S} \& \mathrm{P} 500 \text { in the } \\
\text { month after the release }\end{array}$ & Yahoo Finance \\
\hline
\end{tabular}




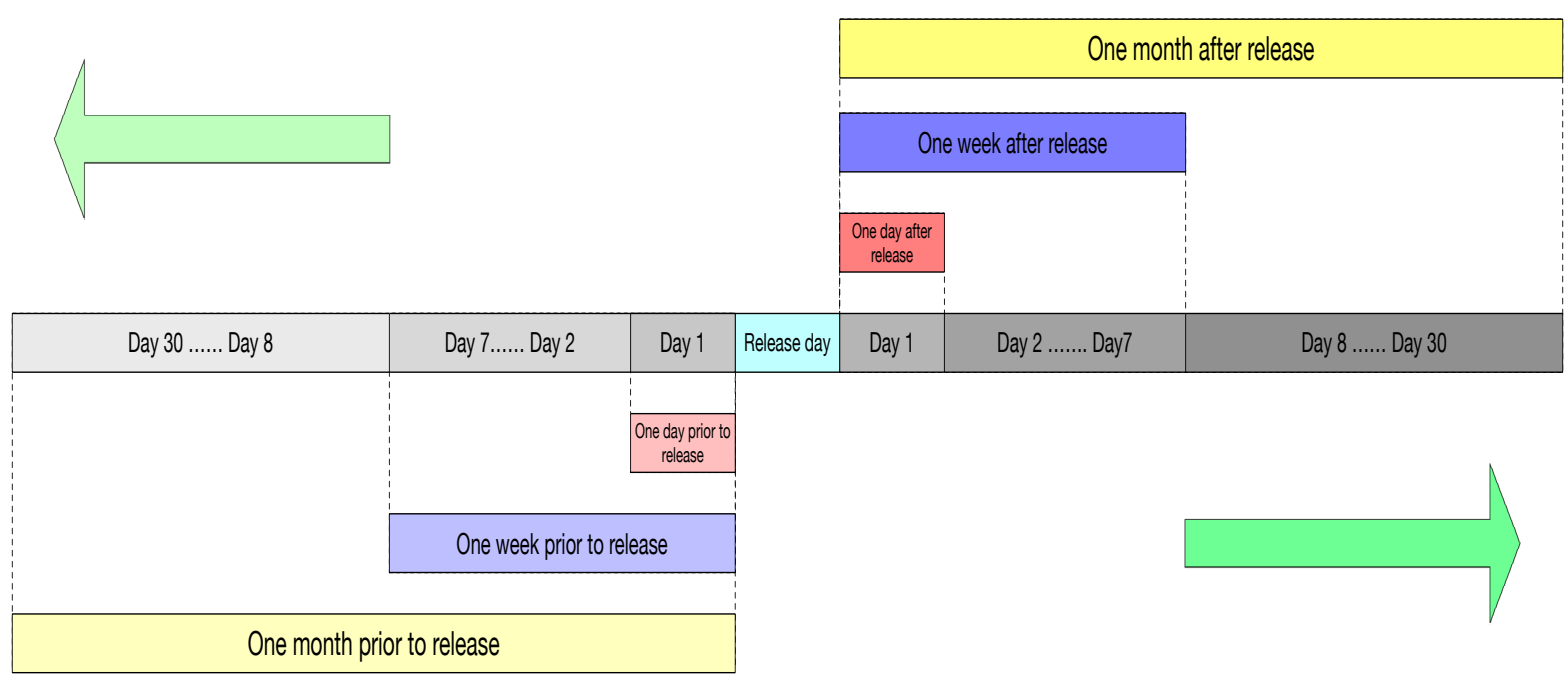

Figure 3.4: The timing of data collection periods.

Table 3.7: Quotes and studio names.

\begin{tabular}{ccc}
\hline Studio & Studio name & Stock \\
\hline Disney & The Walt Disney Studio & DIS \\
Fox & Twentieth Century Fox Film & FOX \\
& Corporation & \\
Lionsgate & Lions Gate Entertainment & LGF.A \\
& Corporation & \\
Paramount & Paramount Pictures Corporation & PGRE \\
Sony/Columbia & Sony/Columbia Pictures & SNE \\
Universal & Universal Studios & UVV \\
Warner & Warner Bros. Entertainment, Inc. & TWX \\
\hline
\end{tabular}

study collected the total box office in the opening day, opening week, opening month and the gross revenue of the USA from Box Office Mojo; (c) This study computed the total number of likes and shares on Facebook; (d) This study gathered the audience's review of the movie from one studio; for instance, Beauty and the Beast, Black Panther and Zootopia were produced by Disney; hence, this study combined their audiences' reviews in the same corpus.

\section{Stock data}

The historical price of stock data is from Yahoo Finance for seven studios. The lists of the studio names and stock quotes are shown in Table 3.7, which contains Hollywood's 
Chapter 3. Materials and frameworks design

Table 3.8: Training data and testing data in UGC.

\begin{tabular}{ccccccc}
\hline & \multicolumn{3}{c}{ box office } & \multicolumn{3}{c}{ Stock price movement } \\
\hline & Training & Testing & Total & Training & Testing & Total \\
\hline Disney & 7215 & 1840 & 9019 & 4629 & 1157 & 5786 \\
Fox & 5580 & 1395 & 6975 & 3534 & 883 & 4417 \\
Lionsgate & 1252 & 313 & 1565 & 826 & 207 & 1033 \\
Paramount & 2203 & 551 & 2754 & 1368 & 342 & 1710 \\
Sony/Columbia & 1326 & 332 & 1658 & 854 & 213 & 1067 \\
Universal & 3450 & 862 & 4312 & 2209 & 552 & 2761 \\
Warner & 5381 & 1345 & 6726 & 3378 & 844 & 4222 \\
\hline
\end{tabular}

major movie studios and are representative of the American movies. Each studio's stock data consists of open price, high price, low price, close price and adjusted close price in each transaction date. The stock return of the studios is calculated through the close price on a different day, defined thus:

$$
\text { Stock return }{ }_{t}=\log \frac{\text { close price }_{t+1}}{\text { close price }{ }_{t}}
$$

where close $_{\text {price }}$ is at day t; the rest stock data can be done in the same manner. The stock market is only open on trading days (excluding weekends and holidays). If the release date or calculation date falls on a non-trading day, this study needs to push back the matching date one or two days.

\section{Data pre-processing}

Table 3.8 shows the review corpus information in two aspects. The data set contains audiences' reviews published by IMDb; it also consists of the audiences' reviews published at specific time intervals, with each review report accompanied by a title, a release date, the audience's rating and the review content. This study tagged the audiences' reviews for the classification of the audiences' sentiment through the rating-based method. The audience's rating means that the sentiment of a specific review is negative; thus, the label is given a zero (0). In contrast, the audience's rating greater than or equal to 5 means that the sentiment 
of a particular review is positive; thus, the label is given a one (1).

This study tagged each review through the trading date of the studio's stock. The release date of the review was used to align each corresponding trading day from a temporal series; This study removed non-trading days such as weekends and holidays. Thus, the number of reviews in the aspect of stock price movement was less than the number of reviews in the aspect of box office revenue. The target output consisted of a binary variable, where a value of 1 indicated that the closing price at day $t+1$ would be higher than that at day $\mathrm{t}$, and a value of 0 indicated that the closing price at day $t+1$ would be lower than that at day t. In the balanced distribution of two classes for review corpus, this study split the data into training and testing data set. This study sequentially partitioned $80 \%$ of the samples for training reviews and the remaining $20 \%$ for testing reviews, which measured the classification performance. Hence, Disney, for instance, consisted of 7215 training reviews and 1840 testing reviews in the aspect of the box office revenue. Meanwhile, consisted of 4629 training reviews and 1157 testing reviews in the aspect of the stock price movement.

\section{Summary statistics}

Table 3.9 shows the summary statistics of the variables. On the opening day, the average box office occupies $12.38 \%$ of total box office in the USA, and on the opening week, the average box office occupies $42.47 \%$ of total box office in the USA; similarly, in the opening month, the average box office occupies $87.43 \%$ of total box office in the USA. However, during the opening month, the difference is $31.65 \%$ above two periods. Meanwhile, in the opening month, $\$ 88.3$ million is twice as much as $\$ 42.9$ million in the opening week. The number of likes on Facebook is $32,476.51,102,626.9$ and 430,293.4 for one day before the release, one week before the release and one month before the release, respectively. These figures show that, when the movie is at the 'before release' stage, the audience's mood is very positive about the movie, with the number of likes being $38,939.82,103,861.3$ and $175,368.3$ on the opening day, opening week and opening month, respectively. The comparison between 'before release' and 'after release' shows that the number of likes on the opening day is 6463.31 more than the day before the release. However, the number of likes in one month before the release 
Table 3.9: Summary statistics of structured data in UGC.

\begin{tabular}{cccccc}
\hline Variable & Mean & Min & Medium & Max & SD \\
\hline Rev1 & 12500000 & 30388 & 4752201 & 105000000 & 18700000 \\
Rev7 & 42900000 & 94970 & 18900000 & 297000000 & 58700000 \\
Rev30 & 88300000 & 2621711 & 39900000 & 588000000 & 116000000 \\
Rev_USA & 101000000 & 2716368 & 45000000 & 620000000 & 134000000 \\
Budget & 73.99559 & 1 & 50 & 250 & 63.22177 \\
(million) & & & & & \\
Thea1 & 2944.662 & 4 & 3147.5 & 4529 & 1188.98 \\
Thea7 & 2976.044 & 4 & 3151.5 & 4348 & 1166.024 \\
Thea30 & 1388.426 & 1 & 943.5 & 3801 & 1228.113 \\
Pre_like1 & 32476.51 & 211 & 6612.5 & 480000 & 72325.47 \\
Pre_like7 & 102626.9 & 1144 & 30237 & 970578 & 195295.6 \\
Pre_like30 & 430293.4 & 1897 & 135050 & 7214487 & 981735.2 \\
Like1 & 38939.82 & 0 & 8179 & 459391 & 76245.68 \\
Like7 & 103861.3 & 900 & 22125.5 & 1191429 & 212388.5 \\
Like30 & 175368.3 & 1324 & 29320 & 2018829 & 350184.3 \\
Pre_share1 & 4735.206 & 25 & 1127 & 94658 & 12296.78 \\
Pre_share7 & 18762.28 & 88 & 5388 & 225044 & 36671.27 \\
Pre_share30 & 94202.32 & 614 & 28288.5 & 1883679 & 249366.2 \\
Share1 & 7557.412 & 0 & 1324.5 & 230848 & 28651.31 \\
Share7 & 15883.46 & 131 & 3189.5 & 292262 & 47439.44 \\
Share30 & 22582.07 & 179 & 5240.5 & 342462 & 54874.87 \\
Return1 & 0.0010373 & -0.0156187 & 0 & 0.0170969 & 0.0053676 \\
Return7 & 0.0033474 & -0.0433776 & 0.0032337 & 0.0424593 & 0.014053 \\
Return30 & 0.0059519 & -0.0664018 & 0.0074605 & 0.0689492 & 0.0259819 \\
Re_sp1 & 0.0006578 & -0.0081014 & 0.000129 & 0.0083949 & 0.0023431 \\
Re_sp7 & 0.0018791 & -0.0200381 & 0.0007342 & 0.0205774 & 0.0057159 \\
Re_sp30 & 0.0088256 & -0.0129019 & 0.006982 & 0.0430413 & 0.0101055 \\
\hline
\end{tabular}


Table 3.10: The differences of models are used traditional machine learning and deep learning.

\begin{tabular}{cccccc}
\hline Studios & Min & Mean & Max & SD & $\begin{array}{c}\text { Trading } \\
\text { days }\end{array}$ \\
\hline Disney & 1 & 8.18 & 415 & 20.86 & 1134 \\
Fox & 1 & 10.04 & 334 & 22.34 & 786 \\
Lionsgate & 1 & 2.92 & 47 & 3.94 & 598 \\
Paramount & 1 & 4.07 & 54 & 4.43 & 780 \\
Sony/Columbia & 1 & 3.93 & 51 & 5.56 & 610 \\
Universal & 1 & 5.64 & 100 & 7.88 & 760 \\
Warner & 1 & 8.23 & 178 & 13.66 & 961 \\
\hline
\end{tabular}

is $254,925.1$ more than the opening month after the release. The emotional performance of an audience that has already watched a movie exceeds expectations compared to the time before the movie's release. As time passes, the audience's interest begins to decline.

\subsubsection{Application scenario 3}

\section{Audience review in UGC}

Audience reviews for this work were collected from IMDb (https://www.imdb.com/), which provides a large amount of data related to movie information. IMDb is the most wellknown online review site. It designed a web crawler by Python with Scrapy, which captures these reviews, a total 60,012 reviews from 95 movies between 4 November 2013 and 26 July 2018. It also consists of the review published at specific time intervals, with each review report accompanied by a title, a release date, and audience rating.

\section{Stock data}

The list of studio names is same application scenario 2, which shown in Table 3.7.

\section{Data pre-processing}

This study gathered audience reviews of movies from the same studio from IMDb. For instance, A Cure for Wellness, Goodbye Christopher Robin, and The Mountain Between Us 
Chapter 3. Materials and frameworks design

Table 3.11: Information of review corpus in two cases of studios' performance.

\begin{tabular}{ccccccc}
\hline & \multicolumn{3}{c}{ box office } & \multicolumn{3}{c}{ Stock price movement } \\
\hline & Training & Testing & Total & Training & Testing & Total \\
\hline Disney & 12124 & 3031 & 15155 & 7420 & 1855 & 9275 \\
Fox & 9958 & 2490 & 12448 & 6316 & 1579 & 7895 \\
Lionsgate & 2194 & 548 & 2741 & 1399 & 350 & 1749 \\
Paramount & 4879 & 1220 & 6099 & 2540 & 635 & 3175 \\
Sony/Columbia & 2959 & 740 & 3699 & 1918 & 480 & 2398 \\
Universal & 5810 & 1453 & 7263 & 3430 & 858 & 4288 \\
Warner & 10085 & 2521 & 12606 & 6326 & 1581 & 7907 \\
\hline
\end{tabular}

Table 3.12: Time interval in training data and testing data.

\begin{tabular}{cccccc}
\hline & & \multicolumn{2}{c}{ box office } & \multicolumn{2}{c}{ Stock price movement } \\
\hline & Time interval & Training & Testing & Training & Testing \\
\hline Disney & $18.4 .2013-$ & $18.4 .2013-$ & $8.1 .2018-$ & $18.4 .2013-$ & $22.12 .2017-$ \\
& 17.4 .2018 & 8.1 .2018 & 17.4 .2018 & 22.12 .2017 & 17.4 .2018 \\
Fox & $9.5 .2014-$ & $9.5 .2014-$ & $25.12 .2017-$ & $9.5 .2014-$ & $20.12 .2017-$ \\
& 24.7 .2018 & 25.12 .2017 & 24.7 .2018 & 20.12 .2017 & 24.7 .2018 \\
Lionsgate & $11.11 .2013-$ & $11.11 .2013-$ & $15.9 .2017-$ & $11.11 .2013-$ & $15.9 .2017-$ \\
& 25.7 .2018 & 25.7 .2018 & 25.7 .2018 & 15.9 .2017 & 25.7 .2018 \\
Paramount & $29.10 .2014-$ & $29.10 .2014-$ & $23.8 .2017-$ & $29.10 .2014-$ & $3.10 .2017-$ \\
& 26.7 .2018 & 23.8 .2017 & 26.7 .2018 & 3.10 .2017 & 26.7 .2018 \\
Sony/Columbia & $11.4 .2014-$ & $11.4 .2014-$ & $21.12 .2017-$ & $11.4 .2014-$ & $19.12 .2017-$ \\
& 25.7 .2018 & 21.12 .2017 & 25.7 .2018 & 18.12 .2017 & 25.7 .2018 \\
Universal & $13.5 .2013-$ & $13.5 .2013-$ & $22.8 .2017-$ & $13.5 .2013-$ & $8.9 .2017-$ \\
& 25.7 .2018 & 21.8 .2017 & 25.7 .2018 & 8.9 .2017 & 25.7 .2018 \\
Warner & $11.6 .2013-$ & $11.6 .2013-$ & $29.10 .2017-$ & $11.6 .2013-$ & $26.10 .2017-$ \\
& 14.7 .2018 & 29.10 .2017 & 26.7 .2018 & 26.10 .2017 & 14.6 .2018 \\
\hline \multirow{2}{*}{ W. } & & & & & \\
\hline
\end{tabular}


were produced by Fox; audience reviews were combined in the same corpus, which makes it easy to form a corpus of the same studio. The dataset contains audience reviews, audience ratings, and review content. This work tagged audience reviews through the rating-based method. An audience rating less than 5 means that the sentiment of this review is negative; then the label given is 0 . In contrast, if the audience rating is equal to or greater than 5 , the sentiment is positive, and the label given is 1 .

Table 3.10 shows matching statistics between audience reviews and stock prices for each studio. The release date of the review is used to align the corresponding trading day from a temporal series. Non-trading days were removed non-trading days, such as weekends and holidays; unpaired stock data and audience reviews were also removed, since the stock market only occurs on trading days (except weekends and holidays). If the release date or calculation date happened on a non-trading day, this study pushed the release date back one day or two days.

There were fewer reviews associated with the stock market than associated with audience sentiment. The target output consists of a binary variable, where a value of 1 indicates that the closing price that day $\mathrm{t}+1$ will be higher than that at day $\mathrm{t}$, and a value of 0 indicates that the closing price at day $\mathrm{t}+1$ will be lower than that at day $\mathrm{t}$, which is defined in the following equation:

$$
\hat{y}=\left\{\begin{array}{l}
0, p>0 \\
1, \text { else }
\end{array}\right.
$$

where $\hat{y}$ represents the predicted label in each candidate pair, and $p$ denotes distribution probability. Afterward, according on the principle of time series, it split $80 \%$ of the samples for training review and the remaining $20 \%$ for testing review, which is shown in Table 3.11. Meanwhile, it also calculated the time interval of the review corpus in Table 3.12. 
Table 3.13: Features of the prediction model.

\begin{tabular}{cc}
\hline Feature & Feature expression \\
\hline Stock price yesterday + yesterday trend & price $_{t-1}$ and $t_{-}$indicator $_{t-1}$ \\
Same + Bag of words & price $_{t-1}$ and $t f_{-i d f_{t-1}}$ \\
Same + some features inferred through a & price $_{t-1}, t_{\text {_indicator }}$ $_{t-1}$ and \\
CNN & cnn $_{t-1}$ \\
Same + some features inferred through a & price $_{t-1}, t_{-}$indicator $_{t-1}$ and \\
Combined features & $f_{-}$combine $_{t-1}$ \\
\hline
\end{tabular}

\subsection{Framework design}

\subsubsection{Application scenario 1}

To assess the effectiveness of applying the prediction model on the basis of financial news, This study designed four sets of features for predicting stock price movement (Table 3.13). Features 3 and 4 are used for event characteristics. Each feature is explained in the succeeding subsections. The target output consists of a binary variable, for which a value of 1 indicates that the closing price at day $t+1$ will be higher than that at day $t$, and a value of 0 indicates that the closing price at day $t+1$ will be lower than that at day $t$.

\section{Stock data only}

This study considered historical price as the input for predicting stock price movement and used it as a baseline for comparison with other sets of features. The features used to train the machine learning model that uses only stock data are price $_{t-1}$ and $t \_$indicator $_{t-1}$. The output is the indicator of price movement (increase or decrease) at each transaction date examined.

\section{Bag of words}

The input feature set is based on a bag of words for the news and price trend for stock. This study first prepared each headline for data pre-processing and then transformed the pre-processed headline into a feature vector using the term frequency-inverse document frequency (TF-IDF) algorithm [193], which assigns high weight to eigenvectors. It estimates 


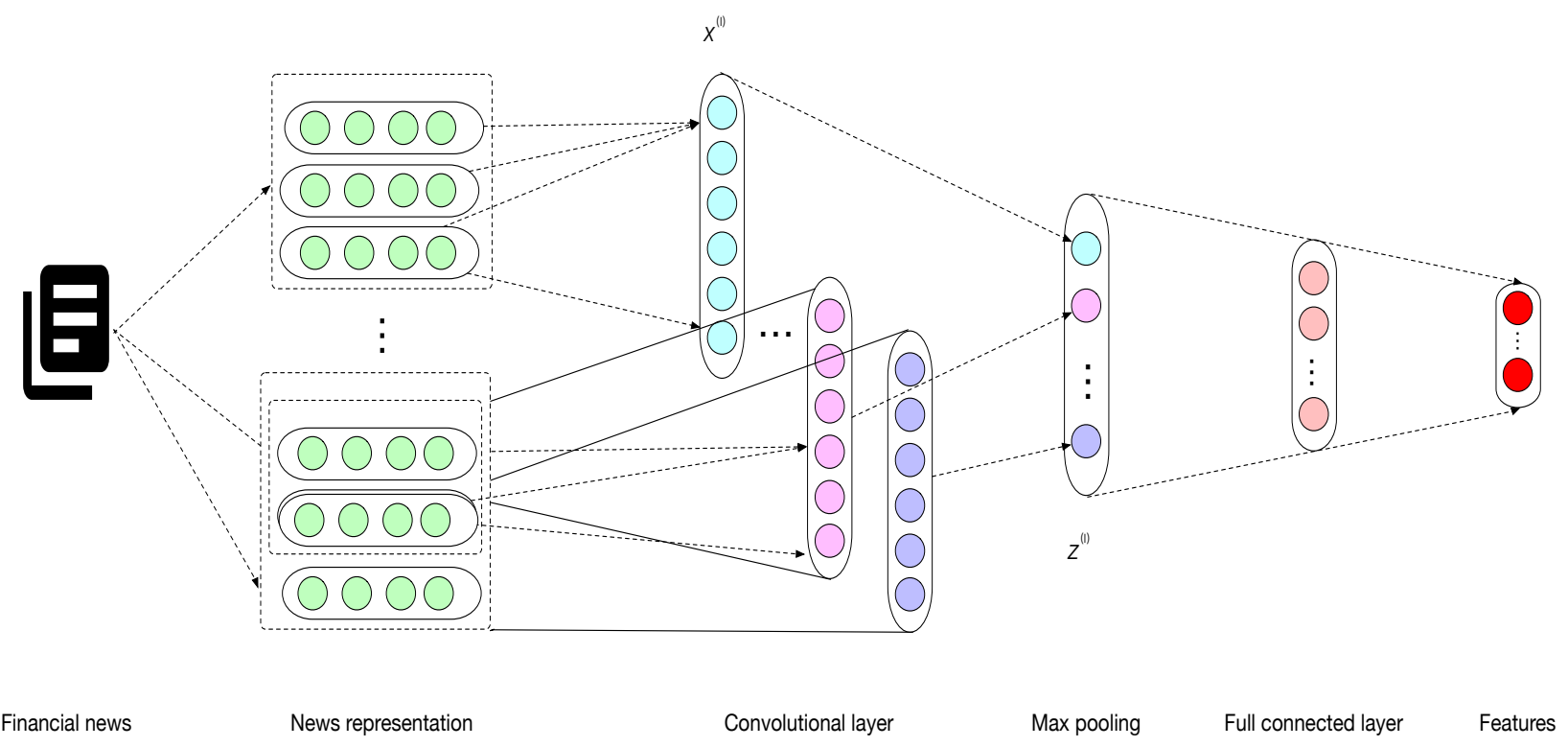

Figure 3.5: Convolutional neural network architecture proposed for feature selection.

the frequency of a term in one document over the maximum in a collection of documents and assesses the importance of a word in one set of documents. Such importance increases proportionally with the number of word appearances in a document. The features used to train the bag-of-words machine learning model are rrice $_{t-1}$ and $t f_{-} i d f_{t-1}$, which are the price movements (increase or decrease) at each transaction date examined.

\section{Convolutional neural network}

Given the sequence of words in a headline, the word2vec model 161 can be used to embed each of these words in a real valued-vector $\mathrm{x}$. In this work, This study set the dimension $\mathrm{n}$ of each word vector at 30 (i.e. $x \in \mathbb{R}^{30}$ ). This study concatenated the word vectors of all the words in a headline sequentially to form a matrix $X=\left[x^{(1)}, x^{(2)}, x^{(3)}, \ldots, x^{(4)}\right]$ as the input to a convolutional neural network model. For a headline with $\mathrm{N}$ words, the resultant input matrix has dimensions of $30 \times \mathrm{N}$, and the dimension of news representation also is 30 .

In Fig 3.5, this convolutional neural network model is made up of four consecutive layers: The first layer that is the input, the second layer that is convolutional, the third layer is a max-pooling and the last layer that is a fully connected layer. The convolutional and maxpooling layers were designed using the text-CNN [194], which effectively carries out sentiment 
classification. In the convolutional layer, input matrix $\mathrm{X}$ convolves with a kernel $\mathrm{w} \in \mathbb{R}^{n \times k}$, where $\mathrm{n}$ is the size of a word vector (30 in this work), and its dimension is $50 . \mathrm{k}$ denotes the size of a sliding window ( $\mathrm{k}=3$ in this study). The computation can be formulated as follows:

$$
Z_{i}=\sigma\left(w X_{i: i+k-1}+b\right)
$$

where $X_{i: i+k-1}=\left[x_{i}, x_{i+1}, \ldots, x_{i+k-1}\right]$ is the portion of input $\mathrm{X}$ falling within the sliding window, b denotes the optional offset and $\sigma($.$) is the sigmoid function.$

In the next step, this study used the pooling layer to reduce the convolutional neural networks parameter space and consequently minimize information loss in the pooling processing and capture the most important features. The eigenvector of the filter in the pooling layer is cut into 3 chunks and a maximum value is taken in each chunk, this study obtained 3 eigenvalues. Convolutional output vector $\mathrm{Z}$ was split into $\mathrm{p}$ windows, and only the maximum feature as 3 in each window was kept for passage onto the final fully connected layer. The fully connected layer is linear regression, and the output layer is the feature classification between 0 and 1 .

The convolutional neural network model is intended to extract a feature vector from the fully connected layer to describe the emotional characteristics of input headlines. Following the intuition that a good feature vector should lead to the accurate classification of the event characteristics of headlines, this study attached a softmax layer after the fully connected layer when the convolutional neural network model was trained. The entire model was trained to classify the five emotional labels that describe event characteristics, and the resultant model will be able to provide a meaningful emotional feature vector for each inputted headline. Meanwhile, this study defines the loss function of the convolutional neural network model as $E_{d}$. The features used to train this machine learning model are price $_{t-1}, t_{-}$indicator $_{t-1}$ and $c n n_{t-1}$, which are the price movements (increase or decrease) at corresponding transaction date. 


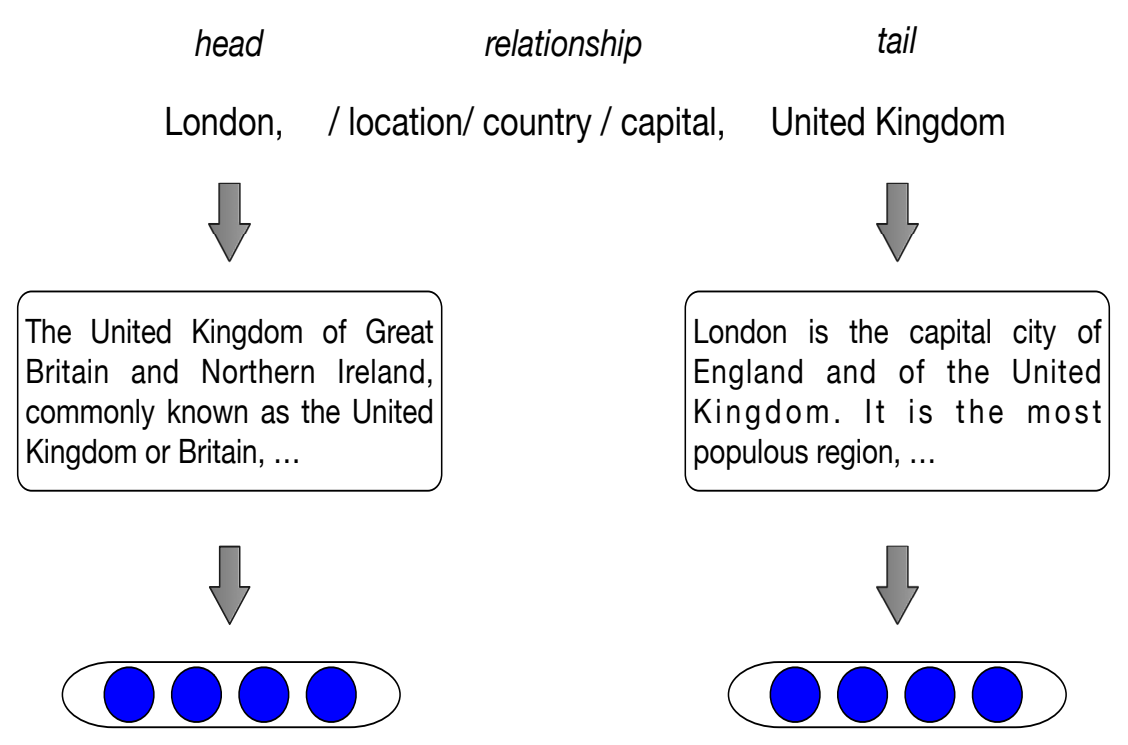

Figure 3.6: Text description of the entity.

\section{Combined features}

In the introduction section of this paper, this study described various translation models. Because the TransE model represents a one-to-one relationship between two entities [4], the relationship amongst many entities must be continuously incorporated into texts and knowledge graph [195]. This model combines a convolutional neural network with textual information extraction, which fully exploits the semantic information in a knowledge graph and text [196]. In Fig 3.6, knowledge graph contains rich semantics in entity description texts, but it is not fully utilized in feature extraction. Most of the existing text representation models simply train the text into word vectors through word2vec, which obtains the text representation by means of averaging etc. Hence, these ways often lose more semantic information. This study therefore proposed to extract feature vectors from news texts using a CNN model combined with the TransE model, which also fully integrates the two parts of the feature information.

In Fig 3.7, this architecture used in the feature combination model encompasses two parts, namely, a CNN and the TransE model. The feature combination sets are the average of each word vector in an entity and it was obtained using the word2vec model. The two entity vectors are mapped into the same relational space, and these entities using a trained 


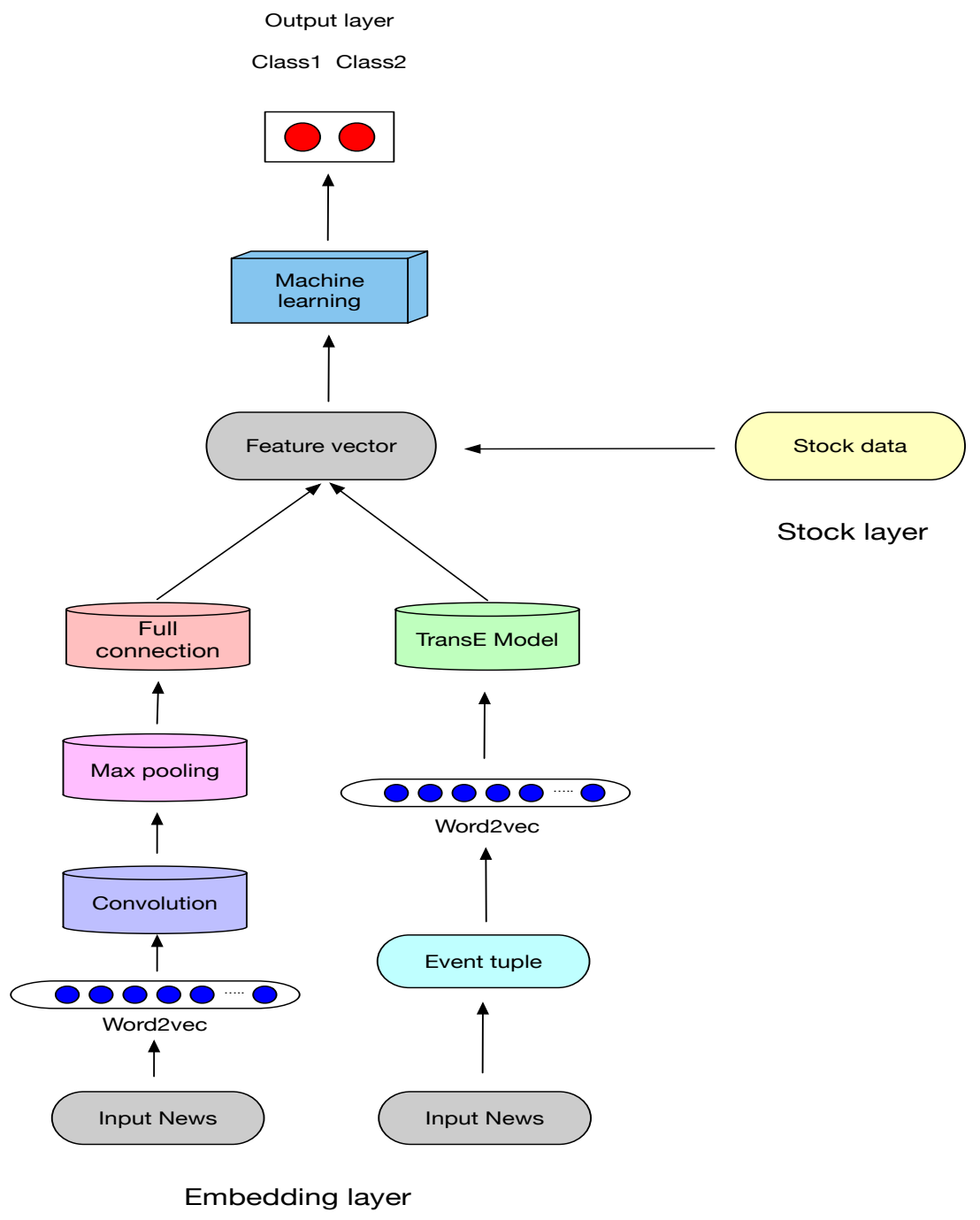

Figure 3.7: Proposed architecture for the feature combination model. 
low-rank weight matrix 197.

As assumed in the TransE model, relationship vector $\mathrm{R}$ should satisfy ${ }^{\mathfrak{u}} E+R \approx T^{\prime}$. The model can be represented as a parameter set $\emptyset=\left(L_{r}, R_{r}, \theta\right)$, where $\mathrm{X}$, E and $\mathrm{R}$ represent a word, an entity and a relation, respectively. And $\mathrm{r}$ are the mapping matrices for entities in the structural model and represents the weights of the convolutional neural network. For instance, the sentence as "Samsung sues Apple for infringement", so "Samsung + Apple $\approx$ sues". The loss function $E_{s}$ of this structural model is defined as follows:

$$
E_{s}=\|h+r-t\|_{2}^{2}
$$

where $\mathrm{h}, \mathrm{r}$ and $\mathrm{t}$ represent head entity, relationship and tail entity in event tuple, respectively.

And text representation $E_{d}$ is consistent with Section 3.2.2, which denotes:

$$
E_{d}=f_{r}\left(h_{s}, t_{d}\right)+f_{r}\left(h_{d}, t_{s}\right)+f_{r}\left(h_{d}, t_{d}\right)
$$

where $f_{r}\left(h_{d}, t_{d}\right)=\left\|h_{d}+r-t_{d}\right\|_{2}^{2}$ represent the head entity and the tail entity are in text representation. $f_{r}\left(h_{d}, t_{s}\right)=\left\|h_{d}+r-t_{s}\right\|_{2}^{2}$ and $f_{r}\left(h_{s}, t_{d}\right)=\left\|h_{s}+r-t_{d}\right\|_{2}^{2}$ represent one of the head entity $\mathrm{h}$ and the tail entity $\mathrm{t}$ in text representation, another one is in structure representation.

Moreover, This study combined two types of representation learning (convolutional neural network and feature combination) to map news titles; these vectors will be into feature vectors. The relationship vector $\mathrm{R}$ identical to the result of the feature extraction layer of the convolutional neural network in the structural model. Besides, This study combined this loss with the classification loss of the convolutional neural network using L2 regularization, 
which obtains the overall loss function $\mathrm{E}$ for feature selection, that is:

$$
E=E_{s}+\alpha E_{d}+\beta\|\emptyset\|^{2}
$$

where $\|\emptyset\|^{2}$ is a regular item. $\alpha$ and $\beta$ are hyperparameters, which measures the loss of text information and the weight of the regular items, respectively. $W$ denotes the convolutional kernels in the layer and $T^{\prime}$ is a negative sample set of $T$ [4].

$$
T^{\prime}=\left\{\left(h^{\prime}, r, t\right) \mid h^{\prime} \in E\right\} \cup\left\{\left(h, r, t^{\prime}\right) \mid t^{\prime} \in E\right\} \cup\left\{\left(h, r^{\prime} t\right) \mid t^{\prime} \in R\right\}
$$

where the head entity and tail entity are randomly replaced by an entity or relationship in another tuple. In particular, if the replaced tuple is already in T, it wouldn't be added to the negative sample. Since both $\mathrm{h}$ and $\mathrm{t}$ represent two types of entities, there are structurebased representations and text-based representations in interval-based loss functions. The stochastic gradient descent (SGD) is employed to minimize the above loss function.

In this structure, this study chooses the optimal parameters as followed: the learning rate of SGD $\lambda=0.001$, the dimension of the representation vector of entities and relationships $\mathrm{k}=100$, the word vector dimension of the entity description text $\mathrm{n}=100$, and batch size during training is 1440. Convolution layer window size is $W \in\{1,2,3\}$. This experiment is performed in 1000 iterations of training and the optimal parameters are based on the testing set.

Here, the features used for training machine learning model are price $_{t-1}, t_{-}$indicator $_{t-1}$ and $f_{-}$combine $_{t-1}$, which are the price movements (increase or decrease) at the corresponding transaction date.

\section{Python code of knowledge graph}

These codes explain the technical details of deep learning and knowledge graph extract feature form financial news. Software requirements is Tensorflow 1.4, Text-CNN is 
in https://github.com/dennybritz/cnn-text-classification-tf. The detailed code of the model is as follows:

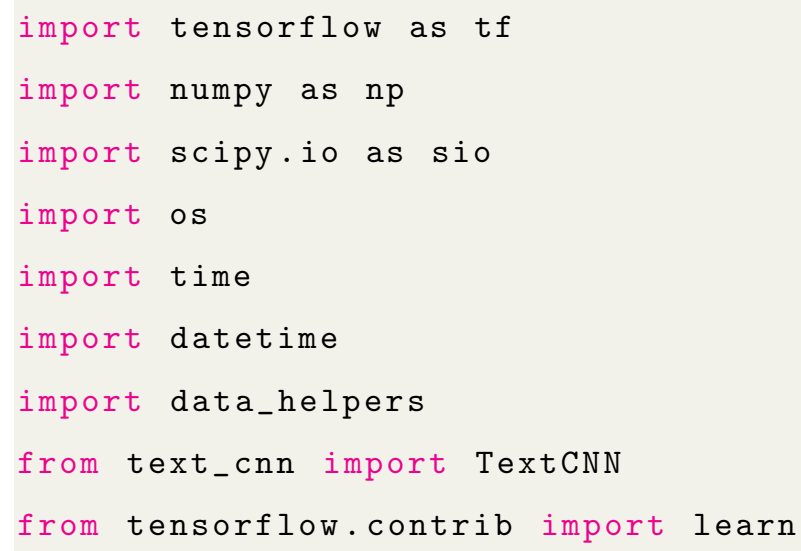

Listing 3.1: Input package.

Listing 3.1 shows each package input.

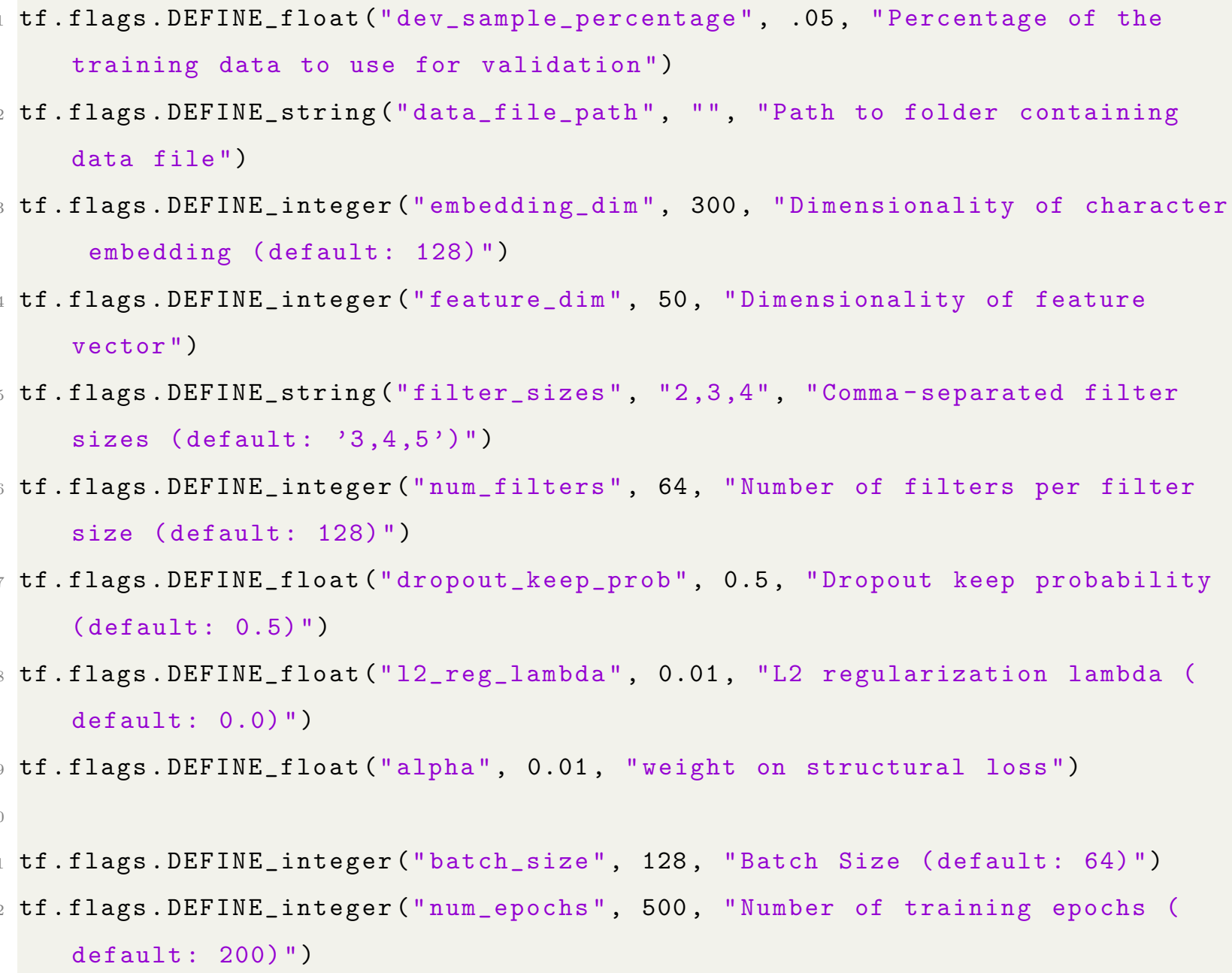


Chapter 3. Materials and frameworks design

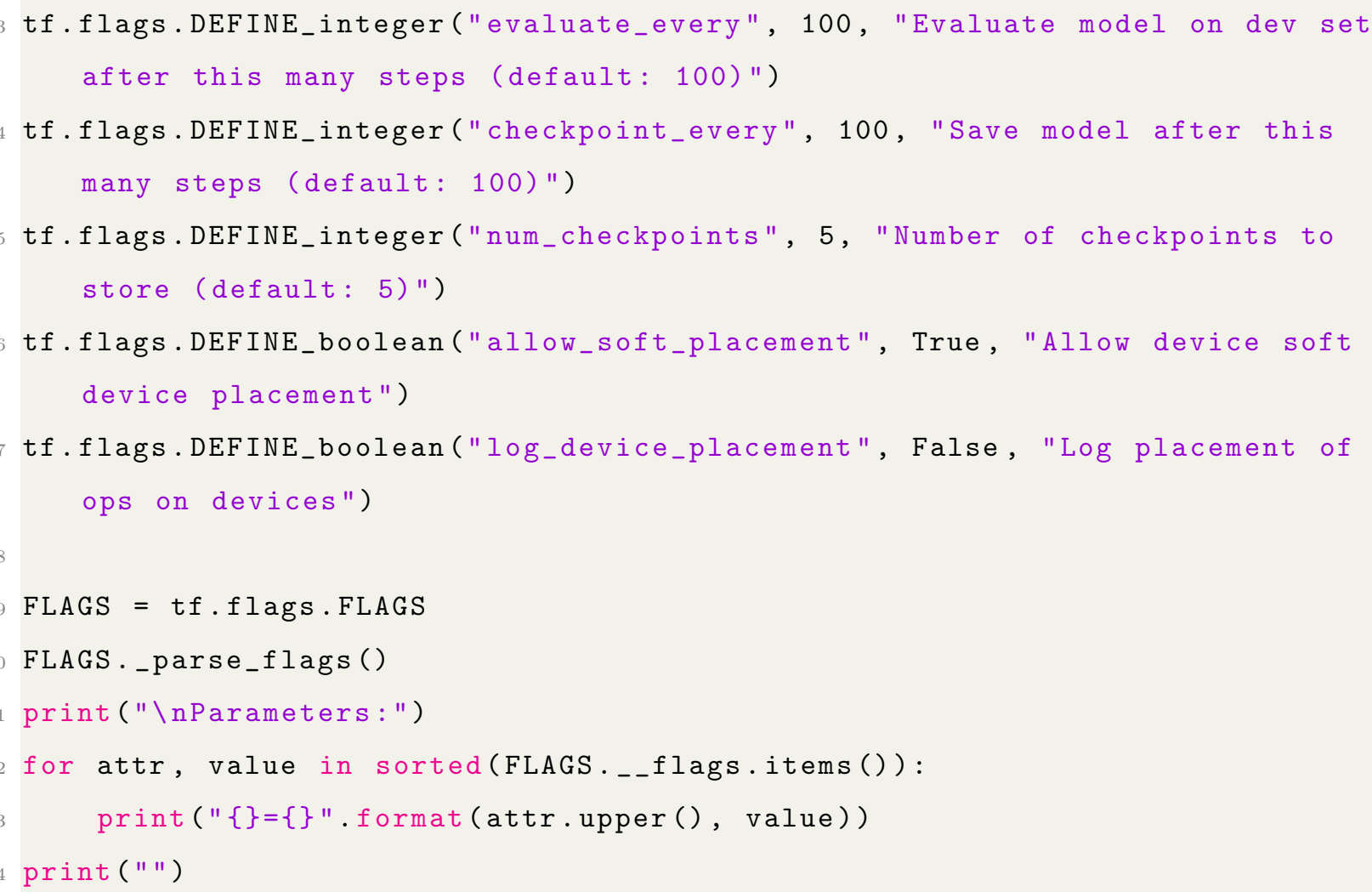

Listing 3.2: Definition parameters of hyperparameters and training.

Listing 3.2 defined model hyperparameters and training parameters.

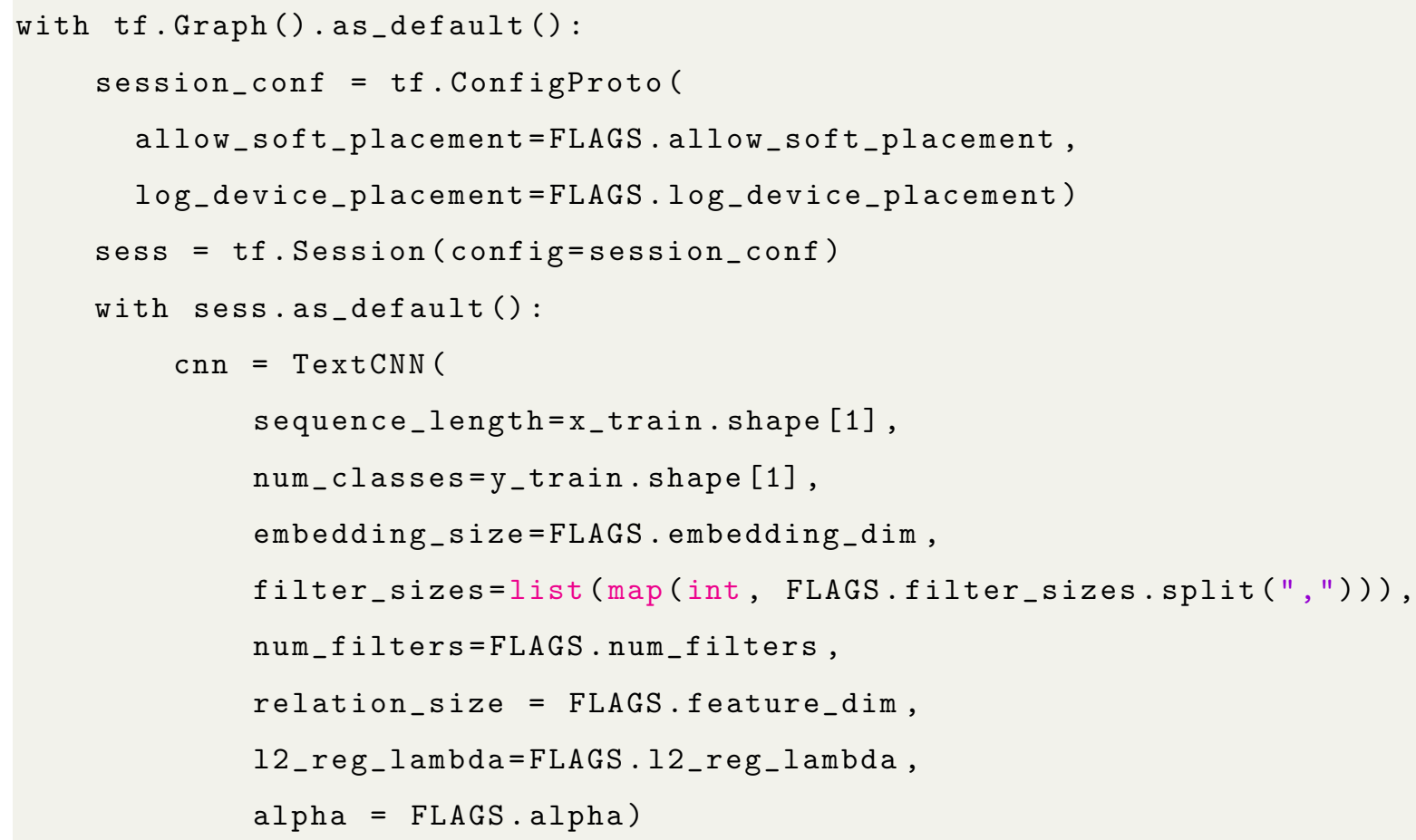


\# Define Training procedure

global_step = tf.Variable(0, name="global_step", trainable=False)

optimizer = tf.train.Adam0ptimizer $(1 \mathrm{e}-3)$

grads_and_vars = optimizer.compute_gradients(cnn.loss)

train_op = optimizer.apply_gradients(grads_and_vars, global_step= global_step)

\# Keep track of gradient values and sparsity (optional)

grad_summaries $=[]$

for $g, v$ in grads_and_vars:

if $\mathrm{g}$ is not None:

grad_hist_summary = tf.summary.histogram("\{\}/grad/hist".

format (v.name), g)

sparsity_summary = tf.summary.scalar (" \{\}$/ \operatorname{grad} /$ sparsity".

format (v.name), tf.nn.zero_fraction (g))

grad_summaries.append (grad_hist_summary)

grad_summaries.append (sparsity_summary)

grad_summaries_merged = tf.summary.merge(grad_summaries)

\# Output directory for models and summaries

timestamp $=\operatorname{str}($ int (time.time ()) )

out_dir = os.path.abspath(os.path.join(os.path.curdir, "runs", timestamp))

print("Writing to \{\}$\backslash n "$.format(out_dir))

\# Summaries for loss and accuracy

loss_summary = tf.summary.scalar("loss", cnn.loss)

acc_summary = tf.summary.scalar ("accuracy", cnn.accuracy)

\# Train Summaries

train_summary_op = tf.summary.merge([loss_summary, acc_summary, grad_summaries_merged])

train_summary_dir = os.path.join(out_dir, "summaries", "train")

train_summary_writer = tf.summary.FileWriter(train_summary_dir, sess.graph) 
Chapter 3. Materials and frameworks design

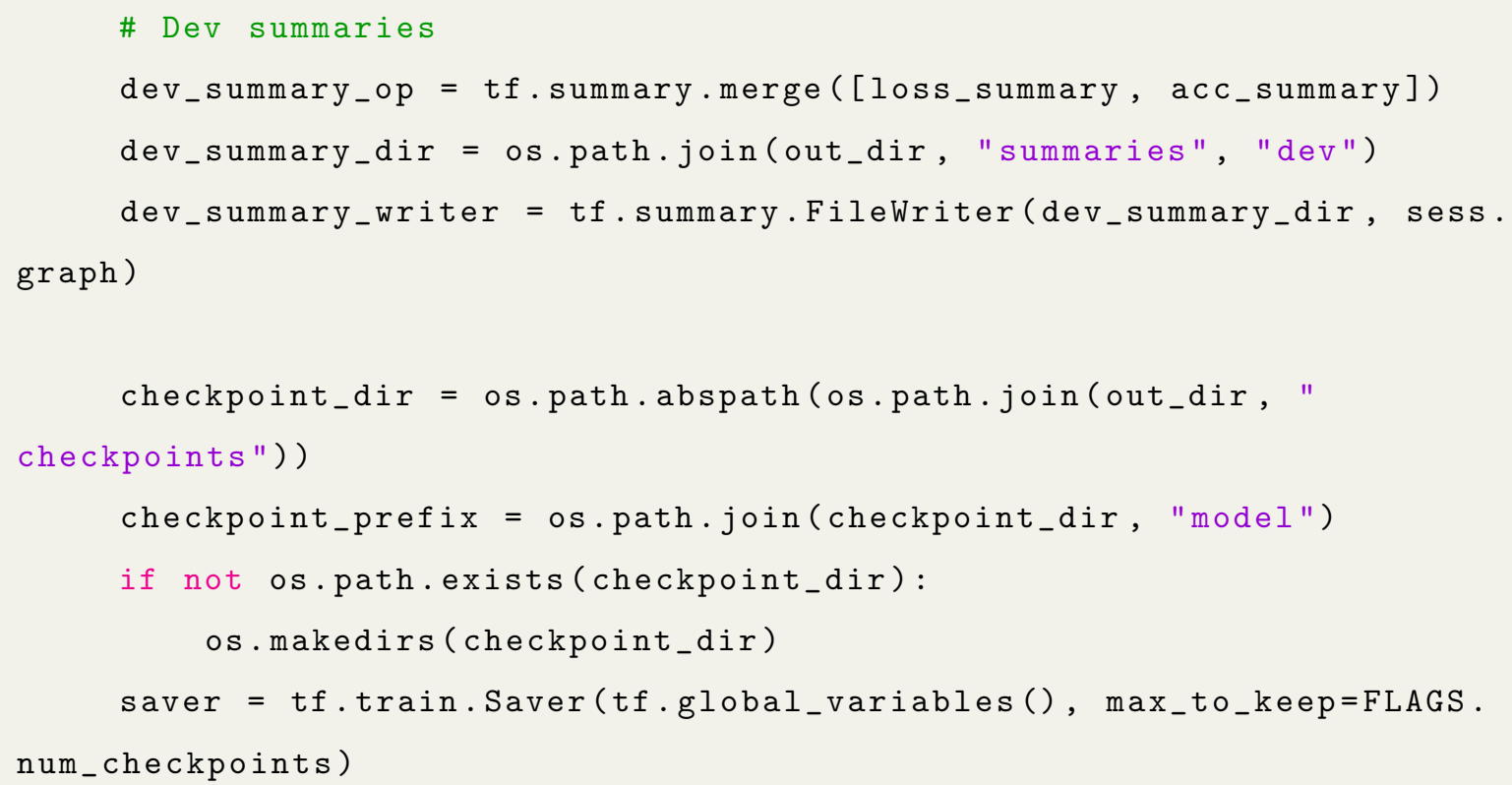

Listing 3.3: Definition parameters of hyperparameters and training.

Listing 3.3 describes the process of data training, which laid the foundation for the model.

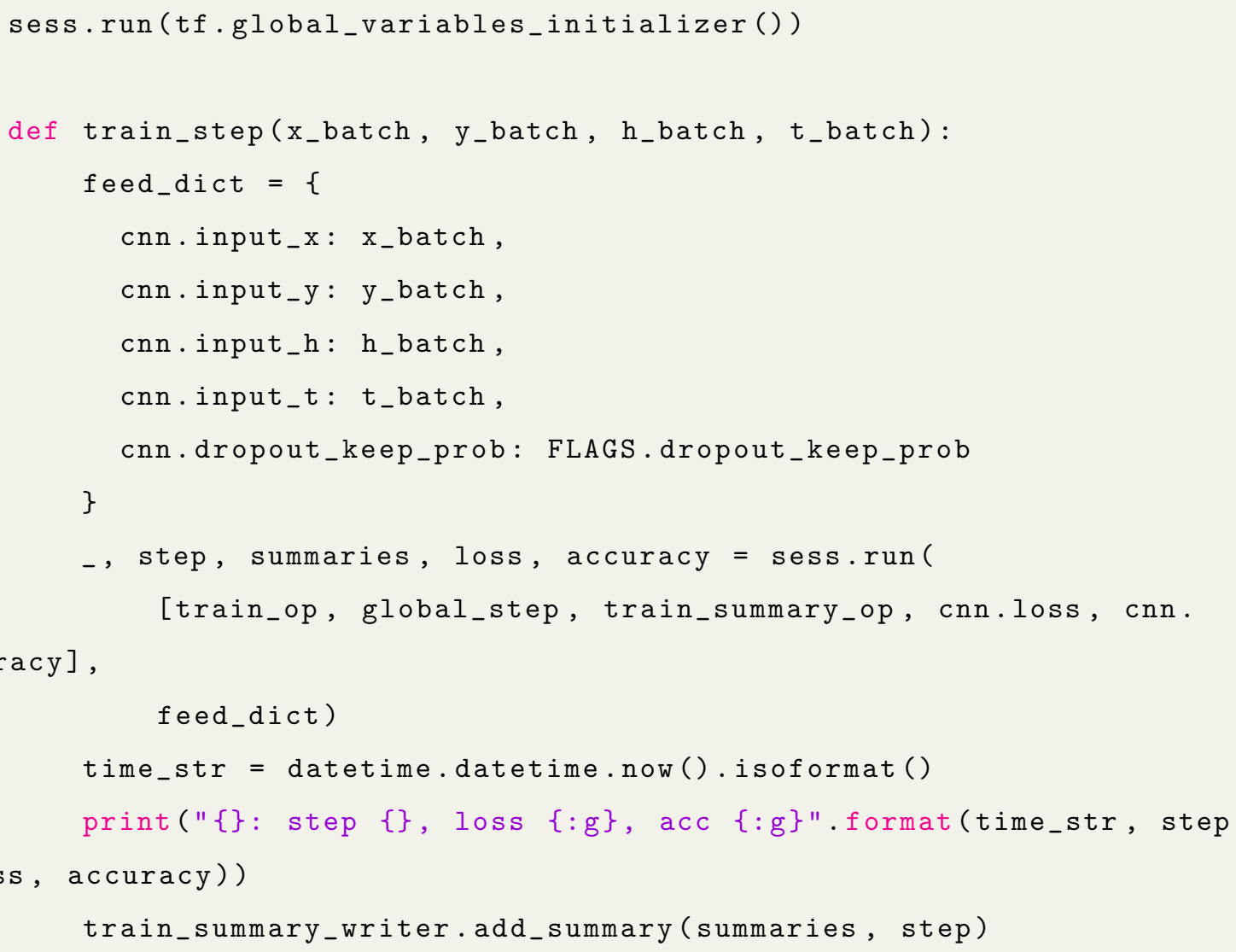

Listing 3.4: Initialize all variables. 
Listing 3.4 show that initialize all variables in each batch.

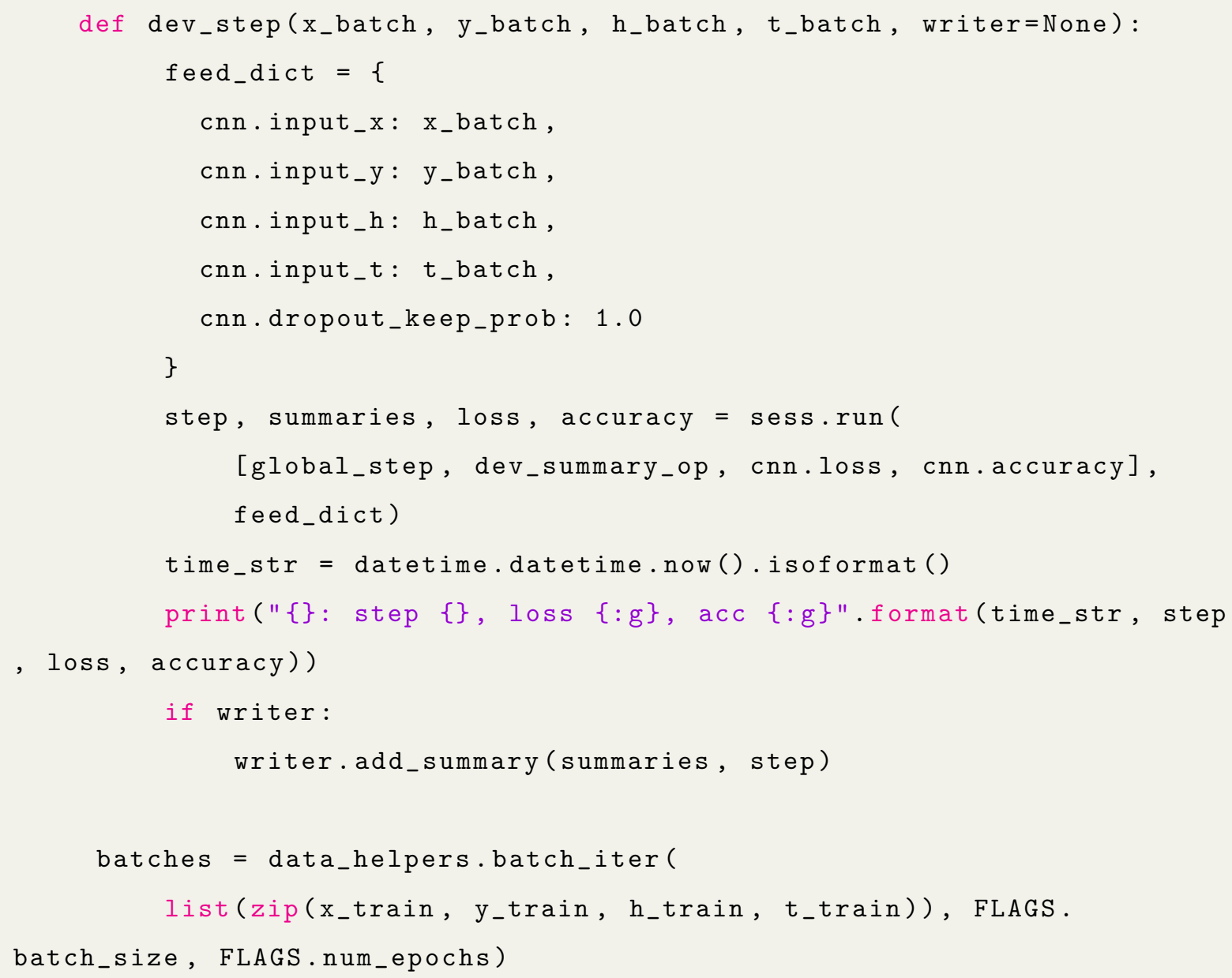

Listing 3.5: Evaluates model and generate batches.

Listing 3.5 show generate batches, which helps to extract feature from each data.

for batch in batches:

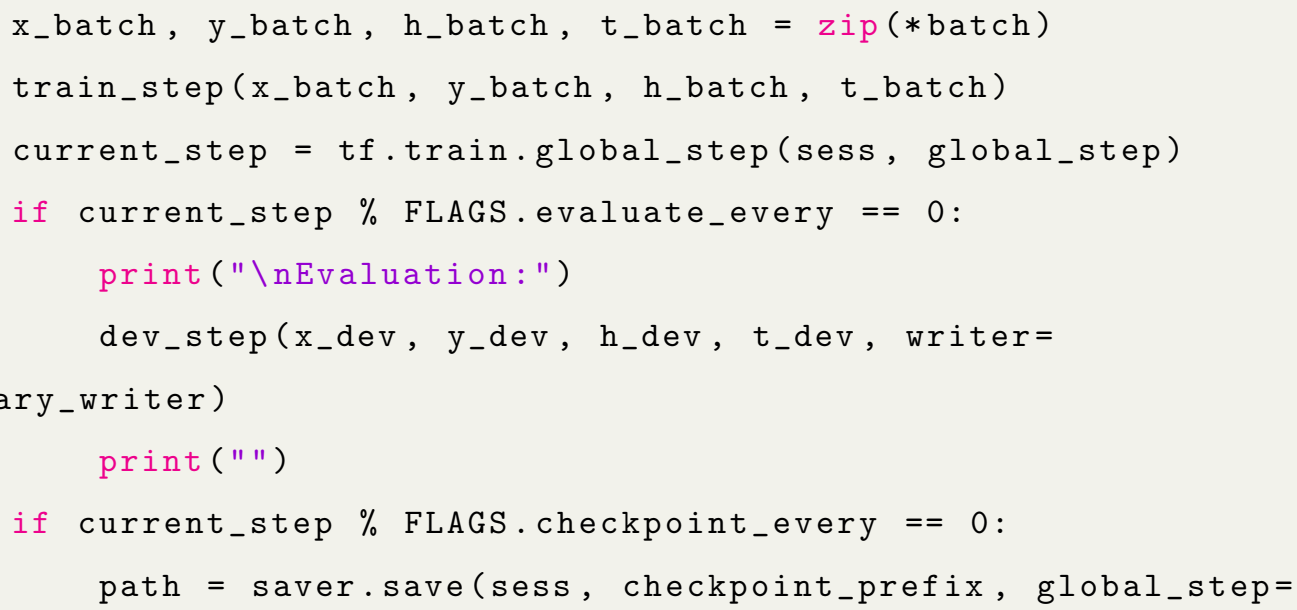




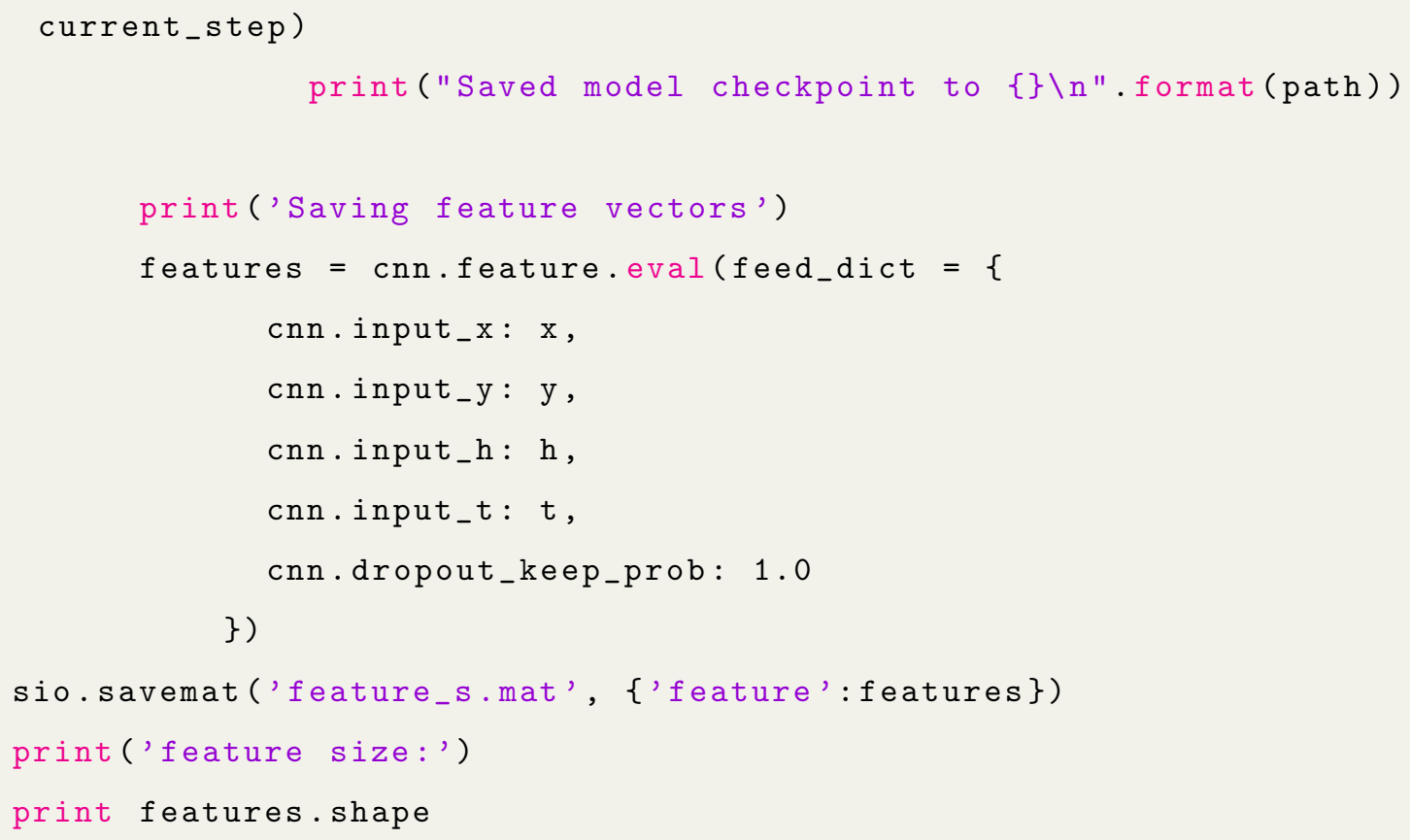

Listing 3.6: Generate feature vector.

Listing 3.6 shows the last step of code of knowledge graph, combined features is generated by knowledge graph and convolutional neural network.

\section{Experimental setting}

Table 3.14: List of machine learning methods.

\begin{tabular}{cc}
\hline Method & Classification \\
\hline & Decision tree (DT) \\
Traditional machine learning & Nogistical regression (LR) \\
& Random forest (RF) \\
& Gradient descent (SGD) \\
& Support vector machine (SVM) \\
\hline Ensemble learning & Adaboost (AB) \\
& Gradient Boosting (GB) \\
\hline
\end{tabular}

This section compares the performance of different models in feature extraction from financial news by expended with full sets of features. The evaluation was intended to obtain evidence that the proposed feature combination model is superior to other feature selection 
Table 3.15: Abbreviations of the results for each algorithm considered.

\begin{tabular}{ccccc}
\hline Algorithm & $\begin{array}{c}\text { Stock data } \\
\text { (SD) }\end{array}$ & $\begin{array}{c}\text { Bag of words } \\
\text { (BOW) }\end{array}$ & $\begin{array}{c}\text { Convolutional } \\
\text { neural } \\
\text { network }\end{array}$ & $\begin{array}{c}\text { Feature } \\
\text { combination } \\
\text { model (FC) }\end{array}$ \\
\hline DT & DT_1 & DT_2 & DT_3 & DT_4 \\
LR & LR_1 & LR_2 & LR_3 & LR_4 \\
NB & NB_1 & NB_2 & NB_3 & NB_4 \\
RF & RF_1 & RF_2 & RF_3 & RF_4 \\
SGD & SGD_1 & SGD_2 & SGD_3 & SGD_4 \\
SVM & SVM_1 & SVM_2 & SVM_3 & SVM_4 \\
AB & AB_1 & AB_2 & AB_3 & B_4 \\
GB & GB_1 & GB_2 & GB_3 & GB_4 \\
\hline
\end{tabular}

models in predicting the stock price movement. As shown in Table 3.14, This study chose linear models, for instance, logistical regression and naive Bayes. Others are non-linear models, for instance, the ensemble learning (random forest, adaBoost and gradient boosting) for the comparison. This study constantly adjusted the parameters in the grid search and selected the optimal parameter value, their parameter value has proven to work well on machine learning method 47,49 .

This study used a computer consisting of an Intel Core i5 processor with four cores running at $2.9 \mathrm{GHz}$ and $8 \mathrm{~GB}$ RAM under a MacOS. This study used the Scikit-learn library in Python in the experiments involving traditional machine learning algorithms and TensorFlow 1.4 in the experiments involving deep learning and the TransE model. The abbreviations used for comparing classification performance are presented in Table 3.15. During testing, 2-fold cross-validation is applied to evaluate the in order to evaluate the stability of the models. And this study compared performance in predicting stock price movement for the next day with a test dataset and evaluated the performance of the models in terms of accuracy and F1-score. 


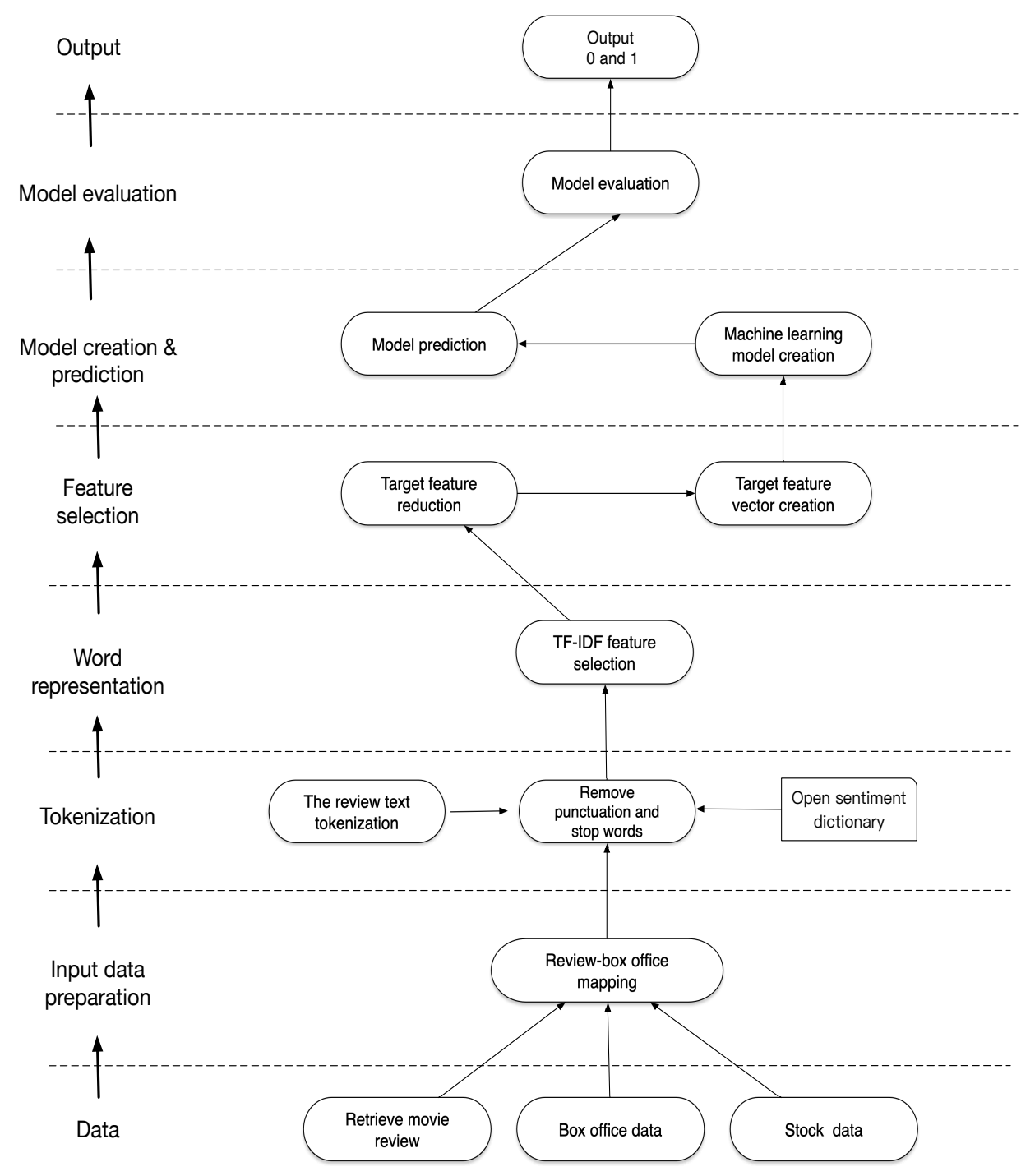

Figure 3.8: The traditional machine learning workflow through TF-IDF feature extraction.

\subsubsection{Application scenario 2}

\section{Machine learning method}

This work selected MLR as a baseline, which evaluated each studio performance, which contained the prediction of the stock market and sentiment classification. Specifically, this work compared between linear regression model as a baseline and classical machine learning.

This section briefly introduces the classical machine learning model using the bag of words approach in feature extraction. Figure 3.8 details this process; it calculated the frequency of the occurrence of terms in the document-term matrix to reduce the dimension for removing 
Table 3.16: Prediction model in classical machine learning.

\begin{tabular}{cc}
\hline Method & Algorithms \\
\hline Baseline & Multiple linear regression (MLR) \\
\hline & Logistical regression (LR) \\
Random forest (RF) \\
Classical machine learning & Extreme Gradient Boosting (XGB) \\
& Gaussian naïve Bayes (GNB) \\
& Support vector machine (SVM) \\
\hline
\end{tabular}

sparse and entering the frequency in the matrix. This matrix presents the term frequencyinverse document frequency (TF-IDF) [193], which is used to score the importance of a word in a document based on how often it appeared in that document and a given collection of documents. If the term appears in the $n_{j}$ of $\mathrm{D}$ review, the TF-IDF weight in the review corpus is computed thus:

$$
D F\left(t_{j}\right)=-\log \left(\frac{n_{j}}{D}\right)
$$

$T F\left(t_{j}\right)$ is the number of times $t_{j}$ appears in the review $i$, then

$$
T F-I D F\left(t_{i j}\right)=T F\left(t_{i j}\right) * I D F\left(t_{j}\right)
$$

The high-frequency words can produce high weighted TF-IDF. Therefore, this method filtered out common words and retained essential words in this work. TF-IDF is often used as a weighting factor in searches of information retrieval, text mining and user modeling. Finally, it created a machine learning model and evaluated the model.

\section{Experiment setting}

This section evaluates the studio performance through the statistical method and the classical machine learning method, which classifies the audience's sentiment and predicts stock price movement. It will also report on their experimental results.

According to the classification task between sentiment analysis and prediction of the stock market, it incorporated classical machine learning into learning processes. In Table 


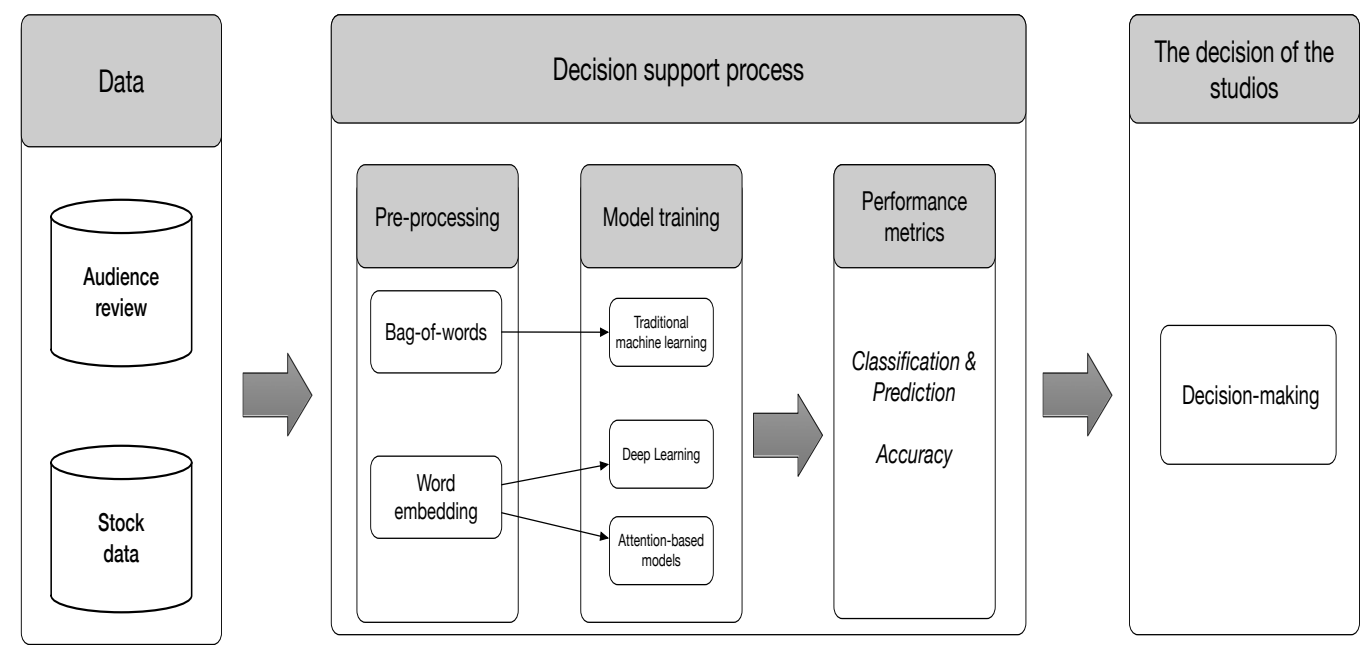

Figure 3.9: Illustrative pipeline for the decision making of studios from narrative materials.

3.16, five algorithms in classical machine learning (e.g. LR, RF, XGB, GNB and SVM) were utilized in the classification task. There were outstanding classification performance and excellent flexibility in these models. The parameters continuously was adjusted the parameters as a grid search in the running system and selected the optimal parameter value in these algorithms.

These algorithms adopted Python with Scikit-learn in this experiment. There was a considerable number of review corpus in the case; this data pre-processing and model training was implemented using the computing system. A five fold cross-validation method was adopted to train the review corpus. All review data were randomly divided into five parts. In each round of data training, it selected one of the five parts as the verification data set; the rest of the data were considered the training data set, and it did not start a new round until all parts were utilized as test data.

This study employed the two metrics for evaluating and comparing the performance in the classification task; these evaluation metrics were accuracy and F1-Score. Accuracy was the most intuitive performance measure, and it was simply a ratio of correctly predicted observation data set to the total observation's data set, which immediately informed us whether a model was being trained correctly and how it might perform generally. 


\subsubsection{Application scenario 3}

This section introduces the methodology for deep learning. It first summarizes the baseline from traditional machine learning and deep learning. Specifically, it compare between traditional machine learning techniques using a bag-of-words approach and deep leaning using word embedding, it is a process that enables an inductive process of classification from the related task. Figure 3.9 illustrates this pipeline to provide decision support of eWOM feature for the studios. It would evaluate the performance of the model through accuracy, sensitivity and specificity on the confusion matrix, and these results help the marketer and manager to make the business decision.

\section{Benchmark from traditional machine learning}

This section briefly introduces the traditional machine learning model with bag-of-words in feature extraction. Figure 3.8 details this process, it calculates the frequency of occurrences of terms in the document term matrix, in order to reduce dimension for removing sparse and put in the frequency in the matrix. This matrix represents the feature for weighting the actual value by frequency-inverse document frequency (TF-IDF), it is a numerical statistic, that intends to reflect how important a word is to a review corpus. TF-IDF is often used as a weighting factor in searches of information retrieval, text mining, and user modeling. It creates different machine learning algorithms for two cases in the studios' performance. In this evolution task, it utilizes traditional machine learning as a baseline.

\section{Hierarchical Two-layer attention network (HTAN)}

Hierarchical two-layers attention network (HTAN) model is a hierarchical network of attention, which consists of the following main components: character level, word level and the classification model. Figure 3.10 shows the architecture of a hierarchical two-layer attention network. This model is inspired by HAN.

1) Character level

Character Encoder. Each words contains $T_{i}$ characters, given a characters with characters $c_{i t}$ with $t \in[0, T]$, it encodes the character to vectors by embedding matrix $W_{c}$, 


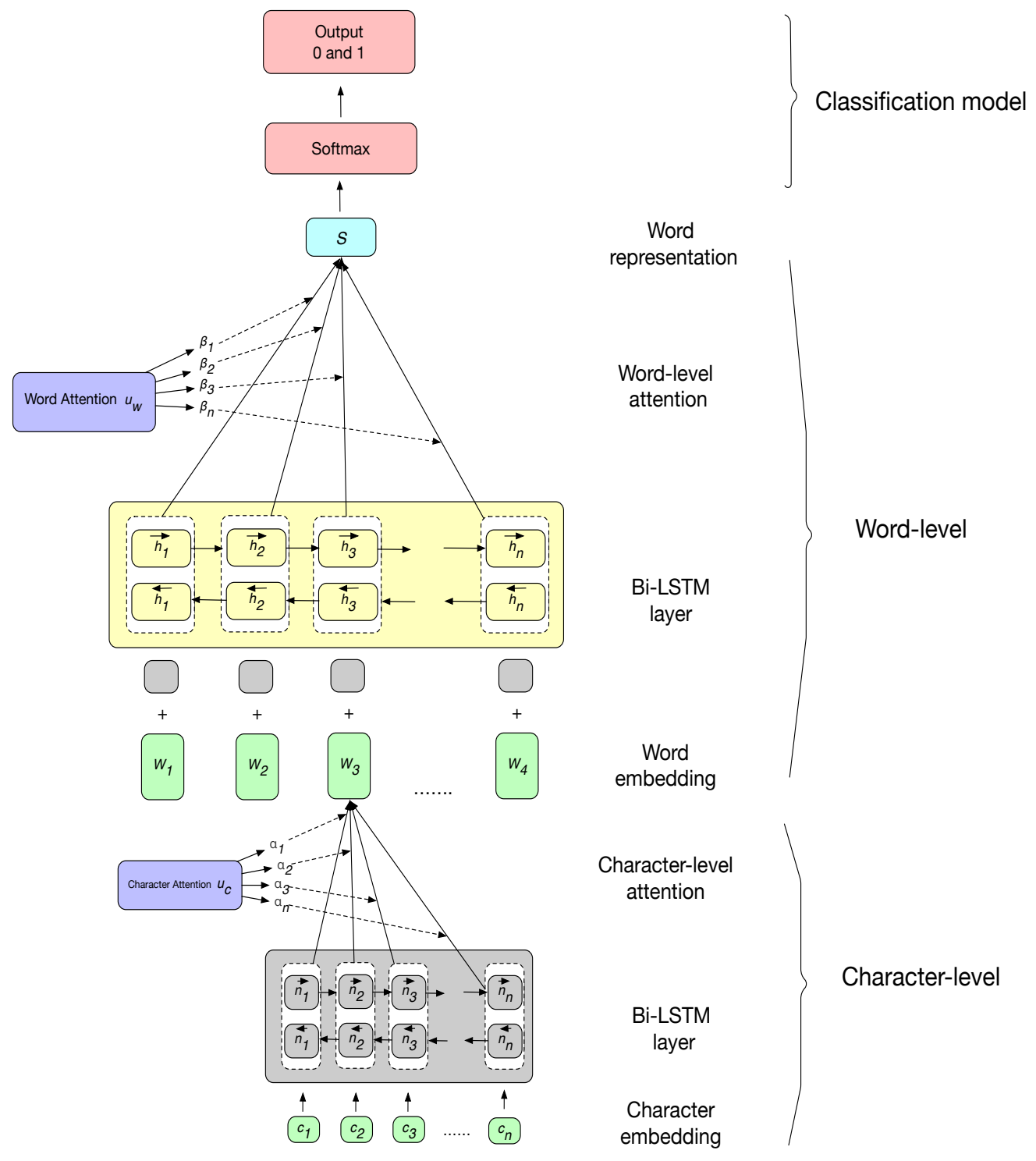

Figure 3.10: A view of HTAN neural network. 
$x_{i j}=W_{c} c_{i j}$. For instance, the word for "Happy", it enters character " $H$ ", " $a$ ", " $p$ ", " $p$ ", " $y$ " in turn. Then it encodes the information of $c_{i t}$ by a bidirectional LSTM, thus it can get its hidden layer representation. The specific calculation method is as follows:

$$
\begin{array}{r}
x_{i t}=W_{c} c_{i t}, t \in[1, T] \\
\overrightarrow{n_{i t}}=\overrightarrow{\operatorname{LSTM}}\left(x_{i t}\right), t \in[1, T] \\
\overleftarrow{n_{i t}}=\overleftarrow{\operatorname{LSTM}}\left(x_{i t}\right), t \in[T, 1] \\
h_{i t}=\left[\overrightarrow{n_{i t}}: \overleftarrow{n_{i t}}\right]
\end{array}
$$

where $\overrightarrow{\operatorname{LSTM}}($.$) and \overleftrightarrow{\operatorname{LSTM}}($.$) denote the forward LSTM and the backward LSTM,$ respectively. $\left[\overrightarrow{n_{i t}}: \overleftarrow{n_{i t}}\right]$ denotes a concatenating operation on $\overrightarrow{n_{i t}}$ and $\overleftarrow{n_{i t}}$

Character attention. The words are composed of characters that represent different importance, and the contribution for each character to indicate the overall sentiment polarity may vary from each other. Here is used self-attention in this attention network. Hence, this architecture introduced HAN to distinguish different characters in a word.

$$
\begin{array}{r}
u_{i t}=\tanh \left(W_{w} h_{i t}\right) \\
\beta_{i}=\frac{\exp \left(u_{i t}\right)}{\sum_{t} \exp \left(u_{i t}\right)} \\
\mathbf{s}_{\mathbf{i}}=\sum_{i t} \beta_{i} h_{i t}
\end{array}
$$

where $a_{i j}$ consider audience review, the importance of the $j-t h$ character in the word construct the representation of the entire word $S_{i}$. $W_{c} \in \mathbb{R}^{v}$ denotes the parameter for alignment function to calculate the match between $h_{i t}$ itself. It assesses the importance of the character that the similarity $u_{i t}$ between the character level context vector $u_{c}, \alpha_{i t}$ is normalized importance weight by the softmax function. As briefly described above, the reason it leverage the character level attention is that find character-level emotional features and understand fine-grained eWOM features in audience sentiment. 
2) Word level

Word encoder. After obtaining the character vector $S_{i}$, this layer also adopts bidirectional LSTM to encode word and gain a hidden layer representation $h_{i}$ at the end of the time step. It gains the representation of word i through the concatenating of $\overrightarrow{h_{i}}$ and $\overleftarrow{h_{i}}$.

$$
\begin{array}{r}
\overrightarrow{h_{i}}=\overrightarrow{\operatorname{LSTM}}\left(x_{i}\right), i \in[1, T] \\
\overleftarrow{h_{i}}=\overleftarrow{\operatorname{LSTM}}\left(x_{i}\right), i \in[T, 1] \\
h_{i t}=\left[\overrightarrow{h_{i t}}: \overleftarrow{n_{i t}}\right]
\end{array}
$$

Word attention. It then uses a word-level context vector through attention mechanism to decode the word level representation among the entire sentence. This attention layer assesses the importance of each word. This denotes:

$$
\begin{array}{r}
u_{i}=\tanh \left(W_{w} h_{i}\right) \\
\beta_{i}=\frac{\exp \left(u_{i}\right)}{\sum_{t} \exp \left(u_{i}\right)} \\
\mathbf{s}=\sum_{i} \beta_{i} h_{i}
\end{array}
$$

where $\mathrm{s}$ is the parameter for each word in the softmax layer, the weight sum $\mathrm{s}$ is computed by $s$, it can combine character-level attention with word-level attention in the review information. The effects of this attention layer are similar to character level.

3) Classification model

After calculating the comment text vector $S$, it calculates the probability distribution for each category $p$.

$$
p=\operatorname{softmax}\left(W_{s} v+b_{v}\right)
$$


where $W_{s}$ and b present the weight parameters and offsets in the softmax layer. This outputs two classes (0 and 1); these denote positive or increase and negative or decrease, which examine the availability of the attention model in studios. Finally, the cross-entropy is used as the training loss function.

$$
L=-\sum_{r} \log p_{r j}
$$

where $j$ is the label in audience review $r$.

\section{Python code of HTAN}

This code explains the technical details of applying deep learning with attention mechanism to classification with the company performance. Software requirements pandas 0.20 .3 , tensorflow 1.6.0, keras 2.0.8 and numpy 1.14.0. The detailed code of the model is as follows:

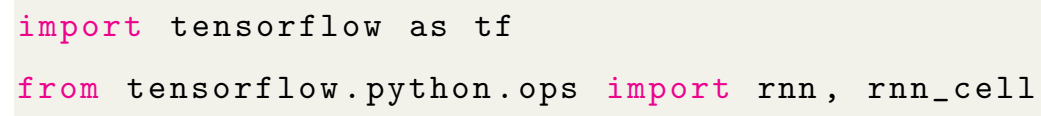

Listing 3.7: Package input

Listing 3.7 shows the each pakege input.

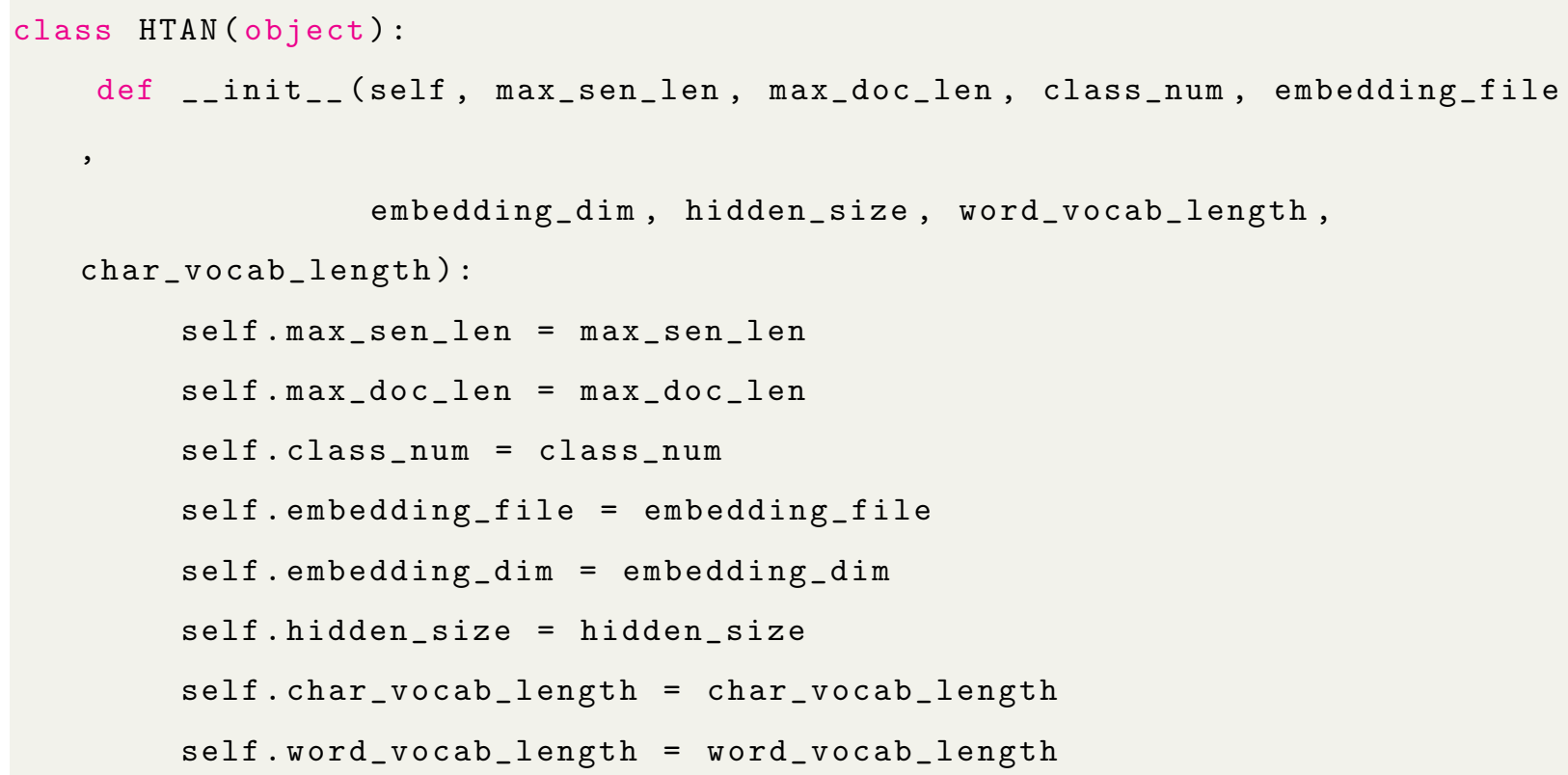




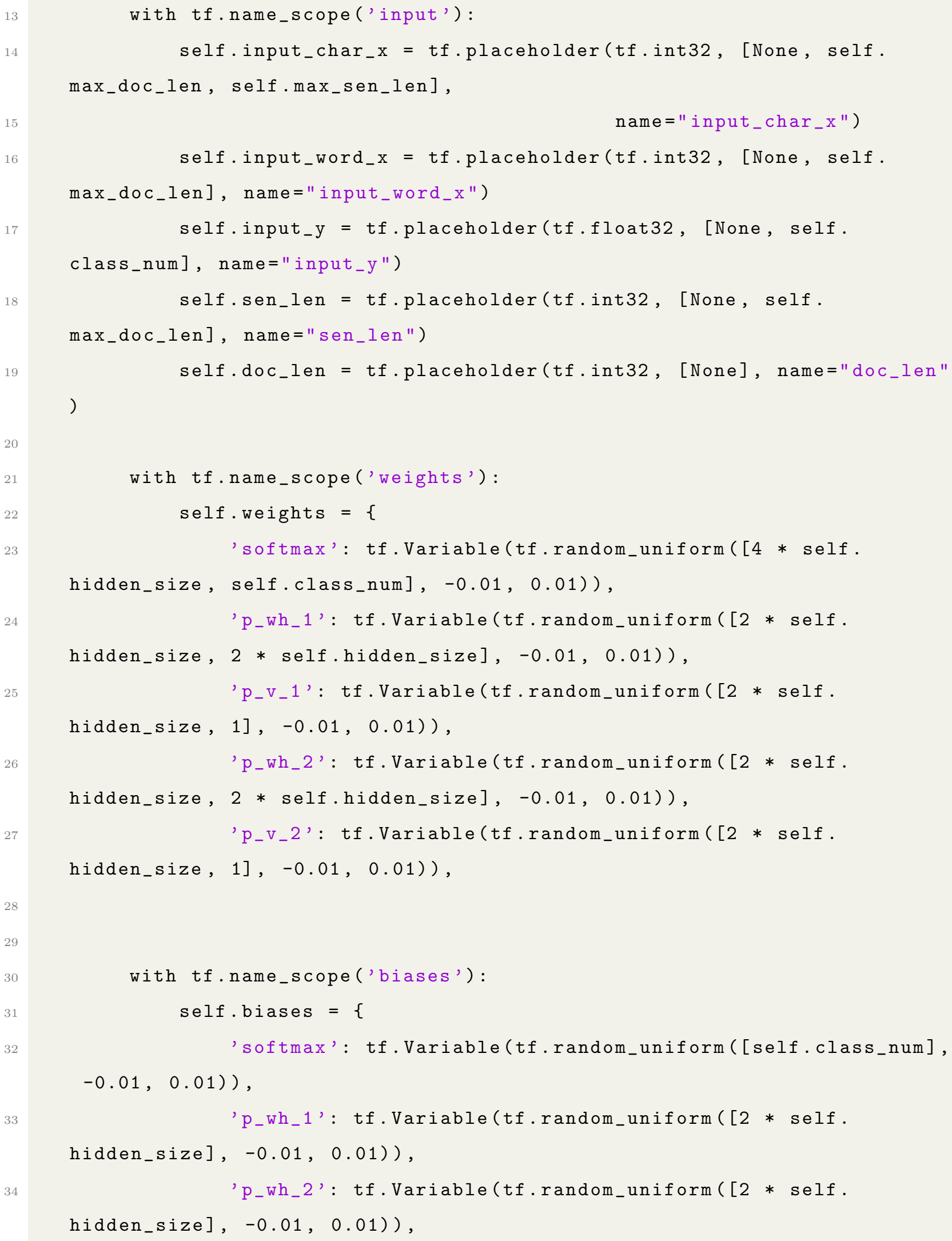

with tf.name_scope('input'):

self.input_char_x = tf.placeholder(tf.int32, [None, self. max_doc_len, self.max_sen_len],

name $=$ " input_char_ $\mathrm{x} "$ )

self.input_word_x = tf.placeholder(tf.int32, [None, self.

max_doc_len], name="input_word_x")

self.input_y = tf.placeholder(tf.float 32, [None, self.

class_num], name="input_y")

self.sen_len = tf.placeholder(tf.int 32, [None, self.

max_doc_len], name="sen_len")

self.doc_len = tf.placeholder(tf.int32, [None], name="doc_len" )

with tf.name_scope ('weights'):

self. weights $=\{$

'softmax': tf.Variable(tf.random_uniform ( $[4 *$ self .

hidden_size, self.class_num], -0.01, 0.01)),

'p_wh_1': tf.Variable(tf.random_uniform ( $[2 *$ self .

hidden_size, $2 *$ self.hidden_size], $-0.01,0.01)$ ),

'p_v_1': tf.Variable(tf.random_uniform( [2 * self .

hidden_size, 1], $-0.01,0.01)$ ),

'p_wh_2': tf.Variable(tf.random_uniform ([2 * self.

hidden_size, $2 *$ self.hidden_size], $-0.01,0.01)$ ),

'p_v_2': tf.Variable(tf.random_uniform( $[2 *$ self.

hidden_size, 1], $-0.01,0.01)$ ),

with tf.name_scope('biases'):

self.biases $=\{$

'softmax': tf.Variable(tf.random_uniform([self.class_num], $-0.01,0.01))$,

'p_wh_1': tf.Variable(tf.random_uniform ( $[2 *$ self.

hidden_size], $-0.01,0.01)$ ),

'p_wh_2': tf.Variable(tf.random_uniform ( $[2 *$ self .

hidden_size ], $-0.01,0.01)$ ), 


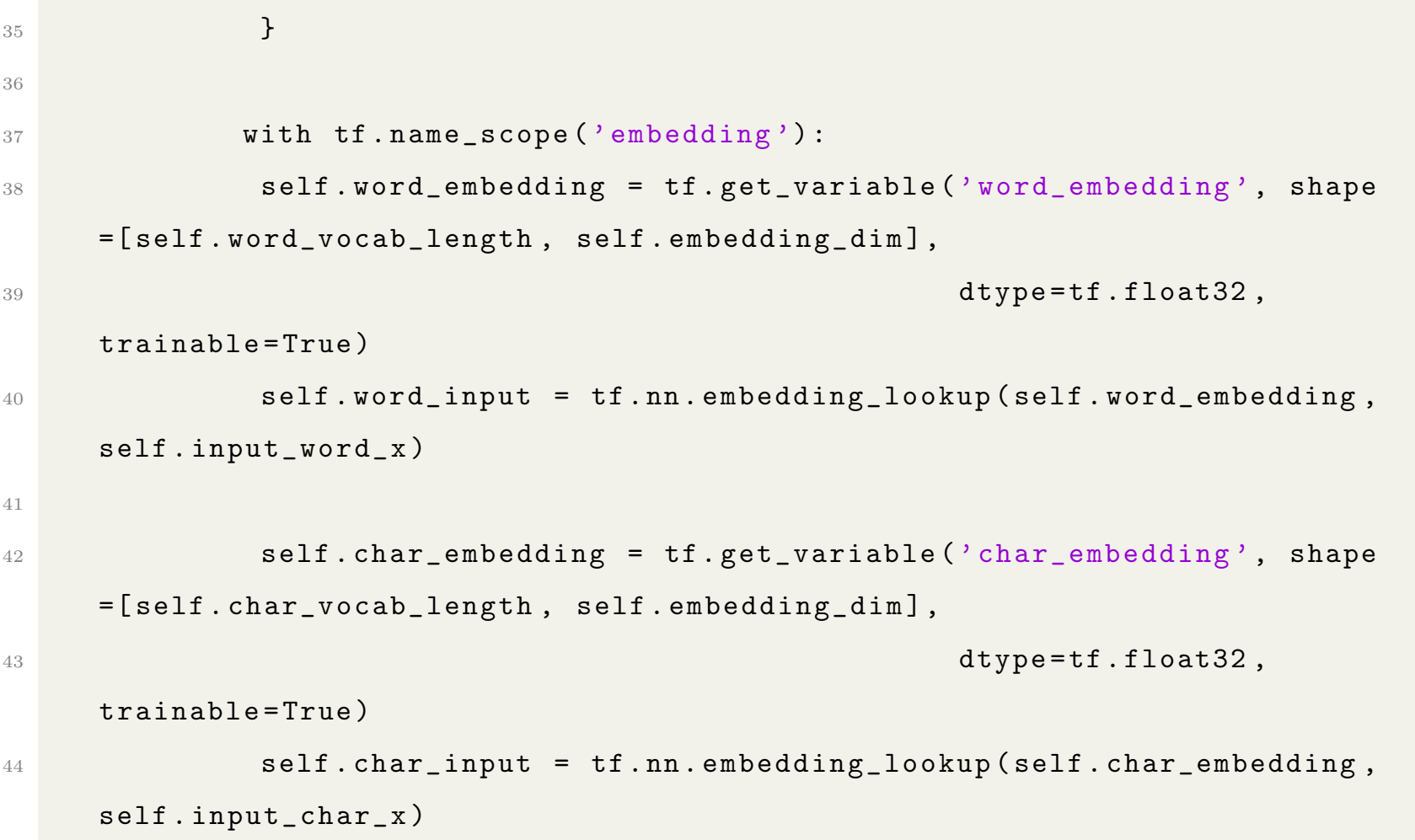

Listing 3.8: Definition of the main structure of HTAN

Listing 3.8 defined the main structure of HTAN, which contains input, weights, biases and embedding.

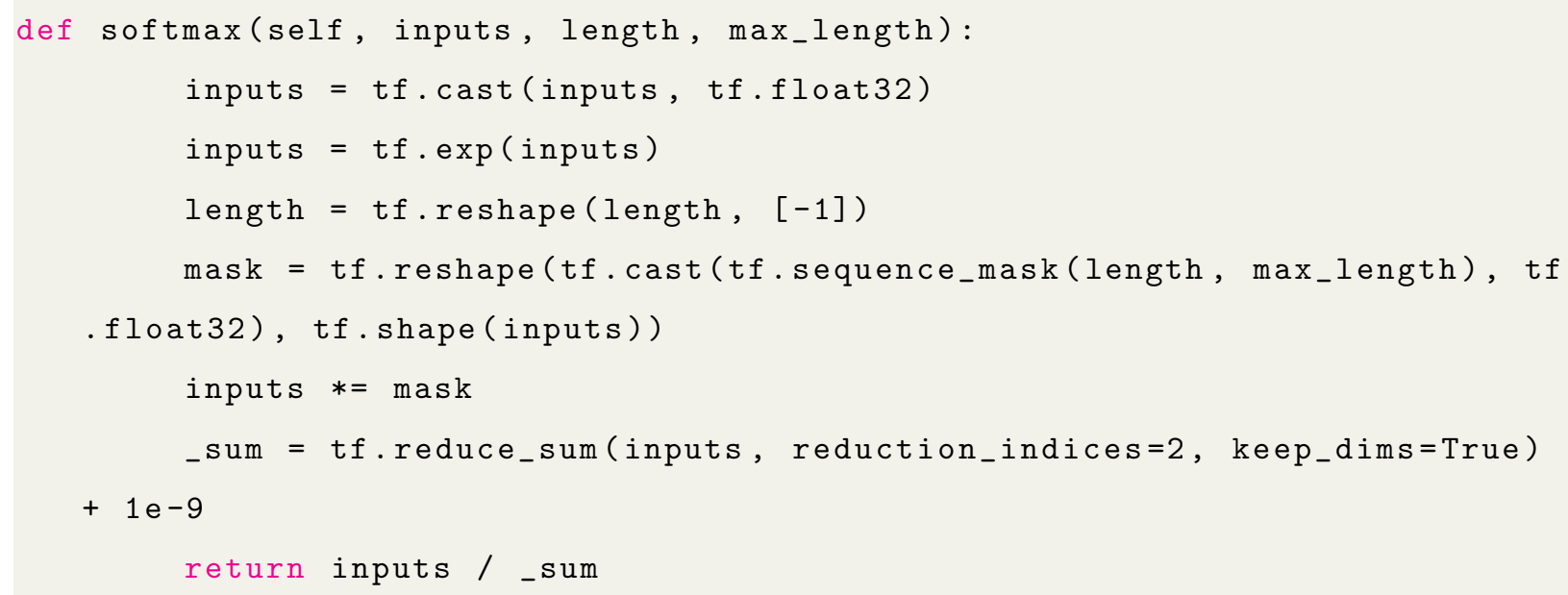

Listing 3.9: Definition of parameters of softmax

Listing 3.9 defines as the parameters of softmax.

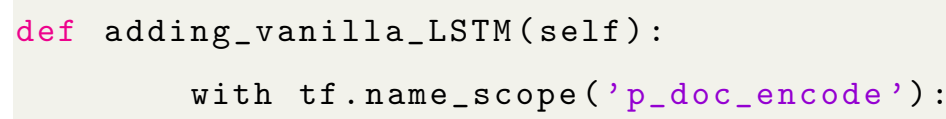


Chapter 3. Materials and frameworks design

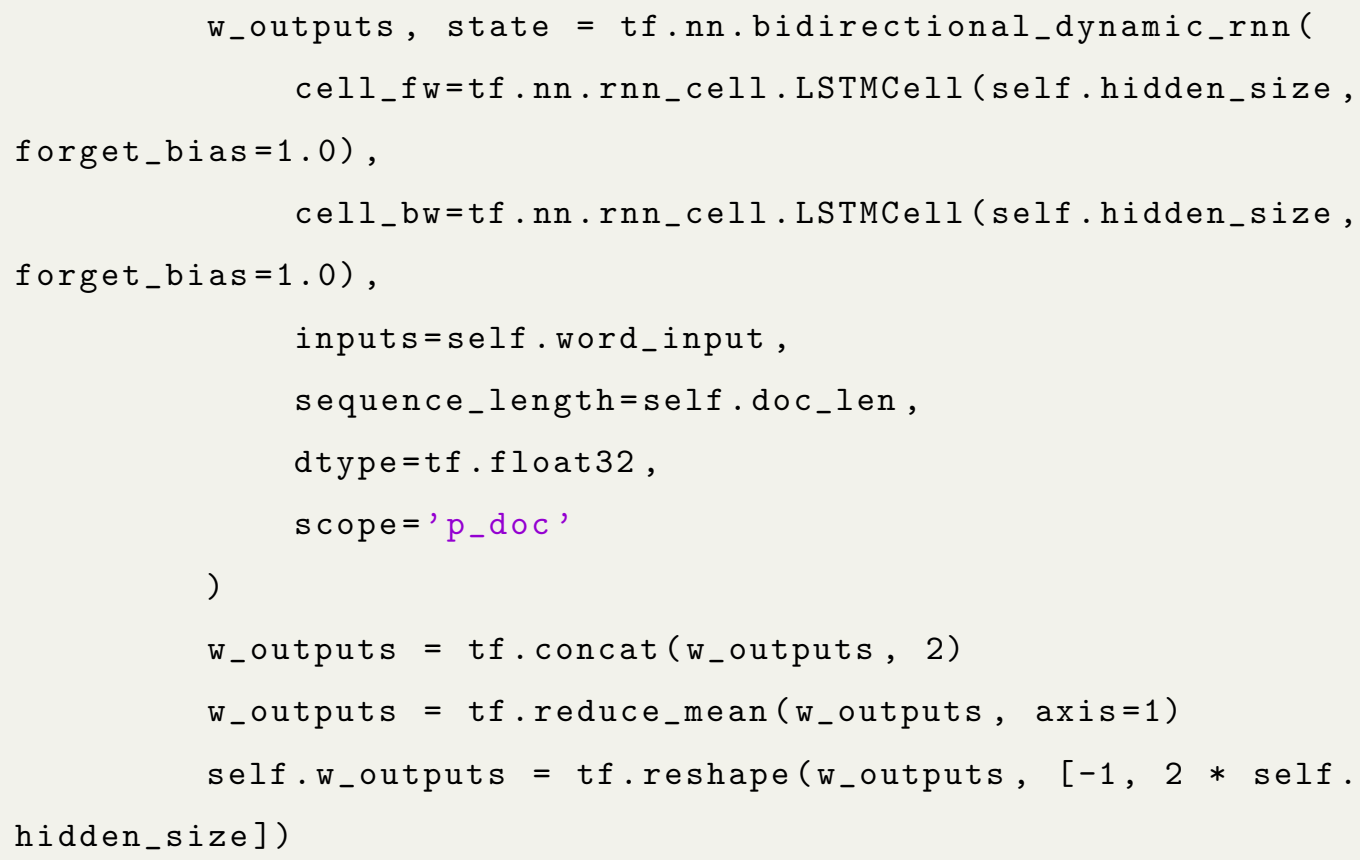

Listing 3.10: Definition of parameters of bi-LSTM

Listing 3.10 defines parameters of bi-LSTM, which help with feature extraction.

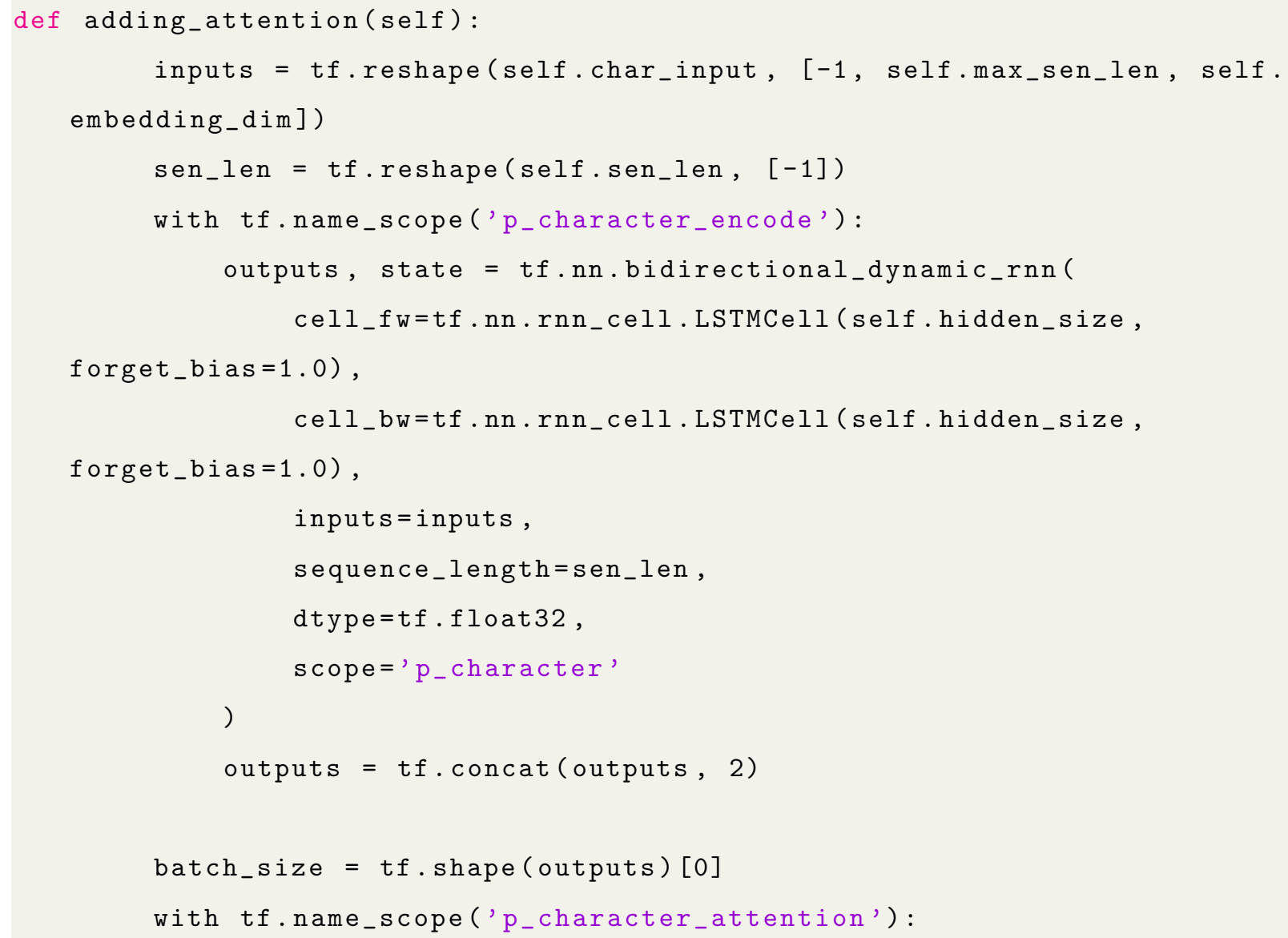


output $=$ tf.reshape (outputs, $[-1,2 *$ self.hidden_size $]$ )

$\mathrm{u}=$ tf.matmul (output, self.weights ['p_wh_1']) + self.biases [' $\mathrm{p}_{-} \mathrm{wh}_{-}{ }^{\prime}$ ']

$u=t f . r e s h a p e(u,[-1$, self.max_doc_len $*$ self.max sen_len, 2 * self.hidden_size])

$\mathrm{u}=\mathrm{tf} \cdot \tanh (\mathrm{u})$

$u=t f . r e s h a p e(u,[-1,2 *$ self.hidden_size $])$

alpha $=$ tf.reshape(tf.matmul(u, self.weights ['p_v_1'] ),

[batch_size, 1, self.max_sen_len])

alpha $=$ self.softmax (alpha, self.sen_len, self.max_sen_len)

outputs = tf.matmul(alpha, outputs)

outputs = tf.reshape(outputs, $[-1$, self.max_doc_len, $2 *$ self. hidden_size])

with tf.name_scope('p_word_encode'):

outputs, state = tf.nn.bidirectional_dynamic_rnn(

cell_fw=tf.nn.rnn_cell. LSTMCell (self .hidden_size ,

forget_bias=1.0),

cell_bw=tf.nn.rnn_cell. LSTMCell(self.hidden_size,

forget_bias=1.0),

inputs =outputs,

sequence_length=self.doc_len,

dtype=tf.float 32 ,

scope $=$ 'p_character'

)

outputs = tf. concat (outputs, 2)

batch_size $=$ tf.shape(outputs) $[0]$

with tf.name_scope('p_word_attention'):

output = tf.reshape(outputs, $[-1,2 *$ self.hidden_size $]$ )

$u=$ tf.matmul(output, self.weights ['p_wh_2']) + self.biases [' $\mathrm{p}_{-}$wh_ $2^{\prime}$ ]

$u=$ tf.reshape $(u,[-1$, self.max_doc_len, $2 *$ self.hidden_size ])

$\mathrm{u}=\mathrm{tf} \cdot \tanh (\mathrm{u})$ 
Chapter 3. Materials and frameworks design

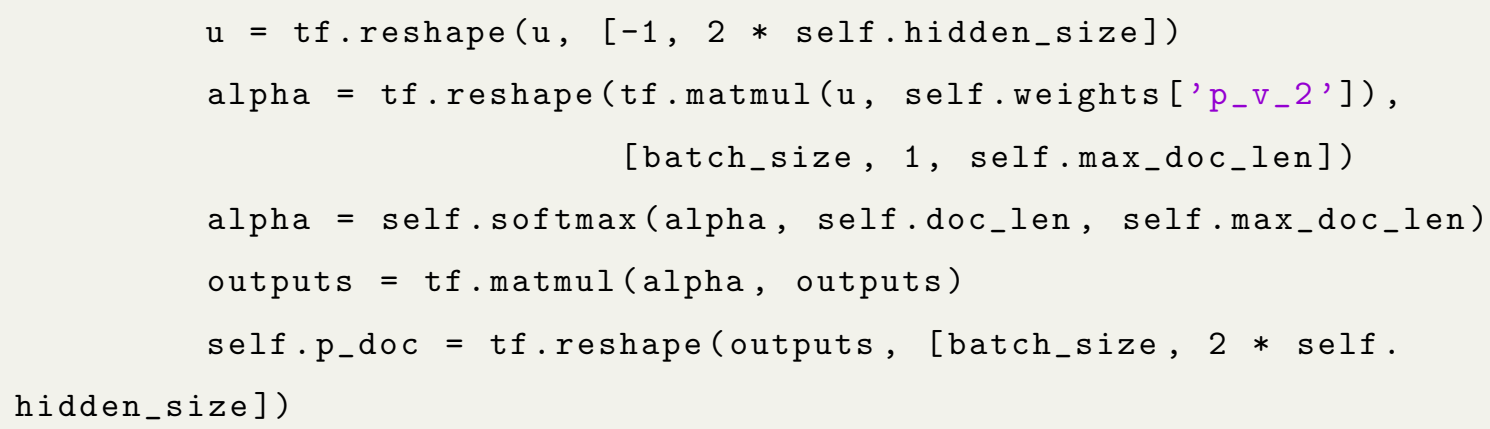

Listing 3.11: Attention mechanism in character level and word level

Listing 3.11 shows attention mechanism in character level and word level, and it mainly extract features from both characters and words.

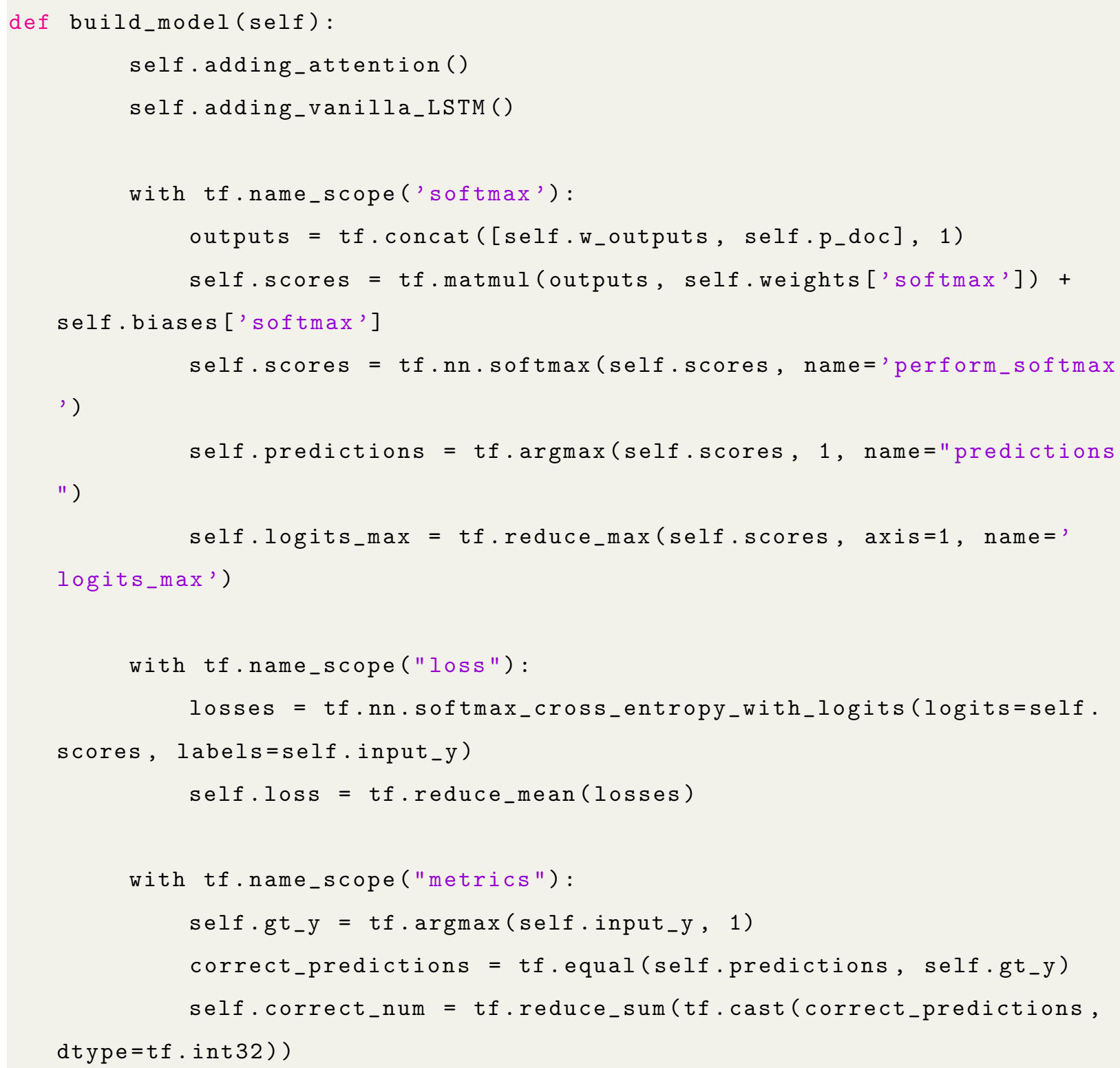


Listing 3.12: Definition of model building

Listing 3.12 builds attention-based model for HTAN.

\section{Hierarchical convoluted attention network}

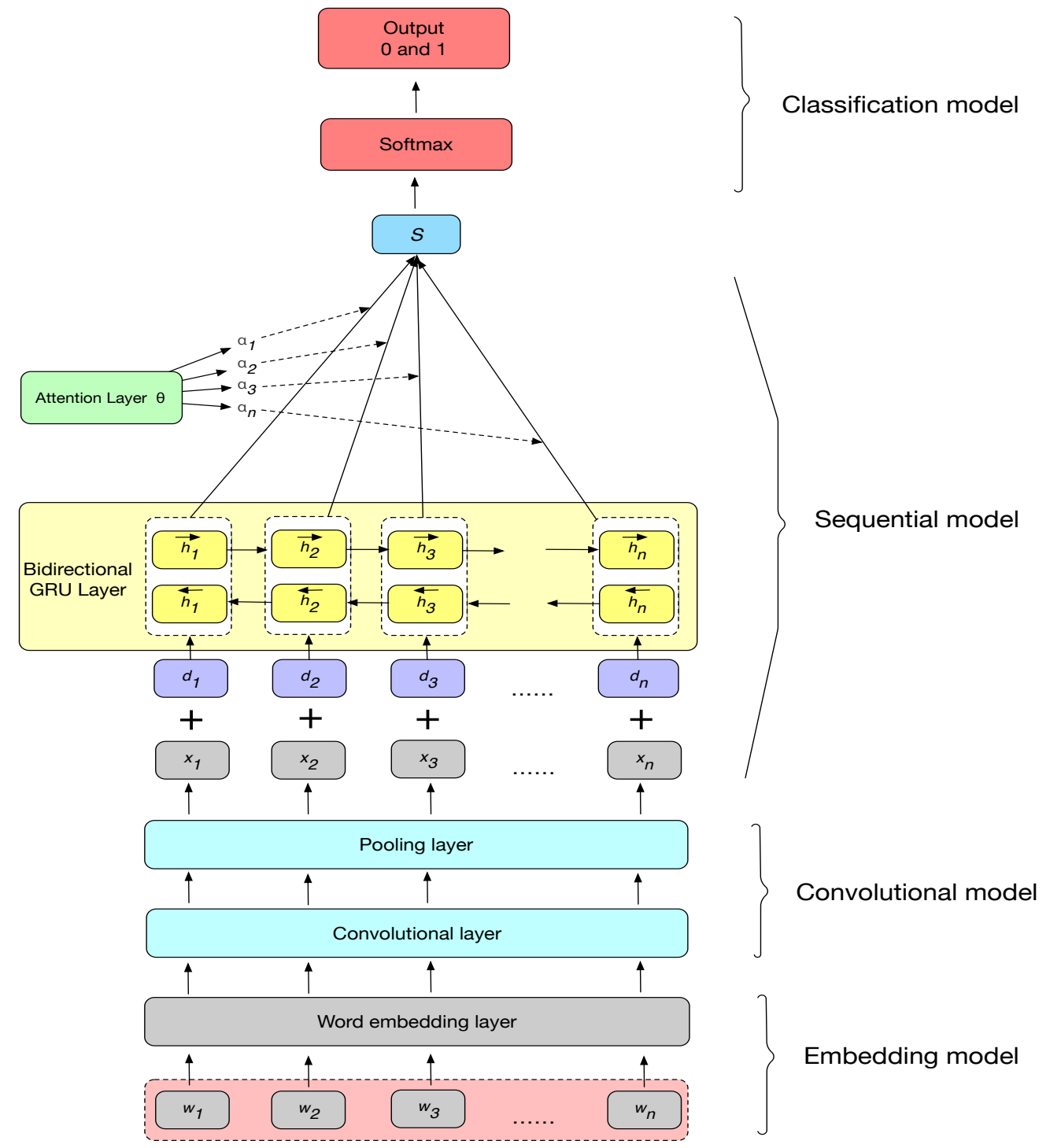

Figure 3.11: A view of HCAN neural network.

Figure 3.11 shows the proposed model should pay more attention to the importance of 
review. This framework should highlight the important sentiment characteristics of each review.

In the proposed architecture as HCAN. First, it pre-processings each review as input the review corpus sequence on the word embedding layer, which encodes each review to a representation vector. In this way, these vectors fill into the convolutional layer and polling layer, which generates the convolutional vector in each word. Second, these the convolutional vectors are encoded by the bi-gated recurrent units (GRU). Finally, the attention mechanism layer assigns the attention value to each important word and calculates a weighted word vector, which represents the important feature for the entire context. The detail of the architecture is below:

1) Embedding model

Word embedding is that the word in a high-dimensional space map into a continuous vector space with a lower dimension. This embedding layer converts each input word sequence into a corresponding embedding vector by consulting lookup table. Given the audience review $\mathrm{r}$ with $\mathrm{n}$ words containing $w=\left\{w_{1}, w_{2}, w_{3} \ldots, w_{n}\right\}$, it calculates each embedding vector through word embedding layer. Each word in w is mapped 100-dimensional vector, which looked up in pre-trained word embedding by Glove [55] for each word to represent the word embedding $w_{i}=\left\{w_{1 i}, w_{2 i}, w_{3 i} \ldots, w_{n i}\right\}$. Lately, these words vector input the convolutional layer.

2) Convolutional model

Convolutional neural network $(\mathrm{CNN})$ is a combination of convolutional layer and pooling layer. The output of each layer is the input to the next layer. As a feature extraction layer, the convolutional layer extracts word features through a filter, generated a feature map through a convolution kernel function. Pooling layer belongs to the feature mapping layer, which samples the feature word generated by the convolutional layer and outputs local optimal features. In this work, it introduced the feature representation of the convolution model, which is designed based on Text-CNN 194.

$$
x_{1: n}=x_{1} \oplus x_{1} \oplus x_{2} \oplus \ldots \oplus x_{n}
$$


in which $\oplus$ denotes the concatention. The N-gram feature $c_{i}$ represents the $i-t h$ eigenvalue in the word embedding, which is window size of $X_{i: i+h-1}$, it represents:

$$
c_{i}=\sigma\left(w X_{i: i+h-1}+b\right)
$$

where $\sigma($.$) is convolution kernel function, \mathbf{w} \in \mathbb{R}_{n * k}$ is a filter, $h=5$ is the size of the sliding window, and $\mathrm{b}$ is the offset value. $X_{i: i+h-1}$ means the feature matrix of word embedding from the $i-t h$ to the $i+h-1$. Its length is 100. Hence, a feature map $c$ denotes:

$$
c=\left[c_{1}, c_{2}, \ldots, c_{n-h+1}\right]
$$

The characteristic table $\mathrm{c}$ is taken out as a feature of the next layer through a time maximization pooling operation.

$$
c_{\max }=\left[\begin{array}{c}
\max \left(c_{1}\right) \\
\cdots \\
\max \left(c_{d}\right)
\end{array}\right]
$$

Then, the max-pooled features are passed in next layer, its length is also 100.

3) Sequential mode

A document $\mathrm{d}$ with $\mathrm{n}$ words $d_{i}=\left\{d_{1}, d_{2}, d_{3}, \ldots, d_{n}\right\}$ merges word feature by CNN, and this new word vector in the same position as $w_{i}$. which extracts crucial word feature in the next attention layer. The review information in the past or future by a bidirectional GRU, which calculates their potential feature vectors. It concatenates potential vectors from both directions for constructing a bidirectional encoded vector $h_{i s}$, it follows as: 


$$
\begin{array}{r}
\overrightarrow{h_{i s}}=\overrightarrow{G R U}\left(x_{i s}: w_{i s}\right), t \in[1, L] \\
\overleftarrow{h_{i s}}=\overleftarrow{G R U}\left(x_{i s}: w_{i s}\right), t \in[L, 1] \\
h_{i s}=\left[\overrightarrow{h_{i s}}: \overleftarrow{n_{i s}}\right]
\end{array}
$$

In summary, it employs the attention mechanism for finding the most contributing of the sentence in the review corpus. Moreover, $h_{i s}$ input a single layer perceptron for getting the result $u_{i s}$, which represents:

$$
u_{i s}=\tanh \left(W_{w} h_{i s}\right)
$$

To measure the importance of each review, it adopts $\theta$ to express the similarity of a randomly initialized context vector $u_{i}$, then it obtains a normalized attention weight matrix $\alpha_{i}$ through softmax operation, this learning process denotes as:

$$
\alpha_{i}=\frac{\exp \left(\theta_{i} u_{i s}\right)}{\sum_{j} \exp \left(\theta_{i} u_{i s}\right)}
$$

Furthermore, the weight value ${ }_{i}$ continues to compute the sum weight $S$, its size is 100 and it shows that:

$$
S=\sum_{i} \alpha_{i} h_{i s}
$$

This approach combines sequential review by attention model, which facilities to apply in the motion picture industry.

4) Classification model

The softmax operation on two cases study application. The output the probability distribution $p$ over two categories, 


$$
p=(y=j \mid D)=\operatorname{softmax}\left(W_{s} S+b_{s}\right)
$$

where $W_{s}$ is a weight matrix, $b_{s}$ is bias vector that to be learned in the softmax layer. And $j$ is the label of classification ( 0 and 1$)$, these denote positive or increase and negative or decrease, respectively.

\section{Python code of HCAN}

These codes detail the proposed HCAN model, software requirements is pandas 0.20.3, tensorflow 1.6, keras 2.0.8 and numpy 1.14. The detailed code of the model is as follows:

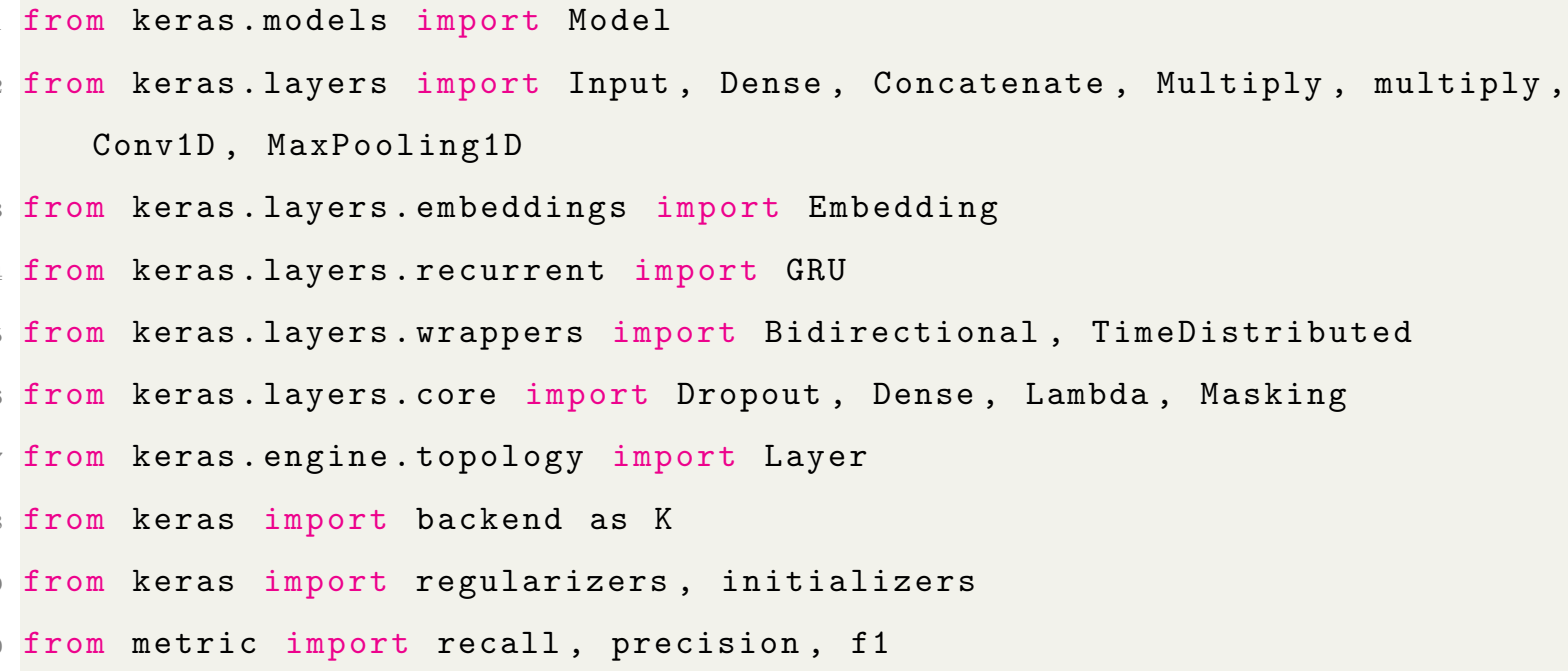

Listing 3.13: Input package

Listing 3.13 shows some package input. In particular, it emphasized the powerful advantages of the Keras package

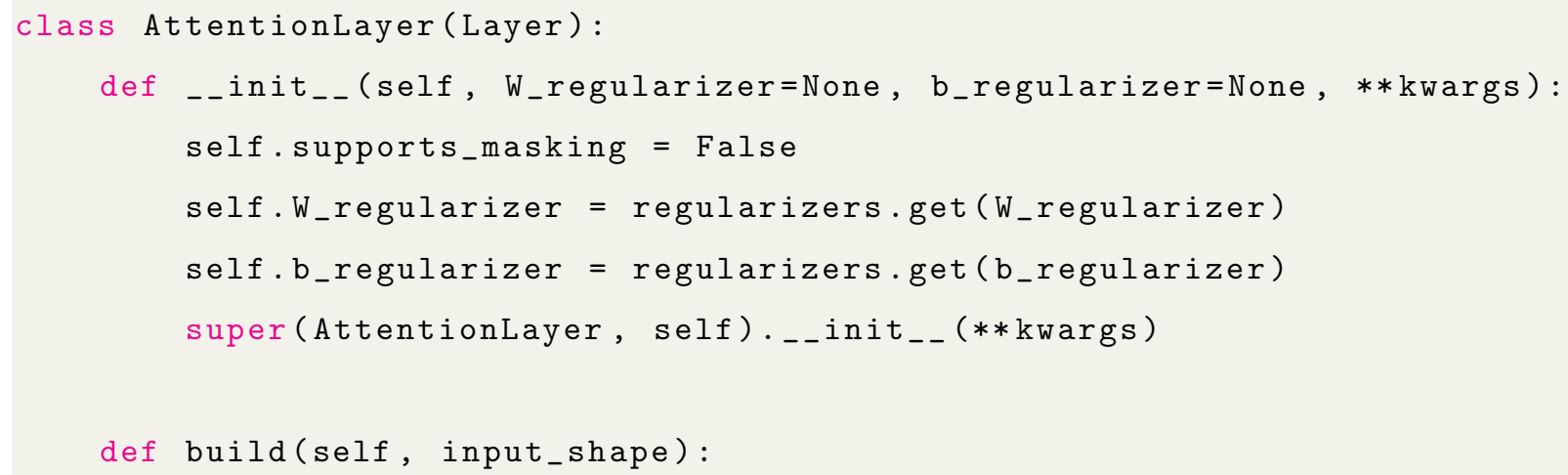


Chapter 3. Materials and frameworks design

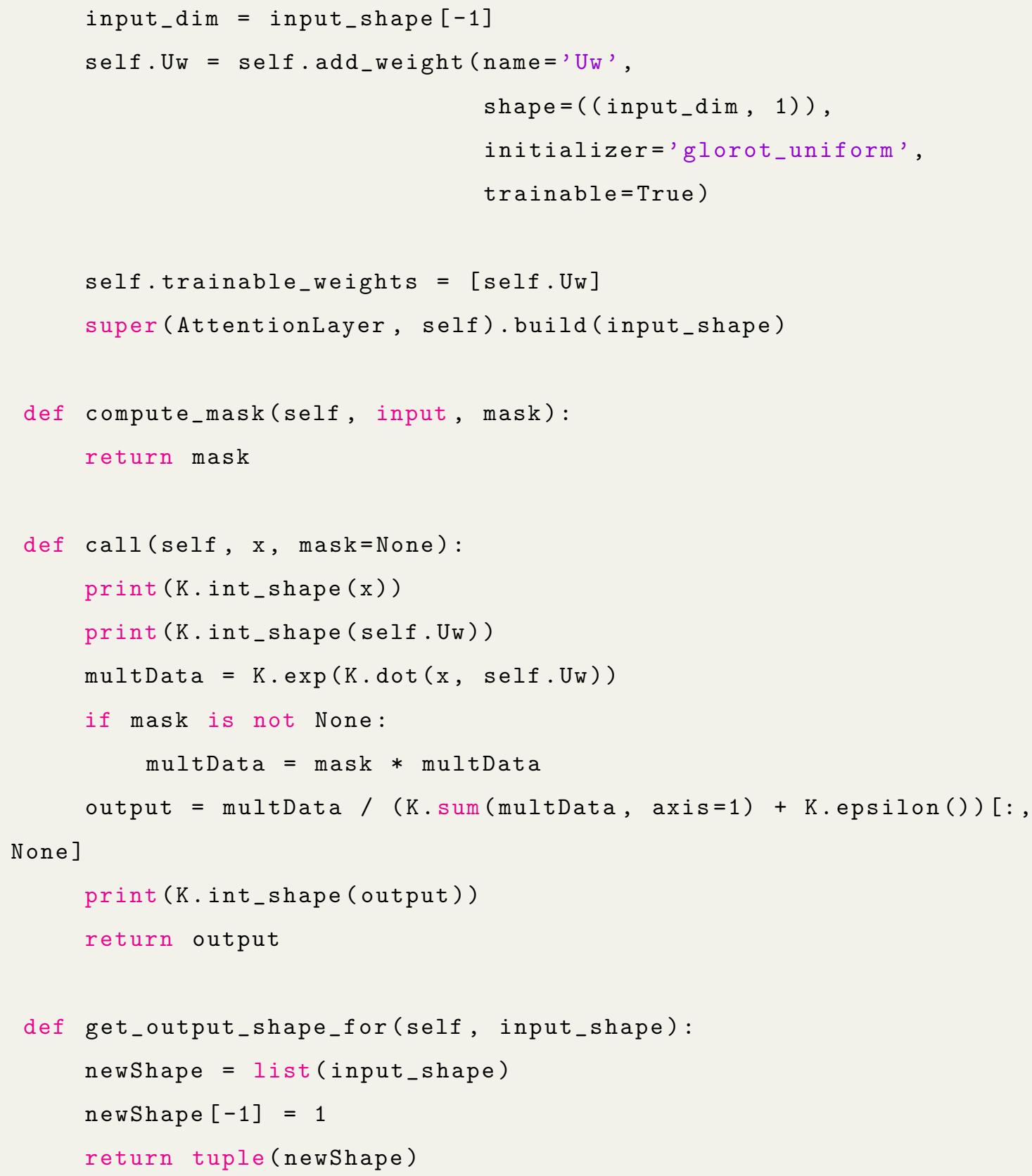

Listing 3.14: Attention layer.

Listing 3.14 defines the attention layer, which averages the weighted of word embedding.

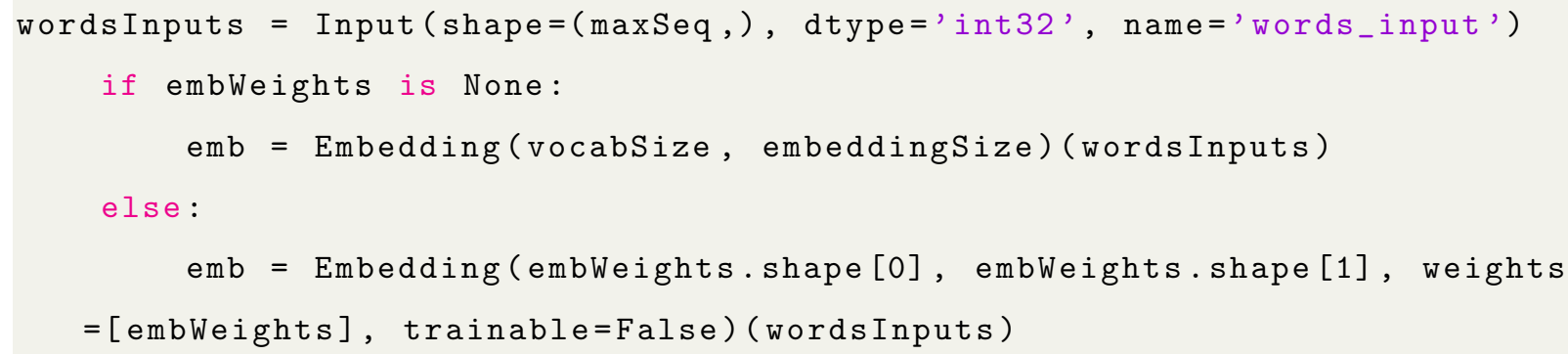


if dropWordEmb $\quad !=0.0$ :

emb $=$ Dropout (dropWordEmb) (emb)

Listing 3.15: Definition parameters of word embedding layer.

Listing 3.15 shows the parameters of the word embedding layer.

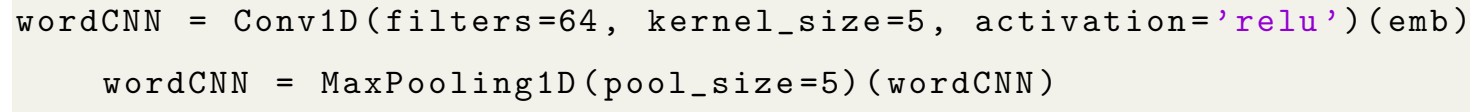

Listing 3.16: Definition parameters of CNN in word embedding

Listing 3.16 shows the parameters of CNN in word embedding, which extracts features from each word of each sentence.

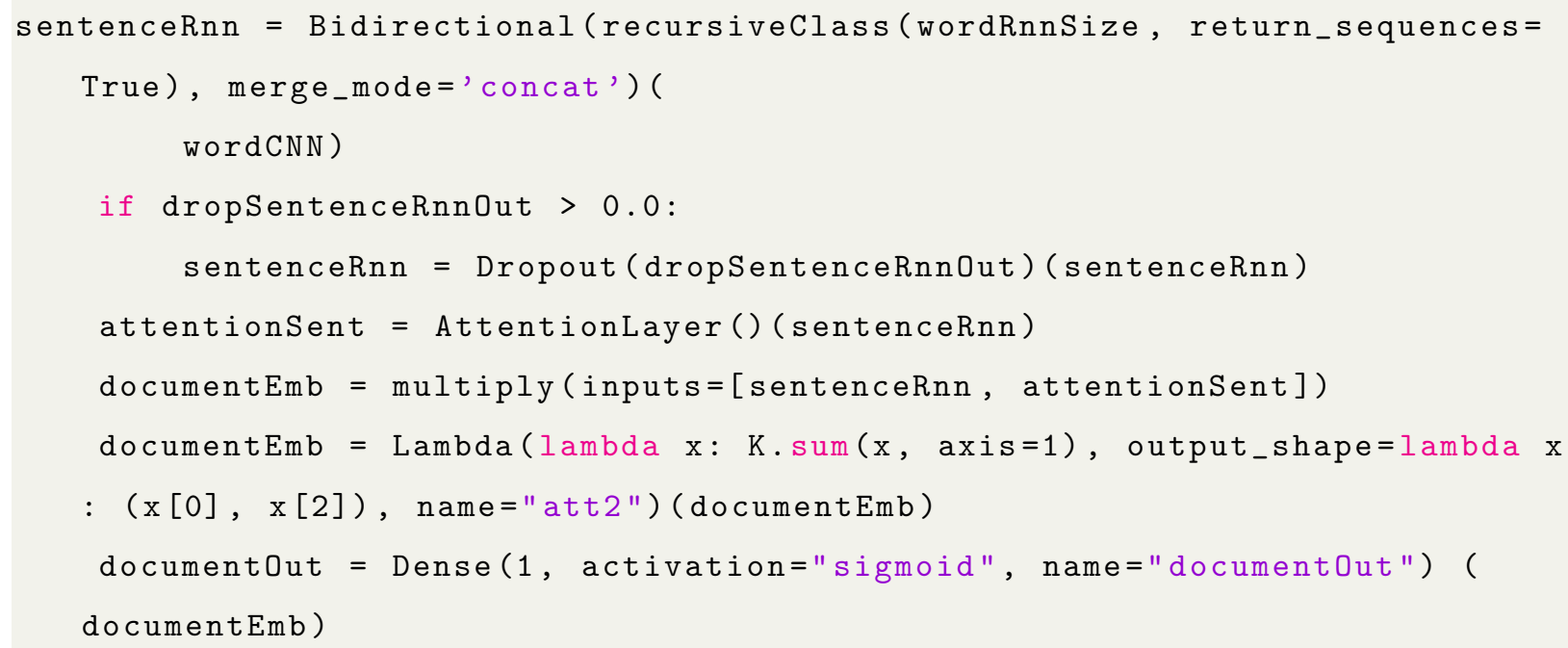

Listing 3.17: Definition parameters of bi-GRU

Listing 3.17 shows parameters of bi-GRU, which mainly helps the attention layer to extract feature in word weighted.

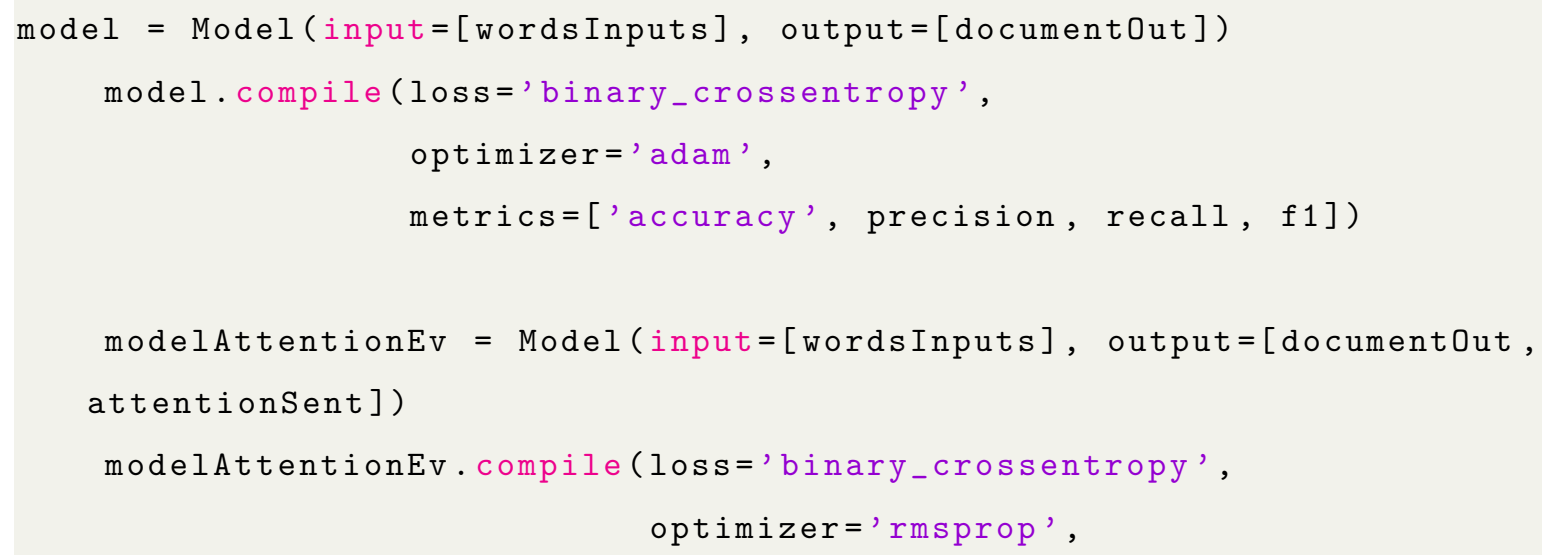




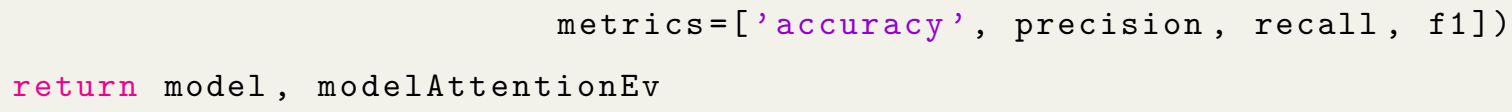

Listing 3.18: Performance evaluation.

Listing 3.18 shows performance evaluation, which evaluates each model in different data set.

\section{Experiment setting}

Table 3.17: The differences of models are used traditional machine learning and deep learning.

\begin{tabular}{|c|c|c|c|}
\hline Method & Algorithms & Parameters and their value & $\begin{array}{l}\text { Computation } \\
\text { time }\end{array}$ \\
\hline \multirow{6}{*}{$\begin{array}{l}\text { Traditional } \\
\text { machine learning }\end{array}$} & $\begin{array}{l}\text { Support vector machine } \\
\text { (SVM) }\end{array}$ & $\begin{array}{c}\mathrm{C}=23, \text { polynomial kernel function with } \\
\text { exponent }=2, \mathrm{RBF} \text { kernel function with } \\
\text { gamma }=0.01\end{array}$ & $4.5 \mathrm{~min}$ \\
\hline & Decision tree $(\mathrm{DT})$ & Period $=200$, Gain splitting $=0.01$ & $2.5 \mathrm{~min}$ \\
\hline & Logistical regression $(\mathrm{LR})$ & $\begin{array}{l}\text { The algorithm of } \\
\text { Broyden-Fletcher-Goldfarb-Shanno }\end{array}$ & $1.2 \mathrm{~min}$ \\
\hline & Random forest (RF) & $\begin{array}{l}\text { Maximum depth of trees unlimited, no. of } \\
\text { trees to be generated }=100\end{array}$ & $1.5 \mathrm{~min}$ \\
\hline & AdaBoost (AB) & no. of iterations $=10$ & $5 \mathrm{~min}$ \\
\hline & Gradient Boosting (GB) & $\begin{array}{l}\text { no. of iterations }=10 \text { and no. of } \\
\text { subcommittees }=3\end{array}$ & $3.5 \mathrm{~min}$ \\
\hline \multirow[t]{4}{*}{ Deep learning } & $\begin{array}{l}\text { Convolutional neural } \\
\text { network (CNN) }\end{array}$ & $\begin{array}{c}\text { Batch size }=16, \text { embedding size }=100 \\
\text { epoch }=20, \text { hidden size }=100, \text { output size } \\
=2, \text { optimizer: Adam, loss Function: } \\
\text { Entropy Loss, kernel width }=3,4,5 \\
\text { kernel number }=3, \text { max sentence length } \\
=200\end{array}$ & $55 \mathrm{~min}$ \\
\hline & $\begin{array}{l}\text { Long short-term memory } \\
\text { (LSTM) }\end{array}$ & $\begin{array}{l}\text { Batch size }=16, \text { embedding size }=100, \\
\text { epoch }=20, \text { hidden size }=100, \text { output } \\
\text { size }=2 \text {, optimizer: Adam, Loss Function: } \\
\text { Entropy Loss, max sentence length }=200\end{array}$ & $65 \mathrm{~min}$ \\
\hline & $\begin{array}{l}\text { Hierarchical two-layer } \\
\text { attention network } \\
\text { (HTAN) }\end{array}$ & In Section 3.2.3 & $136 \mathrm{~min}$ \\
\hline & $\begin{array}{l}\text { Hierarchical convoluted } \\
\text { attention network } \\
\text { (HCAN) }\end{array}$ & In Section 3.2 .3 & $152 \mathrm{~min}$ \\
\hline
\end{tabular}

This section compares the performance between traditional machine learning and deep learning. In Table 3.17, there are six algorithms as a baseline in traditional machine learning, such as SVM, DT, LR, RF, AB and GB. Meanwhile, four algorithms relevant deep learning in 
two cases, which are CNN, LSTM, HTAN and HCAN. The experiment results demonstrate that deep learning with attention mechanism is superior to traditional machine learning by a bag-of-words approach. Furthermore, the result of HCAN model fully illustrates the advantages of attention mechanisms and further improves the performance of deep learning. It constantly regulates the parameters and chooses the optimal parameter values.

The baseline as traditional machine learning adopts Python with Scikit-learn in this experiment. Meanwhile, deep learning utilizes Python with TensorFlow 1.6 and Keras. There is a large size of review corpus in these cases, moreover, and the deep learning algorithm requires intensive computing. Wherefore, the data preprocessing and model training was implemented with a computing system involve of a 28-core Intel Xeon E5-2683 v3 CPU with 2.00GHz and 64GB RAM. This running system is Ubuntu Linux 16.04 64-bit version. Finally, it employs the three metrics for the studio's performance, there are accuracy, sensitivity and specificity in two cases study. 


\section{Chapter

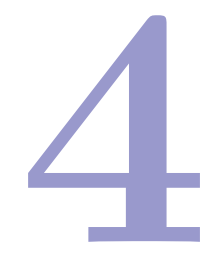

\section{Results of the research}

Table 4.1: Case study in the three application scenarios.

\begin{tabular}{cccc}
\hline & Application scenario 1 & Application scenario 2 & Application scenario 3 \\
\hline Case study & Cable News Network, & Box office, stock & audience sentiment, \\
& Thomson Reuters & market & stock market \\
\hline
\end{tabular}

This chapter describes the different cases in three application scenarios, and it also discusses the experimental results in the case study in Table 4.1.

\subsection{Application scenario 1}

This application scenario is divided into two case studies, which is mainly different data sources. One is Thomson Reuters case study, other one is Cable News Network.

\subsubsection{Thomson Reuters case study}

Three renowned companies on which the website reports were selected for the analysis. The first is Apple Inc., which is an American multinational technology company that designs, develops and sells consumer electronics, computer software and online services. The second company selected is Microsoft, which is an American multinational technology company that 
4.1. Application scenario 1

Table 4.2: Results for data extracted from Thomson Reuters.

\begin{tabular}{|c|c|c|c|c|c|}
\hline \multicolumn{2}{|c|}{ Algorithm Company } & \multirow{2}{*}{$\begin{array}{l}\text { Stock data(SD) } \\
\text { Accuracy/ } \\
\text { F1-Score }\end{array}$} & \multirow{2}{*}{$\begin{array}{c}\text { Bag of words } \\
\text { (BOW) } \\
\begin{array}{c}\text { Accuracy/ } \\
\text { F1-Score }\end{array}\end{array}$} & \multirow{2}{*}{$\begin{array}{c}\text { Convolutional } \\
\text { neural network } \\
(\mathrm{CNN})\end{array}$} & \multirow{2}{*}{$\begin{array}{c}\text { Feature } \\
\text { combine model } \\
(\mathrm{FC}) \\
\begin{array}{c}\text { Accuracy/ } \\
\text { F1-Score }\end{array}\end{array}$} \\
\hline & & & & & \\
\hline \multirow{3}{*}{ DT } & Apple & $0.4993 / 0.5079$ & $0.4889 / 0.2460$ & $0.6150 / 0.5814$ & $0.6524 / 0.6486$ \\
\hline & Microsoft & $0.4868 / 0.1819$ & $0.5065 / 0.5682$ & $0.5817 / 0.5915$ & $0.6587 / 0.6502$ \\
\hline & Samsung & $0.4707 / 0.4033$ & $0.5654 / 0.5830$ & $0.5529 / 0.6820$ & $0.5680 / 0.6260$ \\
\hline \multirow{3}{*}{ LR } & Apple & $0.5240 / 0.6727$ & $0.5055 / 0.5083$ & $0.6845 / 0.6911$ & $0.7326 / 0.7368$ \\
\hline & Microsoft & $0.4757 / 0.1253$ & $0.4430 / 0.4884$ & $0.5274 / 0.5333$ & $0.5467 / 0.5611$ \\
\hline & Samsung & $0.4947 / 0.3755$ & $0.6054 / 0.6726$ & $0.6541 / 0.6343$ & $0.8053 / 0.7978$ \\
\hline \multirow{3}{*}{ NB } & Apple & $0.5109 / 0.5123$ & $0.4778 / 0.3896$ & $0.5027 / 0.4497$ & $0.5122 / 0.4030$ \\
\hline & Microsoft & $0.4840 / 0.0363$ & $0.4557 / 0.4055$ & $0.4599 / 0.2889$ & $0.5000 / 0.5439$ \\
\hline & Samsung & $0.5293 / 0.5810$ & $0.5144 / 0.6130$ & $0.5048 / 0.6578$ & $0.5117 / 0.6601$ \\
\hline \multirow{3}{*}{$\mathrm{RF}$} & Apple & $0.5240 / 0.6554$ & $0.5278 / 0.6083$ & $0.5337 / 0.6232$ & $0.6203 / 0.6243$ \\
\hline & Microsoft & $0.4840 / 0.2653$ & $0.4177 / 0.1687$ & $0.5325 / 0.2432$ & $0.5721 / 0.5189$ \\
\hline & Samsung & $0.4872 / 0.3480$ & $0.4485 / 0.1194$ & $0.4766 / 0.3708$ & $0.5052 / 0.5481$ \\
\hline \multirow{3}{*}{ GD } & Apple & $0.4672 / 0.1119$ & $0.4778 / 0.4404$ & $0.4974 / 0.6008$ & $0.5366 / 0.2692$ \\
\hline & Microsoft & $0.5173 / 0.6819$ & $0.4852 / 0.3222$ & $0.6103 / 0.4231$ & $0.6108 / 0.4193$ \\
\hline & Samsung & $0.5223 / 0.6541$ & $0.4766 / 0.2000$ & $0.5187 / 0.6751$ & $0.5571 / 0.6548$ \\
\hline \multirow{3}{*}{ SVM } & Apple & $0.5022 / 0.6121$ & $0.5747 / 0.5542$ & $0.6150 / 0.6129$ & $0.6220 / 0.6173$ \\
\hline & Microsoft & $0.4784 / 0.1722$ & $0.4439 / 0.4566$ & $0.5232 / 0.5462$ & $0.6875 / 0.6798$ \\
\hline & Samsung & $0.5008 / 0.3875$ & $0.5047 / 0.5508$ & $0.5135 / 0.6281$ & $0.5352 / 0.6574$ \\
\hline \multirow{3}{*}{$\mathrm{AB}$} & Apple & $0.5343 / 0.5384$ & $0.4722 / 0.2963$ & $0.4819 / 0.3333$ & $0.6203 / 0.5420$ \\
\hline & Microsoft & $0.4687 / 0.2041$ & $0.4768 / 0.0461$ & $0.6147 / 0.6044$ & $0.6202 / 0.6146$ \\
\hline & Samsung & $0.4857 / 0.3619$ & $0.4579 / 0.2368$ & $0.6010 / 0.6820$ & $0.6338 / 0.6549$ \\
\hline \multirow{3}{*}{ GB } & Apple & $0.5314 / 0.5129$ & $0.4667 / 0,2941$ & $0.6096 / 0.4748$ & $0.6341 / 0.6591$ \\
\hline & Microsoft & $0.4743 / 0.2125$ & $0.4728 / 0.1007$ & $0.4557 / 0.4557$ & $0.5385 / 0.2258$ \\
\hline & Samsung & $0.4872 / 0.4171$ & $0.5192 / 0.6599$ & $0.5337 / 0.6572$ & $0.5622 / 0.6335$ \\
\hline
\end{tabular}

develops, manufactures, supports and sells computer software, personal computers and other services. The last company chosen is Samsung, which is a South Korean multinational electronics company. This study considered data from these three typical technology companies for the problem.

Measurements of average accuracy are shown in Table 4.2, and the results for each renowned company with respect to prediction based on different features are illustrated in Figure 4.1. It calculated the average of the four feature construction approaches for each model for comparison amongst the three companies. The proposed feature combination 


\section{Reuters}

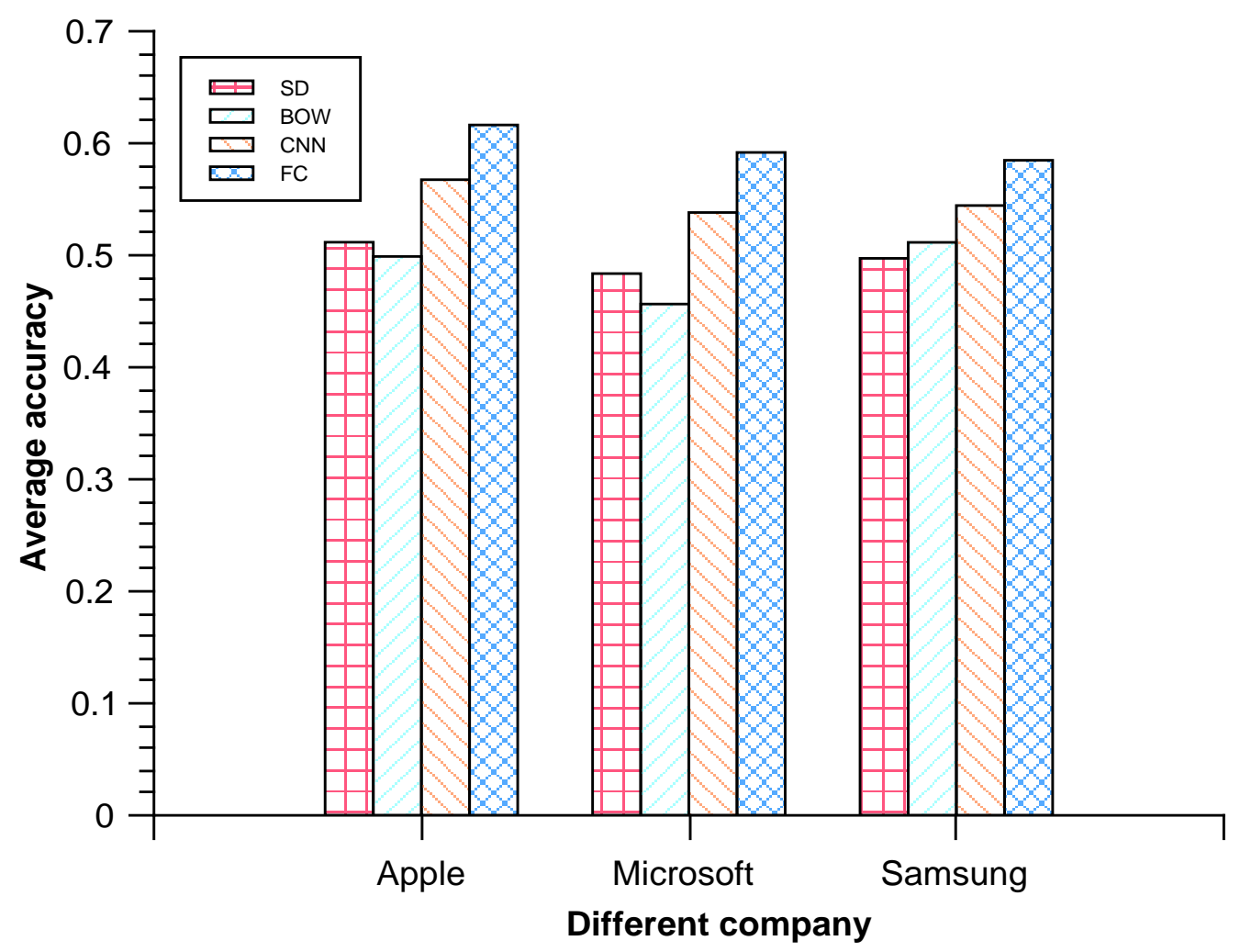

Figure 4.1: Average accuracy of prediction based on different feature sets for each company in Reuters. 
model generated the best results, achieving average accuracy levels of $61.63 \%, 59.18 \%$ and $58.48 \%$ for Apple, Microsoft and Samsung, respectively. These figures are consistent with the rates reported in previous research. However, many studies analyzed only one company or used only one algorithm. The current research probed into three companies on the basis of information from a common data source to build a prediction model with different feature selection functionalities and different algorithms. It used stock data, a bag-of-words algorithm, a convolutional neural network and feature combination, together with eight algorithms. As indicated in Table 4.2, the proposed prediction model achieved $73.68 \%$ data extraction with the use of LR_4 for Apple and 67.78\% data extraction with the use of SVM_4 for Microsoft. In particular, the LR algorithm used for Apple achieved accuracy and F1-score of 0.7326 and 0.7360, respectively, which highlights its powerful capability in a two-class classification.

To assess the effectiveness of this research, It compared the feature combination model that uses event tuples with the stock data approach and the bag-of-words algorithm. The results showed that the average accuracy of the event tuple-based model is better than that of the stock data approach and the bag-of-words algorithm by $10.01 \%$ and $10.87 \%$, respectively. It also compared feature extraction using the stock data approach, the bagof-words algorithm and machine learning. The use of deep learning improved prediction accuracy by $5.25 \%$ and $6.11 \%$ over the levels achieved with stock data and bag of words, respectively. Therefore, this study can conclude that using the proposed feature combination model and deep learning in feature extraction helps improve the accuracy with which stock price movement is predicted. These results also fully prove the effectiveness of embedding layer in feature extraction accuracy improvement.

\subsubsection{Cable News Network case study}

Three renowned companies on which these website reports were selected for the analysis. The first is Boeing, which is an American multinational corporation that designs, manufactures and sells airplanes, rotorcraft, rockets and satellites worldwide. The second company is Google, which is an American multinational technology company that specializes in Internet-related services and products. The third is Walmart, an American multinational retail corporation that operates a chain of hypermarkets, discount department stores and 
Chapter 4. Results of the research

Table 4.3: Results for data extracted from Cable News Network.

\begin{tabular}{|c|c|c|c|c|c|}
\hline \multicolumn{2}{|c|}{ Algorithm Company } & \multirow{2}{*}{$\begin{array}{l}\text { Stock data(SD) } \\
\text { Accuracy/ } \\
\text { F1-Score }\end{array}$} & \multirow{2}{*}{$\begin{array}{c}\text { Bag of words } \\
\text { (BOW) } \\
\begin{array}{c}\text { Accuracy/ } \\
\text { F1-Score }\end{array}\end{array}$} & \multirow{2}{*}{$\begin{array}{c}\text { Convolutional } \\
\text { neural network } \\
(\mathrm{CNN})\end{array}$} & \multirow{2}{*}{$\begin{array}{c}\text { Feature } \\
\text { combine model } \\
(\text { FC }) \\
\begin{array}{c}\text { Accuracy/ } \\
\text { F1-Score }\end{array}\end{array}$} \\
\hline & & & & & \\
\hline \multirow{3}{*}{ DT } & Boeing & $0.4950 / 0.3489$ & $0.5413 / 0.6258$ & $0.5414 / 0.6347$ & $0.5591 / 0.5821$ \\
\hline & Google & $0.4858 / 0.5600$ & $0.5182 / 0.3575$ & $0.5692 / 0.4631$ & $0.5491 / 0.5471$ \\
\hline & Walmart & $0.4996 / 0.4422$ & $0.5300 / 0.4891$ & $0.5088 / 0.4815$ & $0.5722 / 0.5829$ \\
\hline \multirow{3}{*}{ LR } & Boeing & $0.4923 / 0.2378$ & $0.5113 / 0.4348$ & $0.6923 / 0.6923$ & $0.7596 / 0.5733$ \\
\hline & Google & $0.5041 / 0.6105$ & $0.6235 / 0.6491$ & $0.6250 / 0.6111$ & $0.6563 / 0.6516$ \\
\hline & Walmart & $0.5290 / 0.5468$ & $0.5412 / 0.5083$ & $0.7320 / 0.7347$ & $0.7719 / 0.7720$ \\
\hline \multirow{3}{*}{ NB } & Boeing & $0.5162 / 0.6503$ & $0.5489 / 0.5161$ & $0.5197 / 0.6738$ & $0.5564 / 0.7619$ \\
\hline & Google & $0.5112 / 0.6527$ & $0.4899 / 0.5191$ & $0.5587 / 0.5281$ & $0.5344 / 0.4843$ \\
\hline & Walmart & $0.5044 / 0.3284$ & $0.4794 / 0.5302$ & $0.5206 / 0.5674$ & $0.5300 / 0.6083$ \\
\hline \multirow{3}{*}{$\mathrm{RF}$} & Boeing & $0.4906 / 0.1345$ & $0.5113 / 0.3925$ & $0.5096 / 0.5405$ & $0.5276 / 0.5714$ \\
\hline & Google & $0.4923 / 0.1777$ & $0.6032 / 0.6260$ & $0.5223 / 0.5564$ & $0.5951 / 0.5763$ \\
\hline & Walmart & $0.5118 / 0.5373$ & $0.5300 / 0.3188$ & $0.5300 / 0.4405$ & $0.5000 / 0.5446$ \\
\hline \multirow{3}{*}{ GD } & Boeing & $0.4897 / 0.2407$ & $0.5564 / 0.4870$ & $0.5433 / 0.4727$ & $0.5714 / 0.7220$ \\
\hline & Google & $0.5165 / 0.6769$ & $0.5425 / 0.4375$ & $0.5789 / 0.6364$ & $0.5910 / 0.6327$ \\
\hline & Walmart & $0.5004 / 0.3804$ & $0.5206 / 0.5280$ & $0.5250 / 0.5581$ & $0.5670 / 0.5922$ \\
\hline \multirow{3}{*}{ SVM } & Boeing & $0.4897 / 0.3068$ & $0.5714 / 0.6275$ & $0.5385 / 0.6418$ & $0.5940 / 0.7033$ \\
\hline & Google & $0.5077 / 0.6297$ & $0.6032 / 0.6230$ & $0.5749 / 0.5532$ & $0.5789 / 0.5840$ \\
\hline & Walmart & $0.5118 / 0.4333$ & $0.5000 / 0.5314$ & $0.5200 / 0.5000$ & $0.5614 / 0.5455$ \\
\hline \multirow{3}{*}{$\mathrm{AB}$} & Boeing & $0.4945 / 0.3404$ & $0.5197 / 0.5120$ & $0.4961 / 0.5294$ & $0.5673 / 0.5872$ \\
\hline & Google & $0.4876 / 0.3141$ & $0.5101 / 0.6667$ & $0.5893 / 0.5619$ & $0.5951 / 0.0685$ \\
\hline & Walmart & $0.5073 / 0.5029$ & $0.5150 / 0.5359$ & $0.5497 / 0.5650$ & $0.5619 / 0.5503$ \\
\hline \multirow{3}{*}{ GB } & Boeing & $0.4899 / 0.3169$ & $0.5118 / 0.4364$ & $0.4409 / 0.4580$ & $0.5000 / 0.5094$ \\
\hline & Google & $0.4912 / 0.3387$ & $0.5870 / 0.5854$ & $0.5625 / 0.5243$ & $0.6032 / 0.6202$ \\
\hline & Walmart & $0.5003 / 0.4955$ & $0.5050 / 0.5123$ & $0.5361 / 0.5109$ & $0.5497 / 0.5650$ \\
\hline
\end{tabular}

grocery stores.

The results on average accuracy are shown in Table 4.3 and Fig 4.2. These findings are very similar to those derived in the previous case study, and their comparison confirmed that the proposed feature combination model can outperform other feature selection models in stock price movement prediction. With regard to feature selection underlain by the bag-ofwords algorithm, the CNN case study generated more robust results than did the Reuters case study. The average accuracy of the bag-of-words algorithm was lower than that of the stock data approach in the Reuters case study. In the CNN case study, the average accuracy levels 


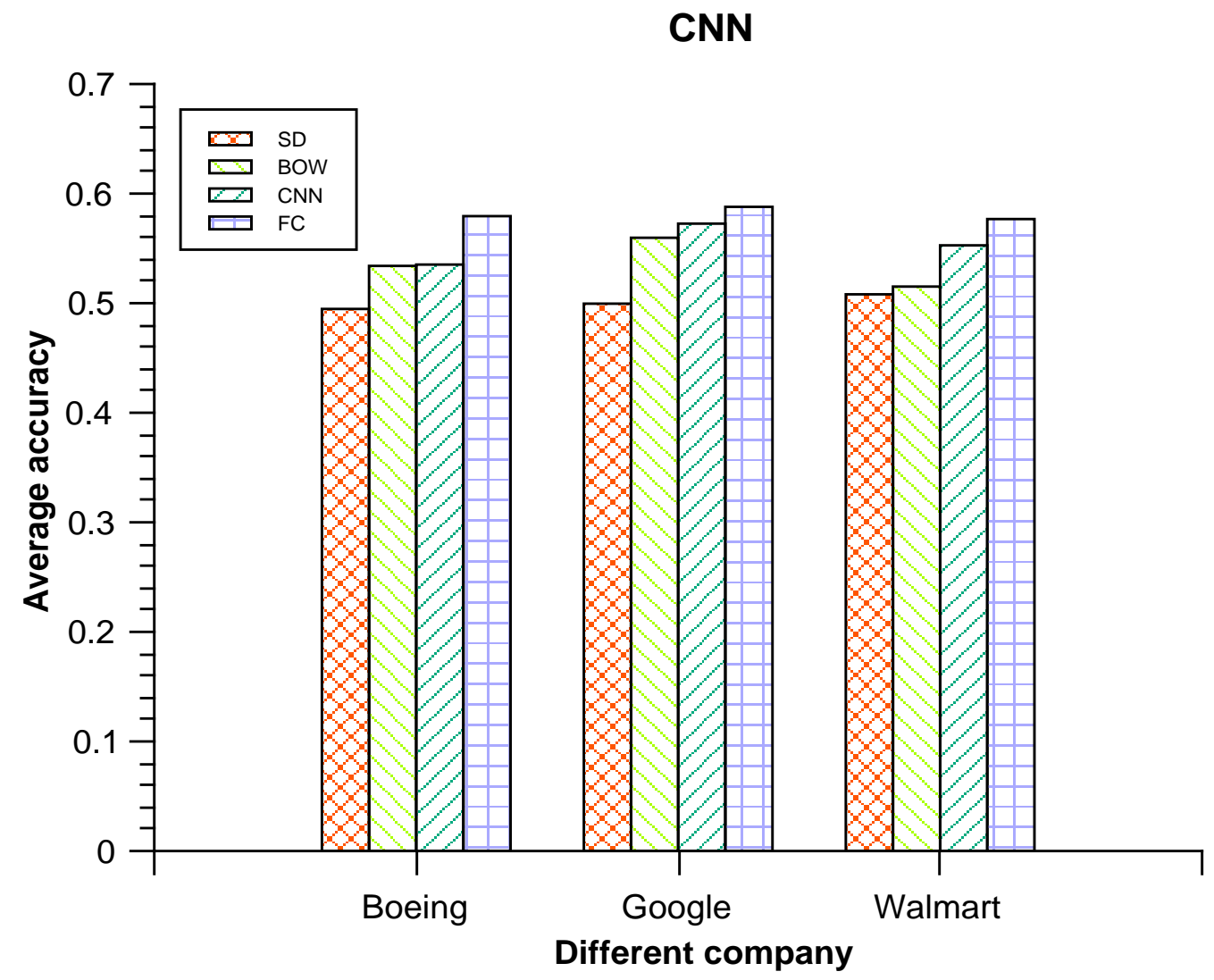

Figure 4.2: Average accuracy achieved on the basis of news from CNN and different feature set. 
of the proposed feature combination model were $57.94 \%, 58.79 \%$ and $57.67 \%$ for Boeing, Google and Walmart, respectively. In the case study on Reuters, an average accuracy that exceeds $60 \%$ was not achieved for any of the companies, illustrating that differences in data source directly affect stock price movement.

This study chooses a combination of deep learning and knowledge graph to build the feature selection model because this combination exhibited superior prediction performance in the comparison experiments involving other feature selection strategies. The forecast results based on different features are shown in Table 4.3. It found that deep learning features are better than the use of bag-of-words and stock data. The correlation of event tuples with the stock market is relatively high in 100,101], but the bag-of-words algorithm was relatively fragmented, and the correlation between event tuples and the stock market data was relatively weak. Combining event tuple features with deep learning significantly improves forecast results, indicating a close relationship between stock market movements and knowledge graph.

In this experiment of two cases, the optimal parameters are $\lambda=0.001, \mathrm{k}=100, \mathrm{~B}=$ $1440, \alpha=0.8$ (The experiment setting is in Section 3.3.2). The results of text representation and structure representation $(\mathrm{CNN}+$ TransE) are better than that of text representation in CNN training alone, illustrates that combined training can better represent the data in knowledge graph, which shows that using convolutional neural network processing for feature selection can better represent text information.

Compared machine learning for classification tasks in two cases, it finds that the results of ensemble learning are basically higher than traditional machine learning, such as AB $(59.51 \%$ of feature combine model in Router cases) and GB (60.32\% of feature combine model in Router cases). The advantages of ensemble learning are low generalization error rate and high accuracy, especially in binary classification, it will not overfitting. Different from SVM, the forecast preparation rate of GB can also be high at little adjustment time. 


\subsection{Application scenario 2}

This application scenario is divided into two case studies, which is mainly different in company performance. First case is box office, other case is stock market of the studios.

\subsubsection{Company performance in the box office}

\section{The statistics method}

This section adopted the cross-sectional regression to explore the relationship between UGC and box office revenue. The regression equations are as follows:

$$
\begin{array}{r}
\ln (\text { Rev })_{i}=\beta^{\prime} x_{i}+\gamma \ln (\text { pre_like })^{i}+\varepsilon_{i} \\
\ln (\text { Rev })_{i}=\beta^{\prime} x_{i}+\gamma \ln (\text { like })^{i}+\varepsilon_{i} \\
\ln (\text { Rev })_{i}=\beta^{\prime} x_{i}+\gamma \ln (\text { pre_share })^{i}+\varepsilon_{i} \\
\ln (\text { Rev })_{i}=\beta^{\prime} x_{i}+\gamma \ln (\text { share })^{i}+\varepsilon_{i}
\end{array}
$$

It defined the dependent variable as Rev1, Rev7 and Rev30, which denotes box office on the opening day, the opening week and the opening month. Moreover, it also defined the independent variable $\mathrm{x}$ as the number of likes and shares on Facebook. The different periods suggest that there were pre_like1, pre_like7, pre_like30, pre_share1, pre_share7 and pre_share30 in the movie before the release; conversely, there were like1, like7, like30, share1, share7 and share30 in the after-movie release. To avoid endogeneity in the regression model, it also created some control variables from the movie performance, such as budget, theater, Friday (movies are usually released on Friday in the United States, hence label one (1) and zero (0) otherwise), MPAA ratings and genre. 


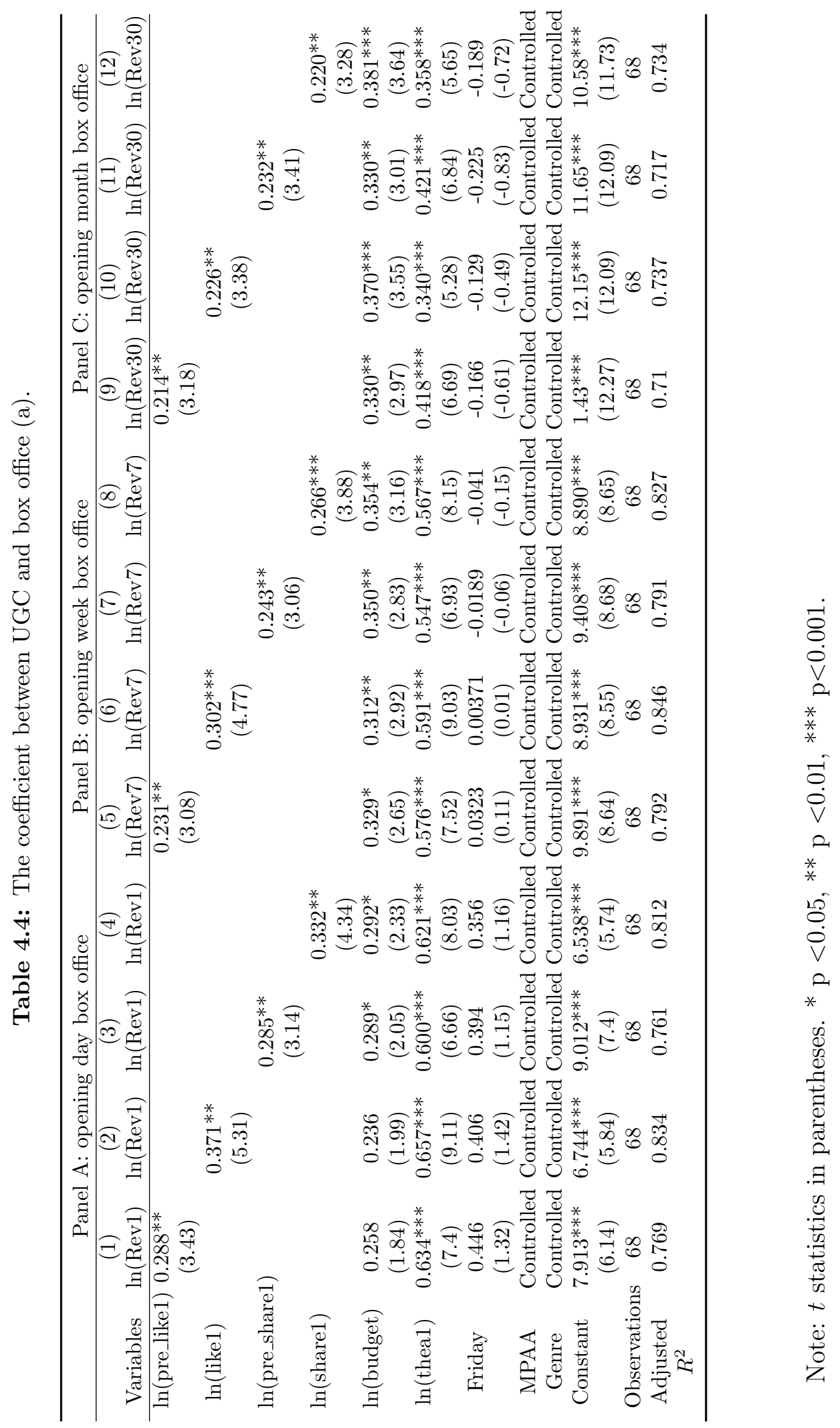




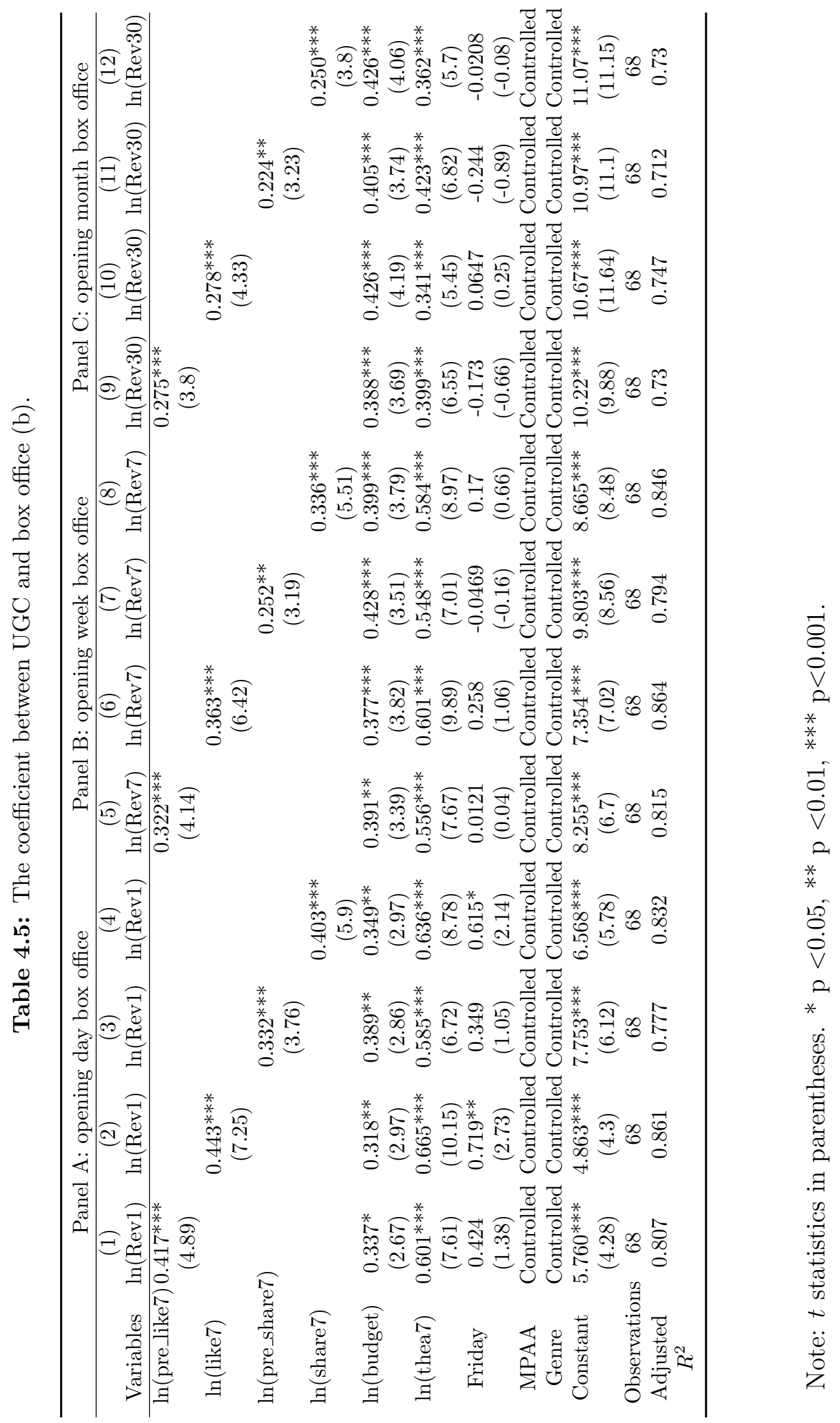




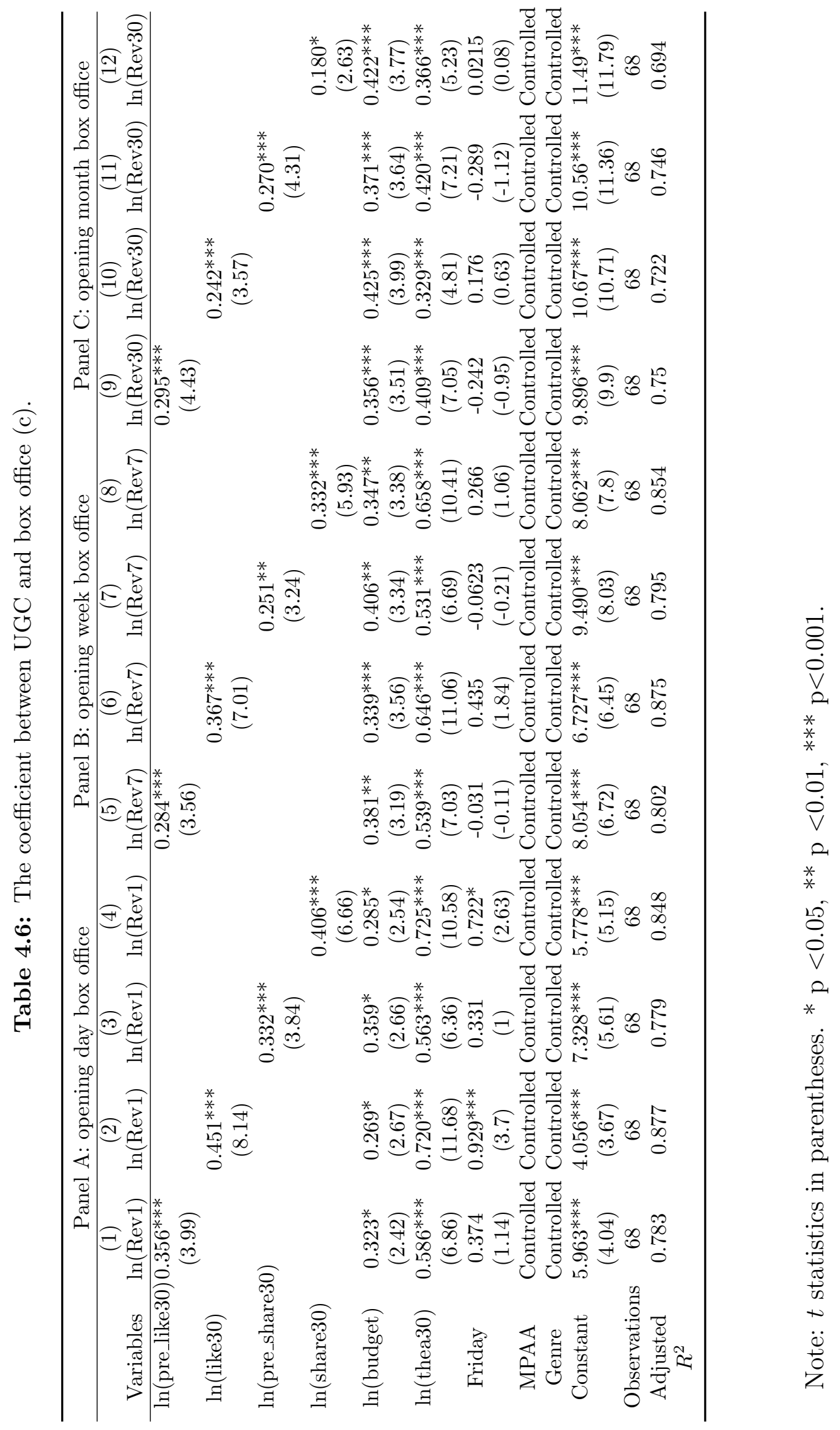


Based on the proposed equation model, Tables 4.4, 4.5 and 4.6 present the results of the empirical analysis through OLS regression. It found that likes and shares had a positive influence on the box office. Model (1) in Table 4.4 shows the coefficient of 0.288 and 0.371 for pre_like1 and like1, which are positive at 1\% level. In panel B, there are 0.302 and 0.266 for like1 and share1, respectively. Since shares and likes have a high degree of relevance, with very similar coefficients, it inferred that both had a positive effect on the box office. Additionally, theater, a variable, is the highest positive that yielded $0.1 \%$ and had a substantial impact on the box office in each model. The other control variable, budget, also had a positive effect on the box office; however, Friday, as another variable, did not show an explicit connection. The results in Tables 4.4 and 4.5 are in line with the expectation; the coefficient of the three tables is the same. On the other hand, across the three tables, the variable correlation in Tables 4.4 and 4.5 are significantly higher than Table 4.6 because most of the coefficients were achieved at $0.1 \%$ level.

This section introduced the number of likes and shares both before and after the release of a movie. Moreover, from the psychological point of view, likes are more likely than shares in the degree of general expression. These empirical results also confirm that the coefficients of likes are more than the coefficients of shares in most models; this result not only enriches the data diversity but also reflects the characteristics of the audience's sentiment. In conclusion, the empirical model reflects the real data of the box office, and this finding illustrates a positive relationship between audience engagement and studio performance.

\section{The machine learning method}

The accuracy evaluation results are shown in Table 4.7. Additionally, it also computed the average accuracy of each studio. Most of the average accuracy was around $75 \%$. In the case of the audience's rating-based method, the average accuracy of Fox (77.61\%) was significantly higher than the other studios. The sentiment words were implicit and objective, which was directly useful to judge the audience's review by sentiment dictionary. Given the subjectivity of the human's emotional expression, which influences the consistency between the audience's review and the audience's rating, the flexibility of the rating-based method has 
Chapter 4. Results of the research

Table 4.7: The accuracy and F1-Score of classification of the audience's review.

\begin{tabular}{|c|c|c|c|c|c|c|c|}
\hline \multirow{2}{*}{$\begin{array}{c}\text { Studios } \\
\text { Studio }\end{array}$} & \multirow{2}{*}{$\begin{array}{c}\text { Baseline } \\
(\%) \\
\text { MLR }\end{array}$} & \multicolumn{6}{|c|}{ Classical machine learning (\%) } \\
\hline & & LR & $\mathrm{RF}$ & XGB & GNB & SVM & Average \\
\hline & \multicolumn{7}{|c|}{ Accuracy/F1Accuracy/F1Accuracy/F1Accuracy/F1Accuracy/F1Accuracy/F1Accuracy/F1 } \\
\hline & Score & Score & Score & Score & Score & Score & Score \\
\hline Disney & $52.91 / 54.03$ & $83.65 / 83.70$ & $75.61 / 76.67$ & $72.59 / 80.13$ & $68.19 / 66.87$ & $83.21 / 83.80$ & $72.69 / 74.20$ \\
\hline Fox & $59.31 / 58.94$ & $85.94 / 82.67$ & $79.12 / 77.16$ & $74.99 / 84.03$ & $73.19 / 70.15$ & $86.19 / 82.92$ & $76.46 / 75.98$ \\
\hline Lionsgate & $83.97 / 83.70$ & $83.65 / 80.06$ & $77.85 / 76.01$ & $73.53 / 83.31$ & $73.48 / 69.97$ & $81.19 / 80.55$ & $78.95 / 78.94$ \\
\hline Paramount & $50.04 / 53.03$ & $81.72 / 80.24$ & $73.09 / 73.50$ & $71.27 / 80.37$ & $69.79 / 70.38$ & $80.63 / 79.77$ & $71.09 / 72.89$ \\
\hline Columbia & $58.48 / 61.12$ & $79.67 / 81.83$ & $74.72 / 73.99$ & $70.97 / 81.10$ & $70.48 / 74.05$ & $80.05 / 83.11$ & $72.40 / 75.87$ \\
\hline Universal & $56.97 / 60.52$ & $83.60 / 85.51$ & $78.67 / 80.96$ & $78.52 / 86.84$ & $70.83 / 73.32$ & $84.82 / 85.65$ & $75.57 / 78.80$ \\
\hline Warner & $57.21 / 57.34$ & $83.94 / 82.84$ & $79.34 / 77.10$ & $77.34 / 85.94$ & $71.57 / 69.65$ & $84.11 / 83.52$ & $75.59 / 76.07$ \\
\hline
\end{tabular}

more advantages for two-class sentiment, which provides a granular emotional understanding. Therefore, the elaborate analysis results can help studios understand the emotional fluctuations of the audience and the box office trend.

To assess the effectiveness of classical machine learning in audience sentiment of UGC, it compared the average accuracy and average F1-Score based on different algorithms in Figures 4.3. The results indicated that the average F1-Score was very similar to accuracy. This study found that the overall performance of the classical machine learning model exceeded the baseline, which provides strong evidence that machine learning has the apparent advantage of two-classification. Using LR, the average of the results are higher than the other machine learning methods. Not all linear models can guarantee that the range of predicted values will lie between 0 and 1 . LR is a logarithmic linear model that is suitable for a task of probabilistic classification. Their output has a reasonable probability of interpretation, and the algorithm can also be regularized to avoid over-fitting. The logistical model easily updates the data model by random gradient descent. Besides, these models are robust for small noise in the review corpus and are not particularly impacted by slight multicollinearity.

Most of the audience's sentiment in review corpus is implicit 198, which makes it difficult to directly judge its characteristics and further impacts the feature extraction in UGC. Based on the classification capability of machine learning for the audience's sentiment, this study found the detailed characteristics of the audience's sentiment through audience review corpus, 


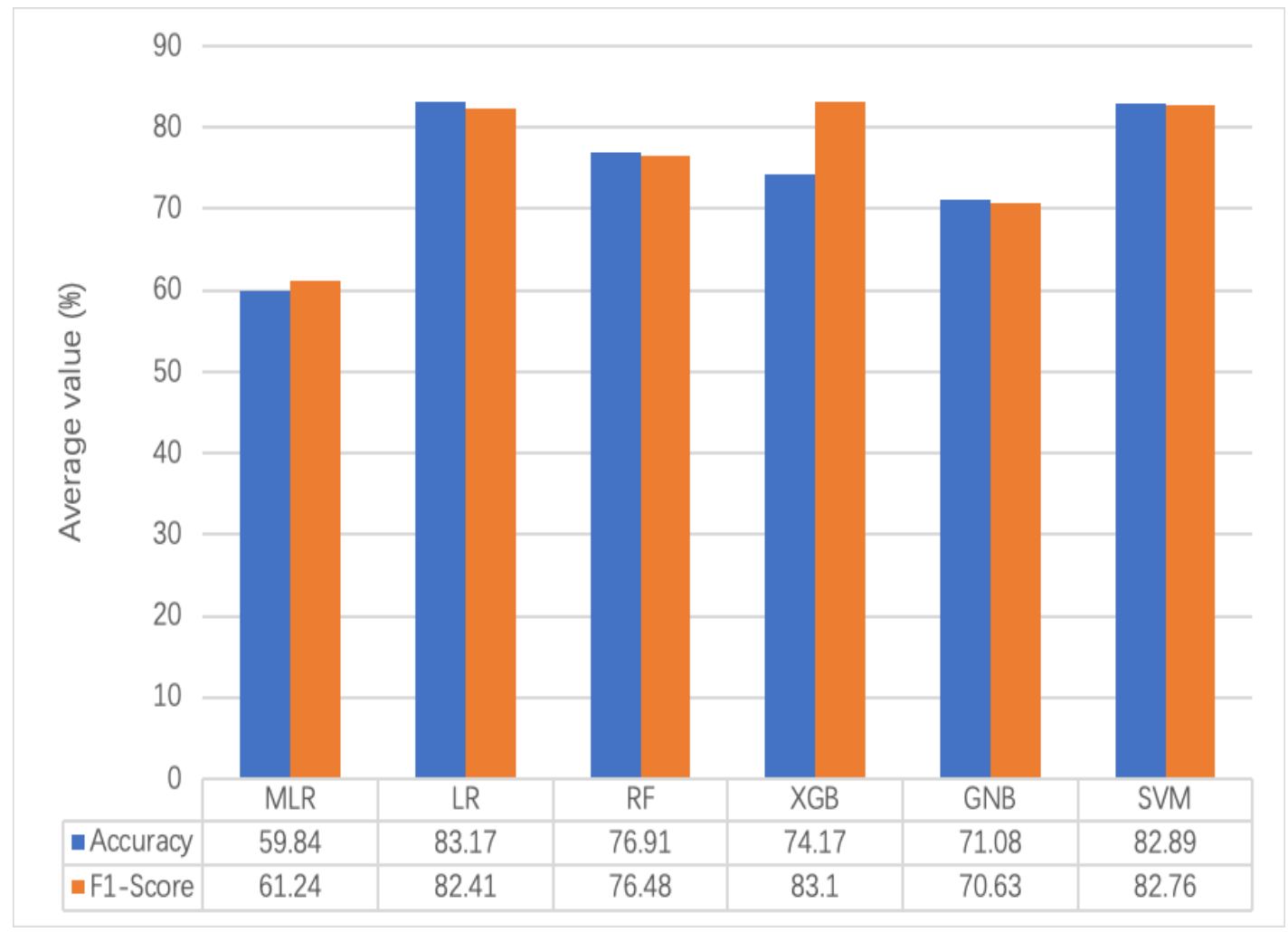

Figure 4.3: Average accuracy and F1-Score for each algorithm in the classification of audience's review through the rating-based method. 
which can help studios understand the emotional trend of the audience in real-time. Overall, using classical machine learning helps improve performance in the classification task of the audience's sentiment, which furnishes decision support for UGC in social media.

\subsubsection{Company performance in the stock market}

\section{The statistics method}

In this section, which reveals the relationship between UGC and the stock market using OLS regression. The following equation estimates the coefficient thus:

$$
\begin{array}{r}
\ln (\text { Return })_{i}=\beta^{\prime} x_{i}+\gamma \ln (\text { pre_like })^{i}+\varepsilon_{i} \\
\ln (\text { Return })_{i}=\beta^{\prime} x_{i}+\gamma \ln (\text { pre_share })^{i}+\varepsilon_{i} \\
\text { Return7 }=\log \frac{\text { close price }_{t+7}}{\text { close price }_{t}} \\
\text { Return30 }=\log \frac{\text { close price }_{t+30}}{\text { close price }_{t}}
\end{array}
$$

The dependent variable 'Return' is the stock return on the opening day (Return1), the opening week (Return7) and the opening month (Return30). It only created different independent variables such as pre_like and pre_share in a different period. Behavioral finance holds that positive information is strengthened in the period of rising investor sentiment. The investors are optimistic about the listed companies' earnings prospects; other investors are constantly affected by their sentiment. Moreover, new investors have actively entered the market, which continues to drive up the stock price movement, hence the time lag relationship between the emotion and the stock price.

Tables 4.8, 4.9 and 4.10 show that these results utilize explanatory variables such as pre_like and pre_share for exploring the above problems. To avoid the endogeneity caused by missing variables, it introduced the variable of the market factor, Re_sp, to influence the studio's stock price and other control variables (e.g. studio). Moreover, it emphasizes two 
Table 4.8: The coefficient between UGC and stock market (a).

\begin{tabular}{|c|c|c|c|c|c|c|}
\hline & (1) & $(2)$ & $(3)$ & (4) & (5) & (6) \\
\hline Variables & $\ln ($ Return1) & $\ln ($ Return1) & $\ln ($ Return1) & $\ln ($ Return1) & $\ln ($ Return1) & $\overline{\ln (\text { Return1 })}$ \\
\hline $\ln ($ pre_like1) & $\begin{array}{c}0.000607 \\
(1.36)\end{array}$ & & & & & \\
\hline $\ln ($ pre_like7) & & $\begin{array}{c}0.00105^{* *} \\
(2.13)\end{array}$ & & & & \\
\hline $\ln ($ pre_like30) & & & $\begin{array}{c}0.000406 \\
(0.78)\end{array}$ & & & \\
\hline $\ln ($ pre_share1) & & & & $\begin{array}{c}0.000454 \\
(1.01)\end{array}$ & & \\
\hline ln(pre_share7) & & & & & $\begin{array}{c}0.00111^{* *} \\
-2.41\end{array}$ & \\
\hline $\ln ($ pre_share 30$)$ & & & & & & $\begin{array}{c}0.000385 \\
(0.77)\end{array}$ \\
\hline re_sp1 & $\begin{array}{l}-0.134 \\
(-0.45)\end{array}$ & $\begin{array}{l}-0.179 \\
(-0.61)\end{array}$ & $\begin{array}{c}-0.0865 \\
(-0.29)\end{array}$ & $\begin{array}{c}-0.0988 \\
(-0.33)\end{array}$ & $\begin{array}{l}-0.175 \\
(-0.61)\end{array}$ & $\begin{array}{c}-0.0931 \\
(-0.31)\end{array}$ \\
\hline Friday & $\begin{array}{c}0.000938 \\
(0.5)\end{array}$ & $\begin{array}{c}0.00105 \\
(0.57)\end{array}$ & $\begin{array}{c}0.00114 \\
(0.6)\end{array}$ & $\begin{array}{c}0.000805 \\
(0.42)\end{array}$ & $\begin{array}{c}0.000548 \\
(0.3)\end{array}$ & $\begin{array}{c}0.000958 \\
(0.5)\end{array}$ \\
\hline $\begin{array}{c}\text { Studio } \\
\text { Constant }\end{array}$ & $\begin{array}{c}\text { Controlled } \\
-0.00181 \\
(-0.36)\end{array}$ & $\begin{array}{c}\text { Controlled } \\
-0.0123^{*} \\
(-1.85)\end{array}$ & $\begin{array}{c}\text { Controlled } \\
-0.00114 \\
(-0.16)\end{array}$ & $\begin{array}{c}\text { Controlled } \\
0.000672 \\
-0.16\end{array}$ & $\begin{array}{c}\text { Controlled } \\
-0.0059 \\
(-1.19)\end{array}$ & $\begin{array}{c}\text { Controlled } \\
-0.00377 \\
(-0.59)\end{array}$ \\
\hline Observations & 68 & 68 & 68 & 68 & 68 & 68 \\
\hline $\begin{array}{c}\text { Adjusted } \\
R^{2}\end{array}$ & 0.026 & 0.068 & 0.006 & 0.013 & 0.087 & 0.005 \\
\hline
\end{tabular}

interesting conclusions. First, the cumulative pre_like number and the pre_share number in the week period are positively related to stock return from all the models. Second, the correlation of Return7 is the highest across the three tables; this suggests that the audience's sentiment has a positive impact on the stock return, which causes the stock price to increase. The emotional release time lag maybe one week.

The comparison between the stock market and box office revenue shows that the box office is more responsive to the audience's sentiment. The stock market of the studios is complicated and mutative, and the stock returns are affected by various factors, except for the audience's sentiment, the studio's operating conditions, development prospects and dividend distribution policy. 
Chapter 4. Results of the research

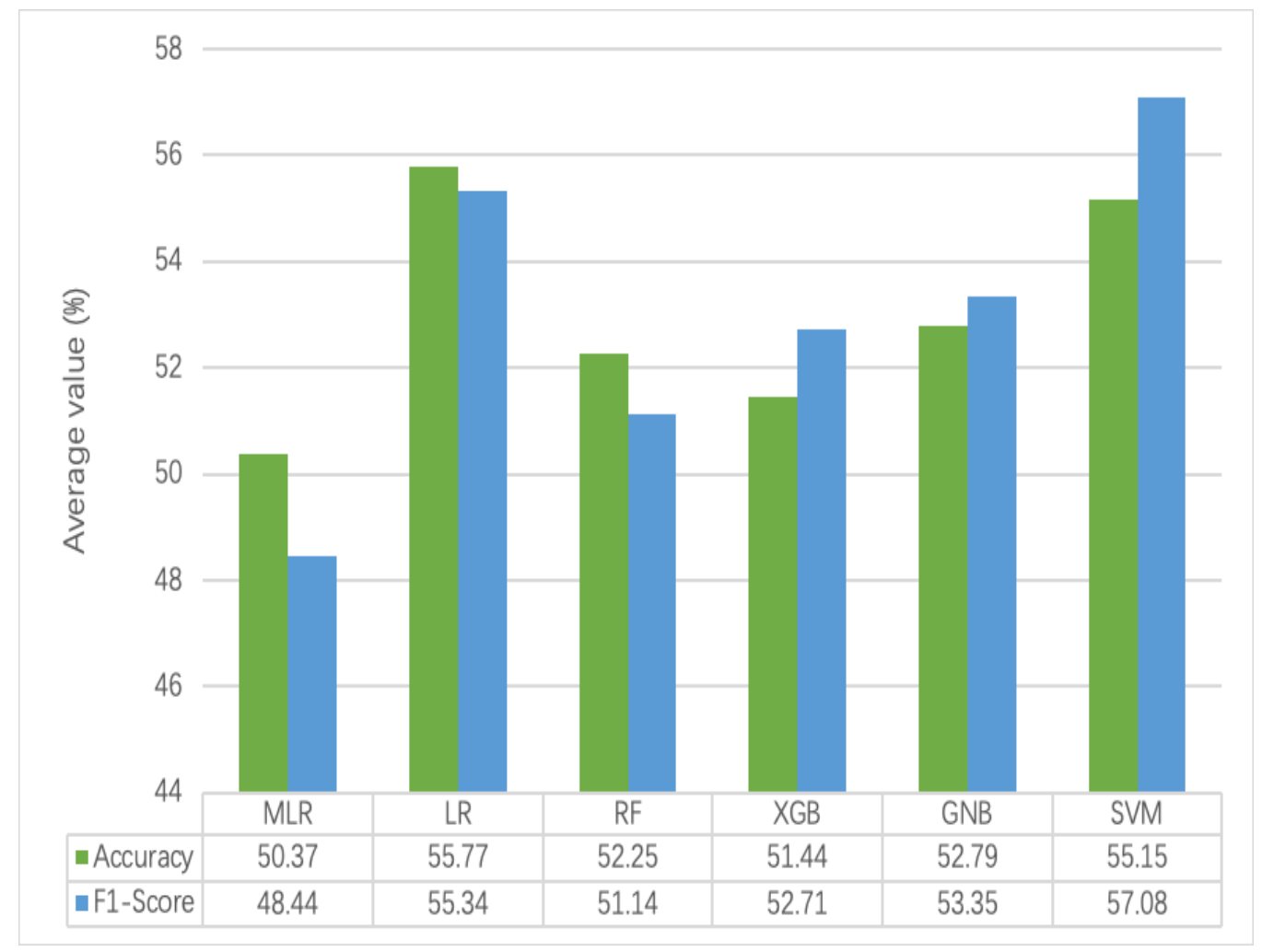

Figure 4.4: Average accuracy and F1-Score for each algorithm in the prediction of stock price movement. 
Table 4.9: The coefficient between UGC and stock market (b).

\begin{tabular}{|c|c|c|c|c|c|c|}
\hline & (1) & (2) & $(3)$ & (4) & (5) & (6) \\
\hline Variables & $\ln ($ Return7) & $\ln ($ Return7) & $\ln ($ Return7) & $\ln ($ Return7) & $\ln ($ Return7) & $\ln ($ Return7) \\
\hline $\ln ($ pre_like1) & $\begin{array}{c}0.00233^{* *} \\
(2.45)\end{array}$ & & & & & \\
\hline $\ln ($ pre_like7) & & $\begin{array}{c}0.00318^{* * *} \\
(3.03)\end{array}$ & & & & \\
\hline $\ln ($ pre_like30) & & & $\begin{array}{c}0.00191^{*} \\
(1.68)\end{array}$ & & & \\
\hline $\ln ($ pre_share 1$)$ & & & & $\begin{array}{c}0.00208^{* *} \\
(2.16)\end{array}$ & & \\
\hline $\ln ($ pre_share 7$)$ & & & & & $\begin{array}{c}0.00321^{* * *} \\
(3.28)\end{array}$ & \\
\hline $\ln$ (pre_share30 & & & & & & $\begin{array}{c}0.00185^{*} \\
(1.71)\end{array}$ \\
\hline re_sp7 & $\begin{array}{c}0.871^{* * *} \\
(3.46)\end{array}$ & $\begin{array}{c}0.851^{* * *} \\
(3.46)\end{array}$ & $\begin{array}{c}0.900^{* * *} \\
(3.47)\end{array}$ & $\begin{array}{c}0.891^{* * *} \\
(3.51)\end{array}$ & $\begin{array}{c}0.865^{* * *} \\
(3.57)\end{array}$ & $\begin{array}{c}0.901^{* * *} \\
(3.48)\end{array}$ \\
\hline Friday & $\begin{array}{c}-0.00124 \\
(-0.31)\end{array}$ & $\begin{array}{c}-0.00066 \\
(-0.17)\end{array}$ & $\begin{array}{c}-0.000535 \\
(-0.13)\end{array}$ & $\begin{array}{c}-0.00205 \\
(-0.49)\end{array}$ & $\begin{array}{c}-0.00209 \\
(-0.53)\end{array}$ & $\begin{array}{c}-0.00141 \\
(-0.33)\end{array}$ \\
\hline Studio & Controlled & Controlled & Controlled & Controlled & Controlled & Controlled \\
\hline Constant & $\begin{array}{c}-0.00762 \\
(-0.71)\end{array}$ & $\begin{array}{c}-0.0373^{* *} \\
(-2.63)\end{array}$ & $\begin{array}{c}-0.00938 \\
(-0.62)\end{array}$ & $\begin{array}{c}-0.000525 \\
(-0.06)\end{array}$ & $\begin{array}{c}-0.0141 \\
(-1.35)\end{array}$ & $\begin{array}{c}-0.0195 \\
(-1.40)\end{array}$ \\
\hline Observations & 68 & 68 & 68 & 68 & 68 & 68 \\
\hline $\begin{array}{c}\text { Adjusted } \\
R^{2}\end{array}$ & 0.344 & 0.375 & 0.31 & 0.33 & 0.389 & 0.311 \\
\hline
\end{tabular}

\section{The machine learning method}

The average experiment results of accuracy and F1-Score are shown in Table 4.11 and Figure 4.4. These findings are very similar to those derived in the previous case study, and their comparison confirmed that classical machine learning also outperformed MLR in the prediction of the stock price movement. The average accuracy of no more than $60 \%$ of hit ratios is usually reported as stock forecast result [46]; these prediction processes are also directly related to the corpus. In this case, while the review corpus was indirectly associated with the corresponding studio stock, this prediction results specifically yielded satisfactory results; this illustrates the effective learning process of machine learning.

Furthermore, most of the previous works [199] only utilized a few movie studios within a short period time. The advantage of this work, however, is that it covered Hollywood listed studios over a long period (two years). For some studios' stocks, there was a high-level average accuracy of $68.01 \%$ for Columbia by LR and $62.97 \%$ for Lionsgate by GNB. It also observed poor results for Universal, which is $41.62 \%$ by SVM and $43.89 \%$ by RF. This study 
Chapter 4. Results of the research

Table 4.10: The coefficient between UGC and box office (c).

\begin{tabular}{|c|c|c|c|c|c|c|}
\hline & (1) & (2) & (3) & (4) & (5) & (6) \\
\hline Variables & $\ln ($ Return3c & ) $\ln ($ Return3 & $\ln ($ Return30 & ) $\ln ($ Return 3 & ) $\ln ($ Return3 & ) $\ln ($ Return30 \\
\hline $\ln$ (pre_like1) & $\begin{array}{c}0.00174 \\
(0.95)\end{array}$ & & & & & \\
\hline $\ln ($ pre_like7) & & $\begin{array}{c}0.00428^{* *} \\
(2.13)\end{array}$ & & & & \\
\hline $\ln ($ pre_like30) & & & $\begin{array}{c}0.00269 \\
(1.28)\end{array}$ & & & \\
\hline $\ln ($ pre_share 1$)$ & & & & $\begin{array}{c}0.000907 \\
(0.49)\end{array}$ & & \\
\hline $\ln ($ pre_share7) & & & & & $\begin{array}{c}0.00356^{*} \\
(1.87)\end{array}$ & \\
\hline $\ln ($ pre_share 30 & & & & & & $\begin{array}{c}0.00245 \\
(1.23)\end{array}$ \\
\hline re_sp30 & $\begin{array}{l}0.424 \\
(1.47)\end{array}$ & $\begin{array}{c}0.369 \\
(1.33)\end{array}$ & $\begin{array}{l}0.438 \\
(1.56)\end{array}$ & $\begin{array}{c}0.462 \\
(1.62)\end{array}$ & $\begin{array}{l}0.431 \\
(1.56)\end{array}$ & $\begin{array}{c}0.469 \\
(1.67)\end{array}$ \\
\hline Friday & $\begin{array}{c}-0.00187 \\
(-0.24)\end{array}$ & $\begin{array}{c}-0.00168 \\
(-0.22)\end{array}$ & $\begin{array}{c}-0.00174 \\
(-0.22)\end{array}$ & $\begin{array}{c}-0.00196 \\
(-0.24)\end{array}$ & $\begin{array}{c}-0.00332 \\
(-0.43)\end{array}$ & $\begin{array}{c}-0.00296 \\
(-0.37)\end{array}$ \\
\hline Studio & Controlled & Controlled & Controlled & Controlled & Controlled & Controlled \\
\hline Constant & $\begin{array}{c}0.0022 \\
(0.11)\end{array}$ & $\begin{array}{c}-0.0474^{*} \\
(-1.75)\end{array}$ & $\begin{array}{c}-0.00022 \\
(-0.51)\end{array}$ & $\begin{array}{c}0.0117 \\
(0.66)\end{array}$ & $\begin{array}{c}-0.0126 \\
(-0.62)\end{array}$ & $\begin{array}{c}-0.0234 \\
(-0.90)\end{array}$ \\
\hline Observations & 68 & 68 & 68 & 68 & 68 & 68 \\
\hline $\begin{array}{c}\text { Adjusted } \\
R^{2}\end{array}$ & 0.286 & 0.327 & 0.295 & 0.278 & 0.316 & 0.293 \\
\hline
\end{tabular}

Table 4.11: The prediction accuracy and F1-Score of stock price movement.

\begin{tabular}{|c|c|c|c|c|c|c|c|}
\hline \multirow{2}{*}{$\begin{array}{l}\text { Studios } \\
\text { Studio }\end{array}$} & \multirow{2}{*}{$\begin{array}{c}\text { Baseline } \\
(\%)\end{array}$} & \multicolumn{6}{|c|}{ Classical machine learning $(\%)$} \\
\hline & & LR & $\mathrm{RF}$ & $\mathrm{XGB}$ & GNB & SVM & Average \\
\hline & \multicolumn{7}{|c|}{ Accuracy/F1Accuracy/F1Accuracy/F1Accuracy/F1Accuracy/F1Accuracy/F1Accuracy/F } \\
\hline & Score & Score & Score & Score & Score & Score & Score \\
\hline Disney & $52.91 / 54.03$ & $83.65 / 83.70$ & $75.61 / 76.67$ & $72.59 / 80.13$ & $68.19 / 66.87$ & $83.21 / 83.80$ & $72.69 / 74.20$ \\
\hline Fox & $59.31 / 58.94$ & $85.94 / 82.67$ & $79.12 / 77.16$ & $74.99 / 84.03$ & $73.19 / 70.15$ & $86.19 / 82.92$ & $76.46 / 75.98$ \\
\hline Lionsgate & $83.97 / 83.70$ & $83.65 / 80.06$ & $77.85 / 76.01$ & $73.53 / 83.31$ & $73.48 / 69.97$ & $81.19 / 80.55$ & $78.95 / 78.94$ \\
\hline Paramount & $50.04 / 53.03$ & $81.72 / 80.24$ & $73.09 / 73.50$ & $71.27 / 80.37$ & $69.79 / 70.38$ & $80.63 / 79.77$ & $71.09 / 72.89$ \\
\hline Columbia & $58.48 / 61.12$ & $79.67 / 81.83$ & $74.72 / 73.99$ & $70.97 / 81.10$ & $70.48 / 74.05$ & $80.05 / 83.11$ & $72.40 / 75.87$ \\
\hline Universal & $56.97 / 60.52$ & $83.60 / 85.51$ & $78.67 / 80.96$ & $78.52 / 86.84$ & $70.83 / 73.32$ & $84.82 / 85.65$ & $75.57 / 78.80$ \\
\hline Warner & $57.21 / 57.34$ & $83.94 / 82.84$ & $79.34 / 77.10$ & $77.34 / 85.94$ & $71.57 / 69.65$ & $84.11 / 83.52$ & $75.59 / 76.07$ \\
\hline
\end{tabular}


adopted audience review to directly predict the stock return of the studios and these forecast results benefit from unstructured data. Therefore, this work needs to analyze further the various factors of stocks through classical machine learning, which supports the marketing strategy of UGC for the studios.

These experimental results also fully demonstrate that a combined approach effectively solves the problem of structured and unstructured data in UGC. These ways can help studios make better use of UGC in social media and increase their profits. In particular, the application of machine learning based on the audience's rating can be extended to other social media platforms.

Compared with five algorithms in machine learning in two cases, these results fully illustrate the superiority of machine learning. Especially the classification performance of SVM, which is $0.002 \mathrm{~s}$ in time expend. However, both XGB and GNB are ensemble learning, their time expend are 0.044s. Ensemble learning takes significantly longer than machine learning, which reveals that training time consumes more time and susceptible to interference from noisy data.

\subsection{Application scenario 3}

This application scenario is divided into two case studies, which is mainly different in company performance. First case is audience sentiment classification, other case is stock market of the studios.

\subsubsection{Case study in audience sentiment classification}

This section reports that the result of accuracy evaluation in the audience sentiment in Table 4.12. Additionally, this section also computes the average of seven studios for convenient comparison in each algorithm. The model as HCAN achieved the best result that is $88.70 \%$ average accuracy in the eight algorithms. It observes that the word embedding with the pre-trained Glove is superior to the feature extraction by TF-IDF. The bag-of-word cannot capture semantic information on audience review, and when the word vocabulary is 
Table 4.12: Holistic comparison of traditional machine learning and deep learning for audience sentiment classification, the performance is measured based on accuracy, these values are a percentage i.e., the higher the better.

\begin{tabular}{cccccccccc}
\hline \multicolumn{9}{c}{ Algorithms } & \multicolumn{7}{c}{ Studios } \\
\hline & & Disney & Fox & Lionsgate & Paramount & Columbia & Universal & Warner & Average \\
\hline \multirow{2}{*}{ Traditional } & SVM & 73.70 & 74.33 & 72.08 & 68.33 & 74.29 & 72.93 & 75.29 & 72.99 \\
machine & DT & 68.15 & 69.26 & 64.78 & 64.32 & 69.55 & 66.05 & 68.78 & 67.27 \\
learning & LR & 79.08 & 82.00 & 70.07 & 67.35 & 81.46 & 78.44 & 79.06 & 76.78 \\
& RF & 72.34 & 76.34 & 69.16 & 65.96 & 74.15 & 72.66 & 73.50 & 72.02 \\
& AB & 73.50 & 76.66 & 71.53 & 68.83 & 76.86 & 76.58 & 74.97 & 74.13 \\
& GB & 71.79 & 77.22 & 70.07 & 65.38 & 78.48 & 75.83 & 74.73 & 73.36 \\
\hline Deep & CNN & 77.39 & 79.75 & 76.09 & 71.45 & 78.08 & 77.89 & 78.22 & 76.98 \\
learning & LSTM & 77.88 & 80.76 & 73.18 & 72.03 & 81.33 & 77.27 & 77.93 & 77.20 \\
\hline Attention & HTAN & 85.57 & 84.29 & 89.12 & 84.23 & 86.71 & 89.42 & 86.34 & 86.53 \\
mechanism & HCAN & 88.63 & 85.21 & 88.63 & 87.12 & 89.68 & 89.76 & 91.88 & 88.70 \\
\hline
\end{tabular}

too large, it encounters a limitation of the dimensional problem. In fact, word embedding by Glove is a kind of distributed feature representation. Since no pre-trained word embedding is under the possibility of overfitting, it should choose word training by Glove. The capability of word embedding to represent textual information is more reliable than using a word-of-bag approach.

Compared to among in traditional machine learning, deep learning with attention mechanism achieve an average of almost $18 \%$ and $15 \%$ improvement, respectively. One of the main reasons may be the introduction of a caution mechanism that highlights the essential parts of the review information. In addition, in the CNN and LSTM algorithms, they contain implicit sequence information, which is strongly focused on parts that are similar to word embedding. It ignores the sentiment features in the long-distance words, which leads to less improvement in deep learning. The proposed model HCAN achieves the most advanced performance on this data set. It is worth noting that HCAN performed $2.17 \%$ better than HTAN, which indicates limited capability in character level attention. This study believe this is because the importance of words depends not only on semantics but also on context and word order information. Extracting the features by the convolutional layer is more efficient than using only the character mechanism. The model also considers the word features order and their relevance to sentiment words in the review. The improvements prove that the model calculates attention weight of word from a global perspective by considering review information 
Table 4.13: Holistic comparison of traditional machine learning and deep learning for stock price movement, the performance is measured based on accuracy, these values are a percentage. i.e., the higher the better.

\begin{tabular}{cccccccccc}
\hline \multicolumn{3}{c}{ Algorithms } & \multicolumn{7}{c}{ Studios } \\
\hline & & Disney & Fox & Lionsgate & Paramount & Columbia & Universal & Warner & Average \\
\hline \multirow{2}{*}{ Traditional } & SVM & 61.05 & 66.26 & 63.81 & 55.97 & 58.91 & 55.96 & 56.84 & 59.83 \\
machine & DT & 61.72 & 63.90 & 61.98 & 55.65 & 59.91 & 63.16 & 53.26 & 59.94 \\
learning & LR & 50.50 & 57.65 & 63.89 & 50.66 & 67.46 & 71.26 & 62.63 & 60.58 \\
& RF & 66.15 & 64.04 & 64.42 & 61.07 & 65.05 & 62.07 & 53.54 & 62.33 \\
& AB & 60.01 & 59.94 & 66.08 & 48.51 & 51.24 & 67.8 & 69.02 & 59.94 \\
& GB & 58.17 & 71.05 & 71.10 & 50.65 & 68.52 & 55.57 & 67.04 & 63.16 \\
\hline Deep & CNN & 58.67 & 66.89 & 53.7 & 53.59 & 53.79 & 65.54 & 72.8 & 60.72 \\
learning & LSTM & 53.15 & 67.12 & 65.52 & 59.16 & 63.2 & 59.5 & 60.36 & 61.16 \\
\hline Attention & HTAN & 58.24 & 67.51 & 58.69 & 61.32 & 66.59 & 69.81 & 62.94 & 63.59 \\
mechanism & HCAN & 71.15 & 70.03 & 60.58 & 67.10 & 78.68 & 60.02 & 64.45 & 67.43 \\
\hline
\end{tabular}

and incorporates important word vectors into the complete review information. Thus, these results illustrate excellent performance through deep learning with the attention mechanism. The attention mechanism model facilitates the classification of the sentiment polarity and further determines the emotional orientation of eWOM.

\subsubsection{Case study in stock price movement}

Evaluation stock price movement in Table 4.13, these results have achieved similar to the former aspect. Average $67.43 \%$ for HCAN is the best result in these algorithms. HTAN obtains character-level embedding based on LSTM encoder in the context and computes embedding the weight between character level and word level. HCAN adopts a convolutional layer for the extraction feature strategy, which replaces the character level attention mechanism. Using bi-GRU encodes to guide the word level attention mechanism, each attention vector input corresponds to different semantics and emphasizes important words for the stock price movement. Such as crazy, funny, wonderful, etc. Therefore, the attention mechanism reflects the fine-grained emotional characteristics in the audience's review, which further promotes the positive growth of the firm's stock.

The impact of the attention mechanism in stock price movement. The word-level attention mechanism can capture the characters. The word-level attention mechanism mainly identifies different fine-grain features in audience review and defines the value of each review on the 
sentiment of eWOM. The word-level attention mechanism with the feature extracted by convolutional layers improves model performance compared to models that use only characterlevel or word-level attention mechanisms. This finding demonstrates that review features are reflected in different semantic levels, which presents the impact of audience review on eWOM at different levels, and further illustrates the effect of eWOM on the companies' performance.

Compared deep learning and attention mechanism, it finds that the attention mechanism takes more time than deep learning. Because of the complexity of the attention mechanism model, and the structure includes the CNN layer and the LSTM layer. This study sets the same number of epochs (Here is 20), the iteration time of the main attention mechanism is also longer than deep learning. This study selects 8, 16 and 32 for batch size; it finds that 16 fit the attention mechanism, which can update parameters multiple times in one epoch to accelerate convergence. The understanding in HCAN is that the two stacked attention units implemented provide richer identification capabilities not only at character level but also at word level. By combining the two a more nonlinear and integrative understanding is captured feature from audience review. The setup of these experiments also proves the superiority of the attention mechanism. 



\section{Chapter}

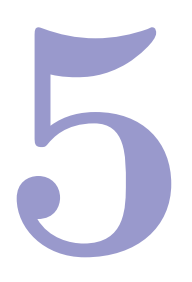

\section{Discussions and conclusions}

\subsection{Discussions}

This study investigates the application of AI technology in behavioral finance, that proposes a practical application framework for different application scenarios. The results show that the effective prediction for behavioral financial activities from human activity information, which provides decision support to company managers. The three application scenarios also prove the feasibility of the proposed application framework and can be extended to new application scenarios.

\subsubsection{AI technology in this study}

This study provides definitions, features and principles for AI technology. In particular, it utilizes traditional statistics, traditional machine learning and deep learning. This study also has developed different application frameworks in AI technology that presents the main findings and insights into the three application scenarios.

This study focuses on the introduction of NLP technology and machine learning as feature extraction, as presented in application scenario 1. With the recent development of deep learning and its application in NLP, language models have been greatly simplified. Deep learning models are usually based on LTSM and CNN. These two networks are generally 
jointly trained together. This study developed by combine models that model the event tuple by knowledge graph and deep learning. Besides, since the event tuple is very important, it designed the TransE model as a feature in the event tuple, thereby further improving semantic performance. Experimentally, the results prove that joint learning is the key to semantic feature extraction for stock market prediction.

This study also adopts traditional statistical models and machine learning for evaluating company performance, as presented in application scenario 2. As the powerful classification capability of machine learning, that can improve the accuracy of the results and can address the financial data be regarded as a typical structured data in the text. Many studies have turned to machine learning, which can handle unstructured data and structured data.

In predicting the accuracy of company stocks, this study observed that high precision does not mean better efficiency. Since it wants the model to bring more profit to the company, adjusting the model is more important than accuracy. Therefore, it will be interesting to introduce another type of attention mechanism based on deep learning. The attention mechanism is to optimize the network; for example, feature extraction is to strengthen the emotional characteristics of characters or words. In general, the performance of the attention mechanism will be better than deep learning. According to the function settings and network architecture, it chooses better parameters as in application scenario 3. For more complex model requirements, it may be necessary to introduce more complicated models (e.g., BERT [200 and transformer attention [112]) to obtain more features from the text data. In addition, compared to less complicated function settings, more complex function settings can significantly improve accuracy. Introducing the attention mechanism into deep learning harms less complicated feature settings because the attention mechanism requires higher-level data.

\subsubsection{AI technology development by extended data sources}

This study builds our three application fields based on the decision-making method. The leading architecture is using extended data resources, machine learning in extract features, feeding machine learning to create predictor models applied in finance. This architecture also can Expand to other fields. 
Currently, deep learning is the mainstream algorithm in AI technology. Most researchers prefer the deep learning model. Due to most of the extended data is time series data, which address by linear regression. However, its limitation is that it is difficult to deal with big data. In this case, LSTM is more suitable for such time series data and easy to address these problems. Different from LSTM, CNN can better handle the classification task, which is more ideal for the representation of static data. However, since most data changes over time, CNN is generally not a natural choice for time series data. Furthermore, innovatively converted onedimensional data into two-dimensional; these image data thus utilizes the unique features of CNN through adaptive filtering and pooling layer reduction [201]. Regardless of the extended data sources, many innovative methods seem to work well in a complex environment.

Previous study 202 shows that the hybrid machine learning model is preferable to a single model, which is to obtain better feature selection, as presented application scenarios 1 and 3. Researchers also try to configure network parameters to achieve higher performance. However, the interpretation of hybrid models can also be stressful. Through the experimental results of this study, it can be asserted that the performance of deep learning models is better than traditional machine learning. Deep learning also has the advantage of being able to process large amounts of data. As the continuous expansion of open source and application frameworks for deep learning libraries, the process of establishing and developing deep learning models is easier than ever. These improvements are most significant in deep learning-based algorithm implementation and NLP research.

In addition, graph convolutional networks (GCN) [203] has also attracted the attention of many scholars. GCN represents the nodes in the graph as a low-dimensional, real-valued, dense vector form so that the resulting vector form can have the ability to represent and reason in the vector space 203. However, GCN needs a higher quality in data quality, and the data needs to be converted into a graph [204]. Different models can be constructed through graph forms to provide researchers with opportunities to build financial applications with better performance. In short, text data integrated into the image data can improve the accuracy of the existing model, which also brings more application scenarios in the decision making method. 


\subsubsection{Implications for business research}

As far as financial activity applications are concerned, this research has touched on three application scenarios. In the foreseeable future, usually algorithmic trading, portfolio and risk assessment may continue to dominate the investigation of the financial field. Meanwhile, some new areas are beginning to receive more attention, not only because they represent relatively new research opportunities, but also because of their expected impact on the financial field [205], especially in portfolio management. Robo-advisor systems are emerging worldwide [206], which relies on high-performance automated decision support systems. Since AI technology is very suitable for this description, it is logically assumed that using AI technology will increase in the next few years. In this way, researchers can be very interested in the achievements that AI technology provides for the financial field. It may be necessary to integrate learning and optimization models to obtain a system with better performance.

The financial derivatives market is a research area that AI researchers usually avoid. Although there are many different products on the market, AI research is very lacking. For professionals working in the financial industry, these products provide incredible flexibility, from hedge funds to leveraged trading. Even if there are opportunities for AI researchers, because there is little research on financial derivatives, there is not much interest in this topic. In particular, selection strategy optimization, futures trading, option pricing, arbitrage trading and hedge fund benefit from the AI technology [207]. Furthermore, blockchain technology belongs to the technical application of these research fields [208]. Thus, it is worth exploring the infinite possibilities brought by these new technologies.

NLP technologies such as sentiment analysis and text mining have attracted researchers but have not been fully utilized. This study believes that the application of NLP to behavioral finance hides many opportunities. There is a lack of research work on behavioral finance published using AI technology. This phenomenon is mainly the difficulty in quantifying behavioral finance research through machine learning. However, new advances in the combination of NLP and machine learning can bring tremendous opportunities to business activities, even other fields. 


\subsubsection{Limitations}

Despite this research mentioned the contributions of each application scenario. However, this study still has some limitations since time and resources.

- Currently, data from human behavior information could predict business activity. However, this information has other types of data in human behavior, which is essential to collect the expression of different kinds of data, which better predict financial business activities. To achieve this goal, it is necessary to evaluate a variety of human behavioral data, which is also easy to collect more data.

- The benefits of these studies is to help the companies make decisions. However, The experimental setup in this study is quite straightforward and concise, and the stock price movement effect by many factors, experiment and observation data thereby needs a long period of accumulation in this study.

- This study only focuses on the application of AI technology in the financial sector. Correctly, in the implementation of behavioral finance, the proposed framework can be widely used in many other fields, such as economics and insurance, or more research may be involved in the future.

- The collection data in this study mainly crawls from the internet resource. However, the existence of these data may exist significant noise, and there are also privacy and security of data. This study can cooperate with the companies and obtain financial data, which needs to develop an effective method of data collection and sharing the results.

\subsubsection{Future work}

In this study, several findings have been examined through the analysis of different application scenarios. Nonetheless, some limitations remain to be addressed in the future. Thus, future work regarding new applications, new methods and new data sources needs to be done, the details are as follows: 
- Data sources. Financial news, stock data, structured and unstructured of data in UGC utilize in this study, these data have analyzed different behavioral financial scenarios. However, this research ignores other data sources or other platforms. For example, news disclosure or market reports, Twitter or Instagram. Moreover, UGC sources are only applicable to Hollywood movies in the United States, not including other countries or regions. Hence, it needs to expand the type of data and break geographical restrictions, which proposes the possibility of new data applications.

- Frameworks design. This study has adopted statistical models, traditional machine learning, deep learning and attention mechanisms in three application scenarios. However, many of the latest deep learning models have not been explored in this research. The new methods require us to expand the latest method in AI technology for new application scenarios, and address practical problems in different application scenarios.

- Application scenarios. Three application scenarios have enumerated in this research, which only contains the motion picture industry and financial investment. It can seek new application scenarios, such as prediction of tourism demands, energy economy, smart manufacturing, healthcare and environmental pollution, etc. It could greatly facilitate human life and promote the development of AI technology.

\subsection{Conclusions}

This research explores the application of AI technology in different scenarios of behavioral finance. An application framework is designed, which consists of data collection and result analysis. The feasibility of the application framework is verified through practical application scenarios. In this study, AI technology such as machine learning is used to analyze human behavioral information humans to improve prediction in business activity. In this process, the comparison between deep learning and the attention mechanism is compared in detail from a technical perspective.

In addition, this study also analyzed the financial situation of the seven countries in the European Union. We discussed machine learning to predict the financial distress of high-tech 
startups in various countries, especially the application for imbalanced data. This framework represents behavioral finance in new application scenarios. In summary, on the basis of the AI framework previously proposed, this study has summarized that artificial intelligence technology, and introduced modern architecture at the application of different fields, as shown in Figure 5.1. This also guides the future work in a new direction.

\subsubsection{Contributions}

This study aimed to apply advanced AI technology to behavioral finance scenarios. Under this goal, it proposed the AI framework for three application scenarios. Afterwards, three contributions can be summarized as the following:

1. Human behavior information was accurately extracted by NLP techniques, which helps to predict the stock market of the companies. In the anticipating stock market of renowned companies, it delivered an improved prediction of the stock market value for renowned companies in at least $3.6 \%$. Furthermore, in the stock market prediction of the studios, it presented the attention-based model for the stock market of the studios by $6.82 \%$ such improvement surpasses, those obtained using traditional machine learning and deep learning alone.

2. This research successfully explored UGC impacting on the studio performance, it revealed that opinion of costumers can be used, when UGC is properly collected to better estimate the economic performance of the involved companies. These effects have been reported previously for other markets, but in this research, the dimension in both audience sentiment and stock market of the studio was added, which enriches the feature extraction for the studio performance evaluation.

3. This study provides pioneering solutions for behavioral finance applications based on AI technology. In the anticipating stock market of renowned companies, the proposed knowledge graph method achieves better performance than that exhibited by an approach that uses only stock data, a bag-of-words method, and convolutional neural network. Moreover, in the studio performance evaluation, the experimental results de- 
termined that the proposed attention-based model is superior to both deep learning and traditional machine learning. 


\section{Bibliography}

[1] S. Hochreiter and J. Schmidhuber, "Long short-term memory," Neural computation, vol. 9, no. 8, pp. 1735-1780, 1997.

[2] K. Cho, B. Van Merriënboer, C. Gulcehre, D. Bahdanau, F. Bougares, H. Schwenk, and Y. Bengio, "Learning phrase representations using rnn encoder-decoder for statistical machine translation," arXiv preprint arXiv:1406.1078, 2014.

[3] K. Cho, A. Courville, and Y. Bengio, "Describing multimedia content using attentionbased encoder-decoder networks," IEEE Transactions on Multimedia, vol. 17, no. 11, pp. $1875-1886,2015$.

[4] A. Bordes, N. Usunier, A. Garcia-Duran, J. Weston, and O. Yakhnenko, "Translating embeddings for modeling multi-relational data," in Advances in neural information processing systems, 2013, pp. 2787-2795.

[5] "Facebook," https://www.facebook.com/DisneyZootopia/, accessed March, 2016.

[6] "Imdb," https://www.imdb.com/title/tt2948356/?ref_=nv_sr_srsg_0, accessed March, 2016.

[7] "Economy of europe," https://en.wikipedia.org/wiki/Economic_history_of_Europe, accessed January, 2020.

[8] "History of chinese currency," https://en.wikipedia.org/wiki/History_of_Chinese_ currency, accessed November, 2016.

[9] S. N. Neftci and M. Y. Menager-Xu, China's financial markets: an insider's guide to how the markets work. Elsevier, 2006. 
[10] A. Smith, "The wealth of nations [1776]," 1937.

[11] D. R. Fischel, "Use of modern finance theory in securities fraud cases involving actively traded securities," The Business Lawyer, pp. 1-20, 1982.

[12] H. Markowitz, "Portfolio selection," The journal of finance, vol. 7, no. 1, pp. 77-91, 1952.

[13] B. G. Malkiel and E. F. Fama, "Efficient capital markets: A review of theory and empirical work," The journal of Finance, vol. 25, no. 2, pp. 383-417, 1970.

[14] R. Thaler, "Toward a positive theory of consumer choice," Journal of Economic Behavior \& Organization, vol. 1, no. 1, pp. 39-60, 1980.

[15] W. F. De Bondt and R. Thaler, "Does the stock market overreact?" The Journal of finance, vol. 40, no. 3, pp. 793-805, 1985.

[16] D. I. Laibson, "Hyperbolic discounting and consumption," Ph.D. dissertation, Massachusetts Institute of Technology, 1994.

[17] N. Barberis and R. Thaler, "A survey of behavioral finance," Handbook of the Economics of Finance, vol. 1, pp. 1053-1128, 2003.

[18] G. M. Frankfurter and E. G. McGoun, "Market efficiency or behavioral finance: The nature of the debate," The Journal of Psychology and Financial Markets, vol. 1, no. 3-4, pp. 200-210, 2000.

[19] F. Hahn, "General equilibrium theory," The Public Interest, p. 123, 1980.

[20] R. C. Merton et al., "An intertemporal capital asset pricing model," Econometrica, vol. 41, no. 5, pp. 867-887, 1973.

[21] M. H. Miller, "The modigliani-miller propositions after thirty years," Journal of Economic perspectives, vol. 2, no. 4, pp. 99-120, 1988.

[22] J. D. MacBeth and L. J. Merville, "An empirical examination of the black-scholes call option pricing model," The journal of finance, vol. 34, no. 5, pp. 1173-1186, 1979. 
[23] R. Roll and S. A. Ross, "An empirical investigation of the arbitrage pricing theory," The Journal of Finance, vol. 35, no. 5, pp. 1073-1103, 1980.

[24] N. Woods, "The political economy of globalization," in The political economy of globalization. Springer, 2000, pp. 1-19.

[25] J. Begenau, M. Farboodi, and L. Veldkamp, "Big data in finance and the growth of large firms," Journal of Monetary Economics, vol. 97, pp. 71-87, 2018.

[26] A. M. Turing, "Computing machinery and intelligence," in Parsing the Turing Test. Springer, 2009, pp. 23-65.

[27] J. McCarthy, M. L. Minsky, N. Rochester, and C. E. Shannon, "A proposal for the dartmouth summer research project on artificial intelligence, august 31, 1955," AI magazine, vol. 27, no. 4, pp. 12-12, 2006.

[28] K. Schwab, The fourth industrial revolution. Currency, 2017.

[29] S. Russell and P. Norvig, "Intelligent agents," Artificial intelligence: A modern approach, vol. 74, pp. 46-47, 1995.

[30] D. Michie, D. J. Spiegelhalter, C. Taylor et al., "Machine learning," Neural and Statistical Classification, vol. 13, no. 1994, pp. 1-298, 1994.

[31] Y. LeCun, Y. Bengio, and G. Hinton, "Deep learning," nature, vol. 521, no. 7553, pp. 436-444, 2015.

[32] I. Goodfellow, Y. Bengio, and A. Courville, Deep learning. MIT press, 2016.

[33] R. R. Trippi and E. Turban, Neural networks in finance and investing: Using artificial intelligence to improve real world performance. McGraw-Hill, Inc., 1992.

[34] A. Botta, W. De Donato, V. Persico, and A. Pescapé, "Integration of cloud computing and internet of things: a survey," Future generation computer systems, vol. 56, pp. 684-700, 2016. 
[35] Z. Federico, "H10 charts that will change your perspective on artificial intelligence's growth," https://www.forbes.com/sites/louiscolumbus/2018/01/12/ 10-charts-that-will-change-your-perspective-on-artificial-intelligences-growth/ \#1919d7624758, accessed January, 2018.

[36] M. I. Jordan and T. M. Mitchell, "Machine learning: Trends, perspectives, and prospects," Science, vol. 349, no. 6245, pp. 255-260, 2015.

[37] X.-l. Zheng, M.-y. Zhu, Q.-b. Li, C.-c. Chen, and Y.-c. Tan, "Finbrain: when finance meets ai 2.0," Frontiers of Information Technology \&6 Electronic Engineering, vol. 20, no. 7, pp. 914-924, 2019.

[38] Y. Liu, Q. Zeng, H. Yang, and A. Carrio, "Stock price movement prediction from financial news with deep learning and knowledge graph embedding," in Pacific Rim Knowledge Acquisition Workshop. Springer, 2018, pp. 102-113.

[39] Y. Liu, Q. Zeng, J. Ordieres Meré, and H. Yang, "Anticipating stock market of the renowned companies: A knowledge graph approach," Complexity, vol. 2019, 2019.

[40] Y. Liu, H. Fei, Q. Zeng, B. Li, L. Ma, D. Ji, and J. Ordieres Meré, "Electronic word-ofmouth effects on studio performance leveraging attention-based model," Neural Computing and Applications, pp. 1-22, 2020.

[41] E. F. Fama, "Market efficiency, long-term returns, and behavioral finance," Journal of financial economics, vol. 49, no. 3, pp. 283-306, 1998.

[42] G. W. Schwert, "Why does stock market volatility change over time?" The journal of finance, vol. 44, no. 5, pp. 1115-1153, 1989.

[43] P. C. Tetlock, "Giving content to investor sentiment: The role of media in the stock market," The Journal of finance, vol. 62, no. 3, pp. 1139-1168, 2007.

[44] M. Baker and J. Wurgler, "Investor sentiment in the stock market," Journal of economic perspectives, vol. 21, no. 2, pp. 129-152, 2007. 
[45] J. Bollen, H. Mao, and X. Zeng, "Twitter mood predicts the stock market," Journal of computational science, vol. 2, no. 1, pp. 1-8, 2011.

[46] T. H. Nguyen, K. Shirai, and J. Velcin, "Sentiment analysis on social media for stock movement prediction," Expert Systems with Applications, vol. 42, no. 24, pp. 96039611, 2015.

[47] A. K. Nassirtoussi, S. Aghabozorgi, T. Y. Wah, and D. C. L. Ngo, "Text mining for market prediction: A systematic review," Expert Systems with Applications, vol. 41, no. 16, pp. 7653-7670, 2014.

[48] Q. Li, Y. Chen, J. Wang, Y. Chen, and H. Chen, "Web media and stock markets: A survey and future directions from a big data perspective," IEEE Transactions on Knowledge and Data Engineering, vol. 30, no. 2, pp. 381-399, 2017.

[49] F. Z. Xing, E. Cambria, and R. E. Welsch, "Natural language based financial forecasting: a survey," Artificial Intelligence Review, vol. 50, no. 1, pp. 49-73, 2018.

[50] M. Ahearne, C. B. Bhattacharya, and T. Gruen, "Antecedents and consequences of customer-company identification: Expanding the role of relationship marketing." Journal of applied psychology, vol. 90, no. 3, p. 574, 2005.

[51] H.-b. Kim, W. G. Kim, and J. A. An, "The effect of consumer-based brand equity on firms' financial performance," Journal of consumer marketing, 2003.

[52] S.-C. Chen, D. C. Yen, and M. I. Hwang, "Factors influencing the continuance intention to the usage of web 2.0: An empirical study," Computers in Human Behavior, vol. 28, no. 3, pp. 933-941, 2012.

[53] J. Krumm, N. Davies, and C. Narayanaswami, "User-generated content," IEEE Pervasive Computing, vol. 7, no. 4, pp. 10-11, 2008.

[54] T. W. Gruen, T. Osmonbekov, and A. J. Czaplewski, "ewom: The impact of customerto-customer online know-how exchange on customer value and loyalty," Journal of Business research, vol. 59, no. 4, pp. 449-456, 2006. 
[55] H. Baars and H.-G. Kemper, "Management support with structured and unstructured data - an integrated business intelligence framework," Information Systems Management, vol. 25, no. 2, pp. 132-148, 2008.

[56] B. Balducci and D. Marinova, "Unstructured data in marketing," Journal of the Academy of Marketing Science, vol. 46, no. 4, pp. 557-590, 2018.

[57] M. H. Jarrahi, "Artificial intelligence and the future of work: Human-ai symbiosis in organizational decision making," Business Horizons, vol. 61, no. 4, pp. 577-586, 2018.

[58] K.-Y. Goh, C.-S. Heng, and Z. Lin, "Social media brand community and consumer behavior: Quantifying the relative impact of user-and marketer-generated content," Information Systems Research, vol. 24, no. 1, pp. 88-107, 2013.

[59] V. Dhar and E. A. Chang, "Does chatter matter? the impact of user-generated content on music sales," Journal of Interactive Marketing, vol. 23, no. 4, pp. 300-307, 2009.

[60] S. Bikhchandani, D. Hirshleifer, and I. Welch, "A theory of fads, fashion, custom, and cultural change as informational cascades," Journal of political Economy, vol. 100, no. 5, pp. 992-1026, 1992.

[61] J. Liu and O. Toubia, "A semantic approach for estimating consumer content preferences from online search queries," Marketing Science, vol. 37, no. 6, pp. 930-952, 2018 .

[62] X. Luo, J. Zhang, and W. Duan, "Social media and firm equity value," Information Systems Research, vol. 24, no. 1, pp. 146-163, 2013.

[63] A. Colicev, A. Malshe, K. Pauwels, and P. O'Connor, "Improving consumer mindset metrics and shareholder value through social media: The different roles of owned and earned media," Journal of Marketing, vol. 82, no. 1, pp. 37-56, 2018.

[64] O. J. Rutz, G. P. Sonnier, and M. Trusov, "A new method to aid copy testing of paid search text advertisements," Journal of Marketing Research, vol. 54, no. 6, pp. 885-900, 2017. 
[65] O. J. Rutz and R. E. Bucklin, "From generic to branded: A model of spillover in paid search advertising," Journal of Marketing Research, vol. 48, no. 1, pp. 87-102, 2011.

[66] A. Timoshenko and J. R. Hauser, "Identifying customer needs from user-generated content," Marketing Science, vol. 38, no. 1, pp. 1-20, 2019.

[67] C. Dellarocas, N. Awad, and X. M. Zhang, "Using online reviews as a proxy of wordof-mouth for motion picture revenue forecasting," Available at SSRN 620821, 2004.

[68] Y. Liu, "Word of mouth for movies: Its dynamics and impact on box office revenue," Journal of marketing, vol. 70, no. 3, pp. 74-89, 2006.

[69] W. Duan, B. Gu, and A. B. Whinston, "The dynamics of online word-of-mouth and product sales - an empirical investigation of the movie industry," Journal of retailing, vol. 84, no. 2, pp. 233-242, 2008.

[70] A. Chakravarty, Y. Liu, and T. Mazumdar, "The differential effects of online wordof-mouth and critics' reviews on pre-release movie evaluation," Journal of Interactive Marketing, vol. 24, no. 3, pp. 185-197, 2010.

[71] A. E. Schlosser, "Can including pros and cons increase the helpfulness and persuasiveness of online reviews? the interactive effects of ratings and arguments," Journal of Consumer Psychology, vol. 21, no. 3, pp. 226-239, 2011.

[72] S. H. Kim, N. Park, and S. H. Park, "Exploring the effects of online word of mouth and expert reviews on theatrical movies' box office success," Journal of Media Economics, vol. 26 , no. 2 , pp. $98-114,2013$.

[73] R. Ullah, A. Zeb, and W. Kim, "The impact of emotions on the helpfulness of movie reviews," Journal of applied research and technology, vol. 13, no. 3, pp. 359-363, 2015.

[74] M. Hur, P. Kang, and S. Cho, "Box-office forecasting based on sentiments of movie reviews and independent subspace method," Information Sciences, vol. 372, pp. 608$624,2016$. 
[75] M. T. Lash and K. Zhao, "Early predictions of movie success: The who, what, and when of profitability," Journal of Management Information Systems, vol. 33, no. 3, pp. 874-903, 2016.

[76] J. H. Lee, S. H. Jung, and J. Park, "The role of entropy of review text sentiments on online wom and movie box office sales," Electronic Commerce Research and Applications, vol. 22, pp. 42-52, 2017.

[77] H. Baek, S. Oh, H.-D. Yang, and J. Ahn, "Electronic word-of-mouth, box office revenue and social media," Electronic Commerce Research and Applications, vol. 22, pp. 13-23, 2017.

[78] J. Xun and B. Guo, "Twitter as customer's ewom: an empirical study on their impact on firm financial performance," Internet Research, 2017.

[79] A. Mishra and S. Satish, "ewom: Extant research review and future research avenues," Vikalpa, vol. 41, no. 3, pp. 222-233, 2016.

[80] S. A. Vermeer, T. Araujo, S. F. Bernritter, and G. van Noort, "Seeing the wood for the trees: How machine learning can help firms in identifying relevant electronic word-ofmouth in social media," International Journal of Research in Marketing, vol. 36, no. 3, pp. 492-508, 2019.

[81] T. T. Kim, W. G. Kim, and H.-B. Kim, "The effects of perceived justice on recovery satisfaction, trust, word-of-mouth, and revisit intention in upscale hotels," Tourism management, vol. 30, no. 1, pp. 51-62, 2009.

[82] D. Yin, S. D. Bond, and H. Zhang, "Anxious or angry? effects of discrete emotions on the perceived helpfulness of online reviews," MIS quarterly, vol. 38, no. 2, pp. 539-560, 2014 .

[83] X. Luo, "Consumer negative voice and firm-idiosyncratic stock returns," Journal of marketing, vol. 71, no. 3, pp. 75-88, 2007. 
[84] X. Luo and J. Zhang, "How do consumer buzz and traffic in social media marketing predict the value of the firm?" Journal of Management Information Systems, vol. 30, no. 2, pp. 213-238, 2013.

[85] B. Wuthrich, V. Cho, S. Leung, D. Permunetilleke, K. Sankaran, and J. Zhang, "Daily stock market forecast from textual web data," in SMC'98 Conference Proceedings. 1998 IEEE International Conference on Systems, Man, and Cybernetics (Cat. No. 98CH36218), vol. 3. IEEE, 1998, pp. 2720-2725.

[86] G. P. C. Fung, J. X. Yu, and W. Lam, "Stock prediction: Integrating text mining approach using real-time news," in 2003 IEEE International Conference on Computational Intelligence for Financial Engineering, 2003. Proceedings. IEEE, 2003, pp. 395-402.

[87] W. Antweiler and M. Z. Frank, "Is all that talk just noise? the information content of internet stock message boards," The Journal of finance, vol. 59, no. 3, pp. 1259-1294, 2004 .

[88] M.-A. Mittermayer and G. F. Knolmayer, "Newscats: A news categorization and trading system," in Sixth International Conference on Data Mining (ICDM'06). Ieee, 2006, pp. 1002-1007.

[89] R. P. Schumaker and H. Chen, "Textual analysis of stock market prediction using breaking financial news: The azfin text system," ACM Transactions on Information Systems (TOIS), vol. 27, no. 2, pp. 1-19, 2009.

[90] S. S. Groth and J. Muntermann, "An intraday market risk management approach based on textual analysis," Decision Support Systems, vol. 50, no. 4, pp. 680-691, 2011.

[91] T. T. Vu, S. Chang, Q. T. Ha, and N. Collier, "An experiment in integrating sentiment features for tech stock prediction in twitter," in Proceedings of the workshop on information extraction and entity analytics on social media data, 2012, pp. 23-38.

[92] B. Wang, H. Huang, and X. Wang, "A novel text mining approach to financial time series forecasting," Neurocomputing, vol. 83, pp. 136-145, 2012. 
[93] Q. Li, T. Wang, P. Li, L. Liu, Q. Gong, and Y. Chen, "The effect of news and public mood on stock movements," Information Sciences, vol. 278, pp. 826-840, 2014.

[94] B. Weng, M. A. Ahmed, and F. M. Megahed, "Stock market one-day ahead movement prediction using disparate data sources," Expert Systems with Applications, vol. 79, pp. 153-163, 2017.

[95] F. Wolf, T. Poggio, and P. Sinha, "Human document classification using bags of words," 2006.

[96] K. Chen, P. Luo, L. Liu, and W. Zhang, "News, search and stock co-movement: Investigating information diffusion in the financial market," Electronic Commerce Research and Applications, vol. 28, pp. 159-171, 2018.

[97] X. Ding, Y. Zhang, T. Liu, and J. Duan, "Knowledge-driven event embedding for stock prediction," in Proceedings of coling 2016, the 26th international conference on computational linguistics: Technical papers, 2016, pp. 2133-2142.

[98] F. Z. Xing, E. Cambria, and Y. Zhang, "Sentiment-aware volatility forecasting," Knowledge-Based Systems, vol. 176, pp. 68-76, 2019.

[99] A. Picasso, S. Merello, Y. Ma, L. Oneto, and E. Cambria, "Technical analysis and sentiment embeddings for market trend prediction," Expert Systems with Applications, vol. 135, pp. 60-70, 2019.

[100] M. Kraus and S. Feuerriegel, "Decision support from financial disclosures with deep neural networks and transfer learning," Decision Support Systems, vol. 104, pp. 38-48, 2017.

[101] X. Ding, Y. Zhang, T. Liu, and J. Duan, "Deep learning for event-driven stock prediction," in Twenty-fourth international joint conference on artificial intelligence, 2015.

[102] E. Chong, C. Han, and F. C. Park, "Deep learning networks for stock market analysis and prediction: Methodology, data representations, and case studies," Expert Systems with Applications, vol. 83, pp. 187-205, 2017. 
[103] N. Terry, M. Butler, D. De'Armond et al., "The determinants of domestic box office performance in the motion picture industry," Southwestern Economic Review, vol. 32, pp. 137-148, 2011.

[104] R. W. Banz, "The relationship between return and market value of common stocks," Journal of financial economics, vol. 9, no. 1, pp. 3-18, 1981.

[105] B. R. Litman, "Predicting success of theatrical movies: An empirical study," The Journal of Popular Culture, vol. 16, no. 4, pp. 159-175, 1983.

[106] R. J. Calantone, N. Harmancioglu, and C. Droge, "Inconclusive innovation "returns": A meta-analysis of research on innovation in new product development," Journal of Product Innovation Management, vol. 27, no. 7, pp. 1065-1081, 2010.

[107] R. Sharda and D. Delen, "Predicting box-office success of motion pictures with neural networks," Expert Systems with Applications, vol. 30, no. 2, pp. 243-254, 2006.

[108] T. Kim, J. Hong, and P. Kang, "Box office forecasting using machine learning algorithms based on sns data," International Journal of Forecasting, vol. 31, no. 2, pp. 364-390, 2015.

[109] X. Liu, "Analyzing the impact of user-generated content on b2b firms' stock performance: Big data analysis with machine learning methods," Industrial marketing management, 2019.

[110] J. Du, H. Xu, and X. Huang, "Box office prediction based on microblog," Expert Systems with Applications, vol. 41, no. 4, pp. 1680-1689, 2014.

[111] B. M. Henrique, V. A. Sobreiro, and H. Kimura, "Literature review: Machine learning techniques applied to financial market prediction," Expert Systems with Applications, 2019 .

[112] A. Vaswani, N. Shazeer, N. Parmar, J. Uszkoreit, L. Jones, A. N. Gomez, E. Kaiser, and I. Polosukhin, "Attention is all you need," in Advances in neural information processing systems, 2017, pp. 5998-6008. 
[113] Y. Wang, M. Huang, L. Zhao et al., "Attention-based lstm for aspect-level sentiment classification," in Proceedings of the 2016 conference on empirical methods in natural language processing, 2016, pp. 606-615.

[114] H. Chen, M. Sun, C. Tu, Y. Lin, and Z. Liu, "Neural sentiment classification with user and product attention," in Proceedings of the 2016 Conference on Empirical Methods in Natural Language Processing, 2016, pp. 1650-1659.

[115] R. He, W. S. Lee, H. T. Ng, and D. Dahlmeier, "Exploiting document knowledge for aspect-level sentiment classification," arXiv preprint arXiv:1806.04346, 2018.

[116] J. Liu and Y. Zhang, "Attention modeling for targeted sentiment," in Proceedings of the 15th Conference of the European Chapter of the Association for Computational Linguistics: Volume 2, Short Papers, 2017, pp. 572-577.

[117] M. Song, H. Park, and K.-s. Shin, "Attention-based long short-term memory network using sentiment lexicon embedding for aspect-level sentiment analysis in korean," Information Processing \& Management, vol. 56, no. 3, pp. 637-653, 2019.

[118] Z. Hu, W. Liu, J. Bian, X. Liu, and T.-Y. Liu, "Listening to chaotic whispers: A deep learning framework for news-oriented stock trend prediction," in Proceedings of the Eleventh ACM International Conference on Web Search and Data Mining. ACM, 2018, pp. 261-269.

[119] Q. Liu, X. Cheng, S. Su, and S. Zhu, "Hierarchical complementary attention network for predicting stock price movements with news," in Proceedings of the 27th ACM International Conference on Information and Knowledge Management. ACM, 2018, pp. 1603-1606.

[120] W. Ling, I. Trancoso, C. Dyer, and A. W. Black, "Character-based neural machine translation," arXiv preprint arXiv:1511.04586, 2015.

[121] D. Golub and X. He, "Character-level question answering with attention," arXiv preprint arXiv:1604.00727, 2016. 
[122] Z. Yang, D. Yang, C. Dyer, X. He, A. Smola, and E. Hovy, "Hierarchical attention networks for document classification," in Proceedings of the 2016 conference of the North American chapter of the association for computational linguistics: human language technologies, 2016, pp. 1480-1489.

[123] E. Alpaydin, Introduction to machine learning. MIT press, 2014.

[124] T. O. Ayodele, "Types of machine learning algorithms," in New advances in machine learning. IntechOpen, 2010.

[125] R. Caruana and A. Niculescu-Mizil, "An empirical comparison of supervised learning algorithms," in Proceedings of the 23rd international conference on Machine learning, 2006, pp. 161-168.

[126] J. A. Suykens and J. Vandewalle, "Least squares support vector machine classifiers," Neural processing letters, vol. 9, no. 3, pp. 293-300, 1999.

[127] R. E. Wright, "Logistic regression." 1995.

[128] I. Rish et al., "An empirical study of the naive bayes classifier," in IJCAI 2001 workshop on empirical methods in artificial intelligence, vol. 3, no. 22, 2001, pp. 41-46.

[129] S. R. Safavian and D. Landgrebe, "A survey of decision tree classifier methodology," IEEE transactions on systems, man, and cybernetics, vol. 21, no. 3, pp. 660-674, 1991.

[130] L. Breiman, "Random forests," Machine learning, vol. 45, no. 1, pp. 5-32, 2001.

[131] Y. Freund, R. E. Schapire et al., "Experiments with a new boosting algorithm," in icml, vol. 96 . Citeseer, 1996, pp. 148-156.

[132] J. H. Friedman, "Greedy function approximation: a gradient boosting machine," Annals of statistics, pp. 1189-1232, 2001.

[133] — - "Stochastic gradient boosting," Computational statistics 8 data analysis, vol. 38, no. 4, pp. 367-378, 2002. 
[134] T. Chen, T. He, M. Benesty, V. Khotilovich, and Y. Tang, "Xgboost: extreme gradient boosting," R package version 0.4-2, pp. 1-4, 2015.

[135] G. Litjens, T. Kooi, B. E. Bejnordi, A. A. A. Setio, F. Ciompi, M. Ghafoorian, J. A. Van Der Laak, B. Van Ginneken, and C. I. Sánchez, "A survey on deep learning in medical image analysis," Medical image analysis, vol. 42, pp. 60-88, 2017.

[136] D. Stowell and M. D. Plumbley, "Automatic large-scale classification of bird sounds is strongly improved by unsupervised feature learning," PeerJ, vol. 2, p. e488, 2014.

[137] X. Zhang, J. Zhao, and Y. LeCun, "Character-level convolutional networks for text classification," in Advances in neural information processing systems, 2015, pp. 649657.

[138] A. Severyn and A. Moschitti, "Learning to rank short text pairs with convolutional deep neural networks," in Proceedings of the 38th international ACM SIGIR conference on research and development in information retrieval, 2015, pp. 373-382.

[139] J. Heaton, N. Polson, and J. H. Witte, "Deep learning for finance: deep portfolios," Applied Stochastic Models in Business and Industry, vol. 33, no. 1, pp. 3-12, 2017.

[140] A. Krizhevsky, I. Sutskever, and G. E. Hinton, "Imagenet classification with deep convolutional neural networks," in Advances in neural information processing systems, 2012, pp. 1097-1105.

[141] T. Mikolov, M. Karafiát, L. Burget, J. Černockỳ, and S. Khudanpur, "Recurrent neural network based language model," in Eleventh annual conference of the international speech communication association, 2010.

[142] J. C. Patra, R. N. Pal, B. Chatterji, and G. Panda, "Identification of nonlinear dynamic systems using functional link artificial neural networks," IEEE transactions on systems, man, and cybernetics, part b (cybernetics), vol. 29, no. 2, pp. 254-262, 1999.

[143] W. Shi, J. Caballero, F. Huszár, J. Totz, A. P. Aitken, R. Bishop, D. Rueckert, and Z. Wang, "Real-time single image and video super-resolution using an efficient sub- 
pixel convolutional neural network," in Proceedings of the IEEE conference on computer vision and pattern recognition, 2016, pp. 1874-1883.

[144] Y. Miao, M. Gowayyed, and F. Metze, "Eesen: End-to-end speech recognition using deep rnn models and wfst-based decoding," in 2015 IEEE Workshop on Automatic Speech Recognition and Understanding (ASRU). IEEE, 2015, pp. 167-174.

[145] T. Mikolov, S. Kombrink, L. Burget, J. Černockỳ, and S. Khudanpur, "Extensions of recurrent neural network language model," in 2011 IEEE international conference on acoustics, speech and signal processing (ICASSP). IEEE, 2011, pp. 5528-5531.

[146] M. Seo, A. Kembhavi, A. Farhadi, and H. Hajishirzi, "Bidirectional attention flow for machine comprehension," arXiv preprint arXiv:1611.01603, 2016.

[147] T. D. Sanger, "Optimal unsupervised learning in a single-layer linear feedforward neural network," Neural networks, vol. 2, no. 6, pp. 459-473, 1989.

[148] G. H. Dunteman, Principal components analysis. Sage, 1989, no. 69.

[149] A. Likas, N. Vlassis, and J. J. Verbeek, "The global k-means clustering algorithm," Pattern recognition, vol. 36, no. 2, pp. 451-461, 2003.

[150] X. Zhu and A. B. Goldberg, "Introduction to semi-supervised learning," Synthesis lectures on artificial intelligence and machine learning, vol. 3, no. 1, pp. 1-130, 2009.

[151] X. J. Zhu, "Semi-supervised learning literature survey," University of WisconsinMadison Department of Computer Sciences, Tech. Rep., 2005.

[152] L. P. Kaelbling, M. L. Littman, and A. W. Moore, "Reinforcement learning: A survey," Journal of artificial intelligence research, vol. 4, pp. 237-285, 1996.

[153] V. Mnih, K. Kavukcuoglu, D. Silver, A. A. Rusu, J. Veness, M. G. Bellemare, A. Graves, M. Riedmiller, A. K. Fidjeland, G. Ostrovski et al., "Human-level control through deep reinforcement learning," Nature, vol. 518, no. 7540, pp. 529-533, 2015.

[154] G. G. Chowdhury, "Natural language processing," Annual review of information science and technology, vol. 37, no. 1, pp. 51-89, 2003. 
[155] E. Cambria and B. White, "Jumping nlp curves: A review of natural language processing research," IEEE Computational intelligence magazine, vol. 9, no. 2, pp. 48-57, 2014 .

[156] T. Young, D. Hazarika, S. Poria, and E. Cambria, "Recent trends in deep learning based natural language processing," ieee Computational intelligenCe magazine, vol. 13, no. 3, pp. 55-75, 2018.

[157] Y. Goldberg, "Neural network methods for natural language processing," Synthesis Lectures on Human Language Technologies, vol. 10, no. 1, pp. 1-309, 2017.

[158] O. Levy and Y. Goldberg, "Neural word embedding as implicit matrix factorization," in Advances in neural information processing systems, 2014, pp. 2177-2185.

[159] Y. Goldberg and O. Levy, "word2vec explained: deriving mikolov et al.'s negativesampling word-embedding method," arXiv preprint arXiv:1402.3722, 2014.

[160] J. Pennington, R. Socher, and C. D. Manning, "Glove: Global vectors for word representation," in Proceedings of the 2014 conference on empirical methods in natural language processing (EMNLP), 2014, pp. 1532-1543.

[161] T. Mikolov, I. Sutskever, K. Chen, G. S. Corrado, and J. Dean, "Distributed representations of words and phrases and their compositionality," in Advances in neural information processing systems, 2013, pp. 3111-3119.

[162] W. Ling, C. Dyer, A. W. Black, and I. Trancoso, "Two/too simple adaptations of word2vec for syntax problems," in Proceedings of the 2015 Conference of the North American Chapter of the Association for Computational Linguistics: Human Language Technologies, 2015, pp. 1299-1304.

[163] B. Pang, L. Lee et al., "Opinion mining and sentiment analysis," Foundations and Trends® in Information Retrieval, vol. 2, no. 1-2, pp. 1-135, 2008.

[164] R. Feldman, "Techniques and applications for sentiment analysis," Communications of the $A C M$, vol. 56, no. 4, pp. 82-89, 2013. 
[165] B. Liu, "Sentiment analysis and opinion mining," Synthesis lectures on human language technologies, vol. 5, no. 1, pp. 1-167, 2012.

[166] M. Hu and B. Liu, "Mining and summarizing customer reviews," in Proceedings of the tenth ACM SIGKDD international conference on Knowledge discovery and data mining, 2004, pp. 168-177.

[167] E. Cambria, B. Schuller, Y. Xia, and C. Havasi, "New avenues in opinion mining and sentiment analysis," IEEE Intelligent systems, vol. 28, no. 2, pp. 15-21, 2013.

[168] A. Ortony, G. L. Clore, and A. Collins, The cognitive structure of emotions. Cambridge university press, 1990.

[169] P. D. Turney, "Thumbs up or thumbs down?: semantic orientation applied to unsupervised classification of reviews," in Proceedings of the 40th annual meeting on association for computational linguistics. Association for Computational Linguistics, 2002, pp. $417-424$.

[170] E. Cambria and A. Hussain, Sentic computing: Techniques, tools, and applications. Springer Science \& Business Media, 2012, vol. 2.

[171] H. Paulheim, "Knowledge graph refinement: A survey of approaches and evaluation methods," Semantic web, vol. 8, no. 3, pp. 489-508, 2017.

[172] Q. Wang, Z. Mao, B. Wang, and L. Guo, "Knowledge graph embedding: A survey of approaches and applications," IEEE Transactions on Knowledge and Data Engineering, vol. 29, no. 12, pp. 2724-2743, 2017.

[173] Z. Wang, J. Zhang, J. Feng, and Z. Chen, "Knowledge graph embedding by translating on hyperplanes," in Twenty-Eighth AAAI conference on artificial intelligence, 2014.

[174] G. Ji, K. Liu, S. He, and J. Zhao, "Knowledge graph completion with adaptive sparse transfer matrix," in Thirtieth AAAI conference on artificial intelligence, 2016.

[175] G. Ji, S. He, L. Xu, K. Liu, and J. Zhao, "Knowledge graph embedding via dynamic mapping matrix," in Proceedings of the 53rd Annual Meeting of the Association for 
Computational Linguistics and the 7th International Joint Conference on Natural Language Processing (Volume 1: Long Papers), 2015, pp. 687-696.

[176] G. K. Uyanık and N. Güler, "A study on multiple linear regression analysis," ProcediaSocial and Behavioral Sciences, vol. 106, no. 1, pp. 234-240, 2013.

[177] M. Stone and R. J. Brooks, "Continuum regression: cross-validated sequentially constructed prediction embracing ordinary least squares, partial least squares and principal components regression," Journal of the Royal Statistical Society: Series B (Methodological), vol. 52, no. 2, pp. 237-258, 1990.

[178] A. P. Dempster, M. Schatzoff, and N. Wermuth, "A simulation study of alternatives to ordinary least squares," Journal of the American Statistical Association, vol. 72, no. 357, pp. 77-91, 1977.

[179] C. Hsiao, "Panel data analysis - advantages and challenges," Test, vol. 16, no. 1, pp. $1-22,2007$.

[180] C. Goutte and E. Gaussier, "A probabilistic interpretation of precision, recall and f-score, with implication for evaluation," in European Conference on Information Retrieval. Springer, 2005, pp. 345-359.

[181] A. C. Cameron and P. K. Trivedi, Microeconometrics using stata. Stata press College Station, TX, 2009, vol. 5.

[182] T. E. Oliphant, "Python for scientific computing," Computing in Science 8 Engineering, vol. 9, no. 3, pp. 10-20, 2007.

[183] F. Pedregosa, G. Varoquaux, A. Gramfort, V. Michel, B. Thirion, O. Grisel, M. Blondel, P. Prettenhofer, R. Weiss, V. Dubourg et al., "Scikit-learn: Machine learning in python," Journal of machine learning research, vol. 12, no. Oct, pp. 2825-2830, 2011.

[184] A. Gulli and S. Pal, Deep learning with Keras. Packt Publishing Ltd, 2017. 
[185] M. Abadi, P. Barham, J. Chen, Z. Chen, A. Davis, J. Dean, M. Devin, S. Ghemawat, G. Irving, M. Isard et al., "Tensorflow: A system for large-scale machine learning," in 12th \{USENIX\} Symposium on Operating Systems Design and Implementation (\{OSDI\} 16), 2016, pp. 265-283.

[186] G. M. Marakas, Decision support systems in the 21st century. Prentice Hall Upper Saddle River, NJ, 2003, vol. 134.

[187] J. Rezaei, "Best-worst multi-criteria decision-making method," Omega, vol. 53, pp. 49-57, 2015.

[188] S. R. Axley, "Managerial and organizational communication in terms of the conduit metaphor," Academy of Management Review, vol. 9, no. 3, pp. 428-437, 1984.

[189] M. Jasemi, A. M. Kimiagari, and A. Memariani, "A modern neural network model to do stock market timing on the basis of the ancient investment technique of japanese candlestick," Expert Systems with Applications, vol. 38, no. 4, pp. 3884-3890, 2011.

[190] A. Fader, S. Soderland, and O. Etzioni, "Identifying relations for open information extraction," in Proceedings of the conference on empirical methods in natural language processing. Association for Computational Linguistics, 2011, pp. 1535-1545.

[191] X. Lin, Z. Yang, and Y. Song, "Intelligent stock trading system based on improved technical analysis and echo state network," Expert systems with Applications, vol. 38, no. 9, pp. $11347-11354,2011$.

[192] K.-j. Kim and I. Han, "Genetic algorithms approach to feature discretization in artificial neural networks for the prediction of stock price index," Expert systems with Applications, vol. 19, no. 2, pp. 125-132, 2000.

[193] J. Ramos et al., "Using tf-idf to determine word relevance in document queries," in Proceedings of the first instructional conference on machine learning, vol. 242. Piscataway, NJ, 2003, pp. 133-142.

[194] Y. Kim, "Convolutional neural networks for sentence classification," arXiv preprint arXiv:1408.5882, 2014. 
[195] J. Xu, K. Chen, X. Qiu, and X. Huang, "Knowledge graph representation with jointly structural and textual encoding," arXiv preprint arXiv:1611.08661, 2016.

[196] R. Xie, Z. Liu, J. Jia, H. Luan, and M. Sun, "Representation learning of knowledge graphs with entity descriptions," in Thirtieth AAAI Conference on Artificial Intelligence, 2016.

[197] F. Tian, B. Gao, E.-H. Chen, and T.-Y. Liu, "Learning better word embedding by asymmetric low-rank projection of knowledge graph," Journal of Computer Science and Technology, vol. 31, no. 3, pp. 624-634, 2016.

[198] N. C. Ellis, "Frequency effects in language processing: A review with implications for theories of implicit and explicit language acquisition," Studies in second language acquisition, vol. 24, no. 2, pp. 143-188, 2002.

[199] C. Ding, H. K. Cheng, Y. Duan, and Y. Jin, "The power of the "like" button: The impact of social media on box office," Decision Support Systems, vol. 94, pp. 77-84, 2017.

[200] J. Devlin, M.-W. Chang, K. Lee, and K. Toutanova, "Bert: Pre-training of deep bidirectional transformers for language understanding," arXiv preprint arXiv:1810.04805, 2018.

[201] M. Lin, Q. Chen, and S. Yan, "Network in network," arXiv preprint arXiv:1312.4400, 2013.

[202] P.-F. Pai and C.-S. Lin, "A hybrid arima and support vector machines model in stock price forecasting," Omega, vol. 33, no. 6, pp. 497-505, 2005.

[203] T. N. Kipf and M. Welling, "Semi-supervised classification with graph convolutional networks," arXiv preprint arXiv:1609.0290\%, 2016.

[204] J. Zhou, G. Cui, Z. Zhang, C. Yang, Z. Liu, L. Wang, C. Li, and M. Sun, "Graph neural networks: A review of methods and applications," arXiv preprint arXiv:1812.08434, 2018. 
[205] A. M. Ozbayoglu, M. U. Gudelek, and O. B. Sezer, "Deep learning for financial applications: A survey," arXiv preprint arXiv:2002.05786, 2020.

[206] T. Baker and B. Dellaert, "Regulating robo advice across the financial services industry," Iowa L. Rev., vol. 103, p. 713, 2017.

[207] G. J. Deboeck, Trading on the edge: neural, genetic, and fuzzy systems for chaotic financial markets. John Wiley \& Sons, 1994, vol. 39.

[208] Z. Zheng, S. Xie, H. Dai, X. Chen, and H. Wang, "An overview of blockchain technology: Architecture, consensus, and future trends," in 2017 IEEE international congress on big data (BigData congress). IEEE, 2017, pp. 557-564. 
